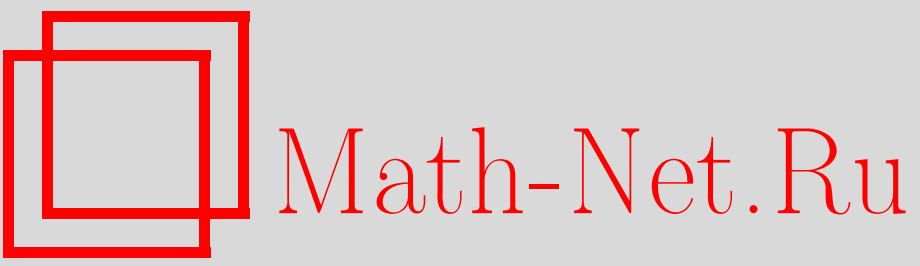

В. Г. Гельфрейх, В. Ф. Лазуткин, Расщепление сепаратрис: теория возмущений, экспоненциальная малость, УМН, 2001, том 56, выпуск 3, 79-142

DOI: https://doi.org/10.4213/rm394

Использование Общероссийского математического портала Math-Net.Ru подразумевает, что вы прочитали и согласны с пользовательским соглашением

http://www.mathnet.ru/rus/agreement

Параметры загрузки:

IP : 3.93 .64 .190

26 апреля 2023 г., 15:53:04 


\title{
РАСШЕПЛЕНИЕ СЕПАРАТРИС: ТЕОРИЯ ВОЗМУЩЕНИЙ, ЭКСПОНЕНЦИАЛЬНАЯ МАЛОСТЬ
}

\author{
В. Г. ГЕЛЬФРЕЙХ, В. Ф. ЛАЗУТКИН
}

\begin{abstract}
Настоящая статья содержит обзор основных резултатов, касающихся расщепления сепаратрис для отображений, сохраняющих площадь, и для гамильтоновых систем с полутора степенями свободы. Особое внимание уделено задачам, в которых расщепление сепаратрис оказьвается экспоненциально мальм по сравнению с параметром возмушения.

Библиография: 90 названий.
\end{abstract}

\section{СОДЕРЖАНИЕ}

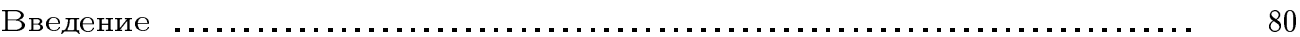

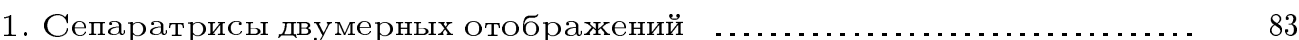

1.1. Параметризация сепаратрис .............................. 83

1.2. Нормальная форма сохраняющего площадь отображения в окрестности неподвижной гиперболической точки ........................... 85

1.3. Расщепление комплексных сепаратрис . . . . . . . . . . . . . . . . . . . . . . . 87

1.4. Несуществование интегралов движения, "хаос" ................... 88

2. Отображения, близкие к тождественному .......................... 91

2.1. Аппроксимация сепаратрис . ................................. 91

2.2. Существование двоякоасимптотических траекторий ............... 92

2.3. Величины, характеризующие расщепление сепаратрис ............ 94

2.4. Локальное выпрямление отображения ........................... 95

2.5. Экспоненциальная малость расщепления ... . . . . . . . . . . . . . . . . . . 96

3. Асимптотика для расщепления сепаратрис отображений ............ 98

3.1. Стандартное отображение ................................. 98

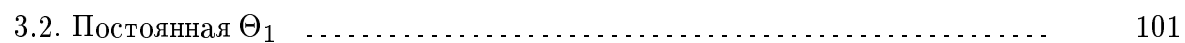

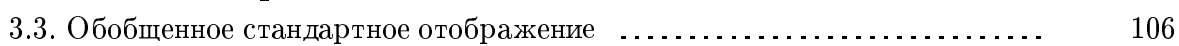

3.4. "Стохастическая паутина" ................................... 110

3.5. Расщепление сепаратрис около бифуркации ..................... 111

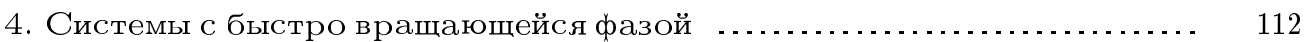

4.1. Точность разделения движений ............................. 113

4.2. Расщепление сепаратрис вблизи резонанса ..................... 115

4.3. Квалифицированная оценка расщепления ......................... 116

(С) В. Г. ГЕЛЬФРЕйх, В.Ф. ЛАЗУтКИн 2001 


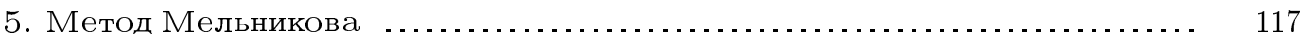

5.1. Функция Мельникова. Примеры ............................ 117

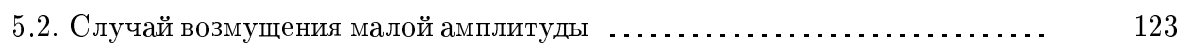

5.3. Маятник с большим высокочастотным возмущением . ............... 125

5.4. Схема доказательства ... . . . . . . . . . . . . . . . . . . . . . . . . . 130

5.5. Корректирующий множитель . . . . . . . . . . . . . . . . . . . . . . . . . . 134

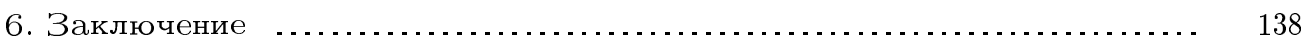

Список литературы . . . . . . . . . .

\section{Введение}

Рассмотрим гамильтонову систему с числом степеней свободы, большим единицы. Известно, что ее фазовое пространство может содержать два типа движений: регулярное и хаотическое. Регулярная компонента образована инвариантными торами, движение по которым является квазипериодическим. Эти торы являются объектом теории КАМ (Колмогоров-Арнольд-Мозер) [5], [50]. При достаточно общих условиях регулярная компонента имеет положительную меру Лебега. Для систем, близких к интегрируемьм, ее мера близка к полной мере фазового пространства. Регулярную компоненту можно считать хорошо изученной. В противоположность этому про хаотическую компоненту известно немного. Не очень понятно даже, что понимать под хаотической компонентой. Подходяшим определением является, по-видимому, следующее: хаотическая компонента - это инвариантное подмножество фазового пространства, состоящее из всех точек с ненулевыми показателями Ляпунова. Компютерные эксперименты заставляют верить тому, что если система обладает регулярной компонентой, то, как правило, хаотическая компонента также занимает множество положительной меры. В настоящее время доказательство положительности меры хаотической компоненты отсутствует. Если данное предположение справедливо, то к этому множеству применима теория Песина [68], которая, в частности, утверждает, что оно состоит из конечного или счетного числа компонент, на каждой из которых система в смысле теории меры изоморфна (в случае дискретного времени) произведению сдвига Бернулли на циклическую перестановку.

А. Пуанкаре, изучая задачу трех тел [70], обнаружил хаотическую компоненту и установил, что важную роль в ее образовании играют гиперболические инвариантные торы, гиперболические периодические траектории, а также их инвариантные (устойчивые и неустойчивые) многообразия. Напомним определение устойчивого, $W^{s}(\mathscr{T})$, и неустойчивого, $W^{u}(\mathscr{T})$, многообразий инвариантного подмножества $\mathscr{T}$ фазового пространства $X$ динамической системы (однопараметрической групшы преобразований) $f^{t}: X \rightarrow X$ :

$$
W^{s, u}(\mathscr{T})=\left\{p \in X: \lim _{t \rightarrow \pm \infty} \operatorname{dist}\left(f^{t}(p), \mathscr{T}\right)=0\right\}
$$

где знак “+" отвечает устойчивому, а "-" - неустойчивому многообразию. Мы предполагаем, что $X$ наделено расстоянием, которое мы обозначили символом dist. В ряде

В период работы над настоящей статьей авторы получали поддержку в виде следующих грантов: INTAS 97-0771, РФФИ 97-01-00612, РФФИ 97-01-00247, CRDF RM1-227. 
важных случаев $W^{u, s}(\mathscr{T})$ локально является гладким многообразием [67]. Для дифффеоморфизмов размерности два инвариантные многообразия одномерны, и их принято называть сепаратрисами.

Устойчивая сепаратриса не может пересекать ни саму себя, ни другую устойчивую сепаратрису. Однако могут сушествовать точки пересечения устойчивого и неустойчивого многообразий, назьваемые гомоклиническими или гетероклиническими в зависимости от того, соответствуют сепаратрисы одной периодической траектории (инвариантному тору) или нет. Траектории гомоклинических и гетероклинических точек А. Пуанкаре назьвал двоякоасимптотическими.

В интегрируемых системах сепаратрисы являются сдвоенными поверхностями: устойчивая сепаратриса одновременно является неустойчивой. Подобная ситуация является вырожденной. Согласно гамильтонову варианту теоремы Купки-Смейла [72] для систем общего положения все пересечения устойчивых и неустойчивых многообразий периодических траекторий трансверсальны. При этом сепаратрисы образуют запутанную сеть (см. рис. 1,2 ).

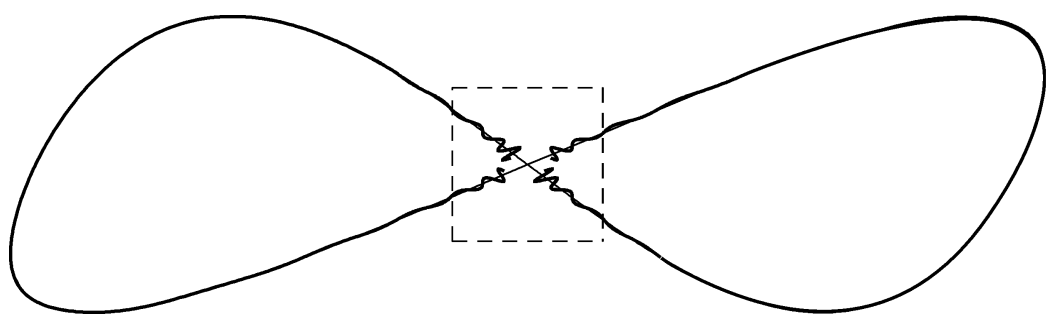

Рис. 1. Пример: расщепленные отрезки сепаратрис неподвижной гиперболической точки сохраняющего площадь отображения $x_{1}=x+y_{1}, y_{1}=y+\varepsilon x\left(1-x^{2}\right)$ при $\varepsilon=0.31$

Наличие трансверсальных двоякоасимптотических траекторий ведет к сложной динамике в близлежашей части фазового пространства, в том числе к существованию замкнутых инвариантных подмножеств, движение на которых носит "хаотический" характер и сопряжено со сдвигом Бернулли.

В аналитической теории возмущений расщепление сепаратрис часто оказывается экспоненщиально мальм по сравнению с естественньм параметром возмущения. Это явление впервые было обнаружено Пуанкаре при доказательстве расходимости формальных рядов теории возмущений в окрестности простого резонанса системы, близкой к интегрируемой [69; с. 732], [70; с. 406]. Подобная ситуация возникает во многих других задачах: расшепление сепаратрис под воздействием высокочастотных периодических по времени возмущений автономных систем; расшепление сепаратрис сохраняющих площадь отображений, близких к тождественному; расшепление сепаратрис в окрестности бифуркаций периодических траекторий в однопараметрических семействах сохраняющих площад отображений. Все эти задачи имеют между собой много общего. В частности, экспоненциальная малость расщепления для каждой из них может быть вьведена из теоремы Нейштадта [65].

В литературе по динамическим системам рассматриваются два типа гамильтоновых систем. Это потоки, описываемые системой дифференциальных уравнений Гамильтона, и динамические системы с дискретным временем, порожденные итерациями диффеоморфизмов, сохраняющих симплектическую структуру (в двумерном слу- 


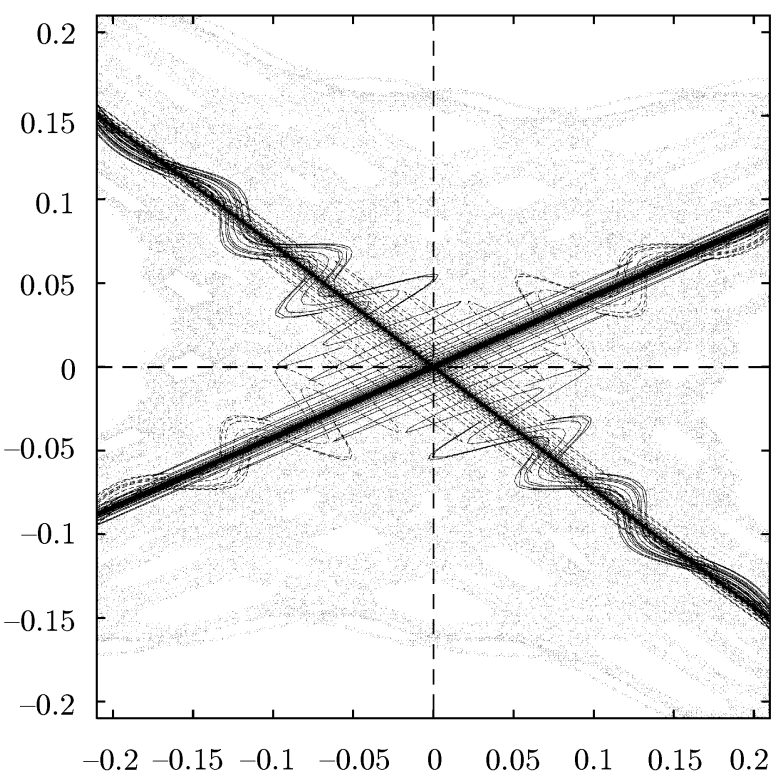

Рис. 2. Пример: расщепленные сепаратрисы на фоне, образованном итерациями одной точки. Показана часть фазового пространства, соответствующая квадрату на рис. 1

чае площадь). Связь между этими двумя типами может быть установлена с помошью конструкции, назьваемой сечением Пуанкаре [69], [50]. Переход от диффеоморфизмов к потокам осуществляется с помощью процедуры надстройки [50], [81]. Методы вычисления расщепления чувствительны к изменению вида уравнений. Поэтому теории для отображений и потоков в настоящее время развиваются параллельно.

Отметим особо, что аналитичность является ключевым свойством, определяющим масштабы рассматриваемых в настоящем обзоре явлений. Всегда, если только явно не указано обратное, отображения, многообразия и другие объекты будут подразумеваться аналитическими.

Оценки для расшепления сепаратрис, описьваемые в настояшем обзоре, получены путем выхода в комплексную область, причем, так сказать, с отходом от вешественной оси на большое расстояние, где у исследуемой системы имеется некоторая особенность. Общая идея состоит в том, что если в вещественной области интересующие нас эффекты экспоненциально малы и, в некотором смысле, “невидимы”, т.е. не могут быть обнаружены с помошью обычных средств, то возле особенности в комплексной области они становятся вполне заметными. Выявленные там эффекты удается продолжить назад в вешественную область, используя теоремы об аналитическом интеграле.

Опишем кратко содержание настоящего обзора. В первом разделе описьвается преимушественно динамика двумерных отображений в окрестности гиперболической неподвижной точки. Обсуждаются определение и основные свойства сепаратрис, а также связь расщепления сепаратрис с несуществованием аналитических интегралов движения. Несколько особо стоит теорема о расщеплении комплексных сепаратрис, устанавливающая, что в случае целого (комплексного) диффеоморфизма инвариант- 
ная кривая не может одновременно являться как устойчивой, так и неустойчивой сепаратрисой.

Второй раздел посвящен исследованию отображений, близких к тождественному. Приведены теоремы о близости их сепаратрис к сепаратрисам "предельных" потоков. Центральное место занимает теорема о возможности локально “вьпрямить” отображение с помошю аналитической замены координат. В качестве примера применения этой теоремы вьведена экспоненщиальная оценка сверху для расщепления сепаратрис.

В третьем разделе приведена коллекция асимптотических формул, описьваюших экспоненциальные расщепления сепаратрис для различных семейств отображений. Особое место в этой коллекции занимает стандартное отображение Чирикова, являющееся в некотором смысле эталонной задачей. Одним из первых общих результатов, касающихся оценок снизу для экспоненциально малого расщепления сепаратрис, является асимптотическая формула, описьвающая расщепление сепаратрис в окрестности гамильтоновой бифуркации рождения пары “седло-центр".

Раздел 4 посвящен методам исследования систем с полутора степенями свободы. Центральным результатом здесь является теорема Нейштадта о возможности разделения быстрых и медленных движений с точностью до экспоненциально малого остатка.

Раздел 5 посвящен применению популярного метода Мельникова к задачам об экспоненциально малом расщеплении сепаратрис. Приведены достаточные условия применимости этого метода. На примере маятника с высокочастотньм возмушением большой амплитуды проведен анализ границ применимости метода Мельникова. Проведен анализ асимптотических формул, получаюшихся за пределами этих границ.

\section{1. Сепаратрисы двумерных отображений}

1.1. Параметризация сепаратрис. Рассмотрим динамическую систему, порожденную итерациями диффеоморфизма $F: S \rightarrow S$ двумерной поверхности $S$. Точка $p_{0} \in S$ назьвается неподвижной точкой отображения $F$, если $F\left(p_{0}\right)=p_{0}$. Собственные числа $F^{\prime}\left(p_{0}\right)$ назьваются мультипликаторами.

Если на $S$ имеется форма площади $\Omega$, инвариантная относительно отображения $F$, то произведение мультипликаторов с необходимостью равняется единице. Пусть больший по модулю мультипликатор обозначен $\lambda$. Второй мультипликатор равен тогда $\lambda^{-1}$. Неподвижная точка сохраняющего площадь отображения может быть только одного из следующих типов:

а) гиперболическая, если $|\lambda|>1$;

б) параболическая (или вырожденная), если $\lambda= \pm 1$;

в) эллиптическая, если $|\lambda|=1$ и $\lambda \neq \pm 1$.

Отметим, что в определение "параболической" неподвижной точки иногда дополнительно включают условие невырожденности старшего нелинейного члена в разложении отображения в ряд Тейлора.

Для справедливости результатов, описанных в настоящем пункте, условие сохранения площади является излишним. Отметим, однако, что если отображение $F$ не сохраняет площадь, то единственное ограничение на возможные пары значений мультипликаторов возникает только из условия вешественности. Будем говорить, что не- 
подвижная точка является седловой, если один из мультипликаторов, $\lambda_{u}$, по модулю больше единицы, а другой, $\lambda_{s},-$ меньше. Гиперболическая неподвижная точка сохраняющего площадь отображения всегда седловая.

Согласно теореме Адамара-Перрона [67] седловая точка $p_{0}$ обладает одномерными локальными устойчивым и неустойчивым многообразиями:

$$
\begin{aligned}
& W_{\mathrm{loc}}^{s}\left(p_{0}\right)=\left\{p \in S: \operatorname{dist}\left(F^{n}(p), p_{0}\right)<\delta, \forall n \geqslant 0\right\} \\
& W_{\mathrm{loc}}^{u}\left(p_{0}\right)=\left\{p \in S: \operatorname{dist}\left(F^{n}(p), p_{0}\right)<\delta, \forall n \leqslant 0\right\},
\end{aligned}
$$

где $F^{n}=F \circ \cdots \circ F$ обозначает степень отображения $F$ относительно операции композиции, а $\delta$ - достаточно малое положительное число. Согласно устоявшейся традиции мы будем называть устойчивые и неустойчивые многообразия сепаратрисами. Касательная к сепаратрисе в точке $p_{0}$ является собственным вектором $F^{\prime}\left(p_{0}\right)$. Гиперболическая неподвижная точка и ее локальные сепаратрисы сохраняются при малых возмущениях и непрерывно (гладко, аналитически, ... ) зависят от отображения.

Локальные сепаратрисы могут быть неограниченно продолжены при помоши итераций диффеморфизма $F$. В результате получаются глобальные инвариантнье многообразия, которые можно определить как множество всех точек, стремящихся при итерациях $F$ (соответственно $\left.F^{-1}\right)$ к $p_{0}$ :

$$
\begin{aligned}
& W^{s}\left(p_{0}\right)=\left\{p \in S: \lim _{n \rightarrow+\infty} F^{n}(p)=p_{0}\right\}, \\
& W^{u}\left(p_{0}\right)=\left\{p \in S: \lim _{n \rightarrow-\infty} F^{n}(p)=p_{0}\right\} .
\end{aligned}
$$

Из определения очевидным образом следует, что неустойчивые сепаратрисы двух различных неподвижных точек не могут пересекаться.

Сепаратрисы являются одномерньми инвариантными кривыми, проходящими через точку $p_{0}$. Каждая сепаратриса является погружением без самопересечений вещественной прямой $\mathbb{R}$ в $S$. Удобно реализовать это вложение с помошью функции $\phi^{u}: \mathbb{R} \rightarrow S$, удовлетворяющей уравнению

$$
\phi^{u}\left(\lambda_{u} z\right)=F\left(\phi^{u}(z)\right), \quad \phi^{u}(0)=p_{0} .
$$

Отображение $\phi^{u}$ сопрягает сужение $F$ на $W^{u}$ с умножением на $\lambda_{u}$.

Все приведенные вьше определения и рассуждения легко переносятся на случай, когда $S$ является двумерньм комплексным многообразием. Если $F$ аналитично в окрестности $p_{0}$, то это уравнение имеет решение, аналитичное в окрестности нуля. В классе аналитических функций отображение $\phi^{u}$ определено с точностью до подстановки $z \mapsto C z$, где $C \neq 0$-произвольная постоянная.

Если отображение $F$ имеет целое аналитическое продолжение, то $\phi^{u}$ тоже является целой. Действительно, если $\phi^{u}$ аналитична в некотором круге $|z|<r$, то в силу уравнения (1.1) она аналитична также в большем круге $|z|<\left|\lambda_{u}\right| r$. Повторяя это рассуждение, продолжаем $\phi^{u}$ на всю комплексную плоскость.

Неподвижная точка разбивает каждую из сепаратрис на две части. Если соответствующий мультипликатор положителен, то каждая из половинок инвариантна относительно $F$. Положительности мультипликаторов можно достигнуть, рассмотрев 
вместо $F$ его вторую итерацию $F^{2}$. Сепаратрисы $F$ и $F^{2}$ очевидньм образом совпадают.

Часто бывает удобно произвести замену параметрана сепаратрисе, введя аддитивньй параметр $t=\ln z$ и положив

$$
\psi^{u}(t)=\phi^{u}\left(e^{t}\right)
$$

Функция $\psi=\psi^{u}$ удовлетворяет конечно-разностному уравнению

$$
\psi(t+h)=F(\psi(t))
$$

где $h=\ln \lambda_{u}$, снабженному граничным условием

$$
\lim _{t \rightarrow-\infty} \psi^{u}(t)=p_{0}
$$

Необходимо отметить, что последнее условие определяет аддитивную параметризацию неоднозначно. Вся неоднозначность заключена в возможности сделать замену параметра $t \mapsto t+a(t)$, где $a$ является произвольной функцией с периодом $h$.

Построение параметризации для устойчивой сепаратрисы полностью аналогично. В частности, можно повторить все приведенные выше рассуждения, заменив отображение $F$ на обратное. При этом удобно ввести параметр $t=-\ln z$, тогда параметризуюшая функция $\psi(t)=\psi^{s}(t)=\phi^{s}\left(e^{-t}\right)$ будет удовлетворять тому же уравнению (1.2) с $h=\ln \lambda_{s}^{-1}>0$, снабженному граничным условием

$$
\lim _{t \rightarrow+\infty} \psi^{s}(t)=p_{0}
$$

Точка называется периодической, если она является неподвижной точкой некоторой степени отображения $F$. Обобшение приведенных выше результатов на случай периодических точек прямолинейно.

Отметим, что в аналитическом случае теорему Адамара-Перрона можно доказать, иша решение уравнения (1.1) в виде рядов по степеням $z$ и строя для них сходяшиеся мажоранты.

Отметим, что для аналитических сохраняющих площадь отображений существование сепаратрис и свойства их параметризаций легко могут быть вьведены из теорем о нормальных формах, которые мы приведем в следующем пункте.

1.2. Нормальная форма сохраняющего площадь отображения в окрестности неподвижной гиперболической точки. Динамика в окрестности гиперболической неподвижной точки сохраняющего площадь диффеоморфизма $F$ локально устроена крайне просто: по теореме Гробмана-Хартмана движение топологически сопряжено с умножением на диагональную матрицу $\operatorname{diag}\left(\lambda, \lambda^{-1}\right)$.

Со времен Биркгофа известно, что формальной сохраняющей площадь заменой координат отображение $F$ можно привести к нормальной форме

$$
N:(\xi, \eta) \rightarrow(\xi \Lambda(\xi \eta), \eta / \Lambda(\xi \eta))
$$


где $\Lambda(E)=\lambda+\Lambda_{1} E+\Lambda_{2} E^{2}+\cdots$ является рядом по степеням произведения $E=\xi \eta$. Для доказательства этого факта можно построить замену координат в виде последовательности полиномиальных замен, приводящих отображение к нормальной форме с точностью до все более высоких степеней переменных $\xi$ и $\eta$.

Обычно нормальные формы Биркгофа расходятся. Однако Ю. Мозер [63] установил, что нормальная форма аналитична в случае гиперболической неподвижной точки сохраняюшего площадь отображения. Соответственно замена координат, приводяшая отображение к нормальной форме, также может быть выбрана аналитической.

ТеОРема 1.2.1 (Ю. Мозер). Существует симплектическая вещественно-аналитическая замена координат, приводящая сохраняющий площадь вещественно-аналитический диффеоморфизм $F$ в (комплексной) окрестности гиперболической неподвижной точки к нормальной форме Биркгофа (1.3).

В книге [77] имеется сравнительно несложное доказательство этого утверждения, основьваюшееся на построении сходяшихся мажорантных рядов для замены переменных и нормальной формы.

Нормальная форма определена единственным образом, а преобразование, приводящее отображение к нормальной форме, не единственно. Действительно, легко видеть, что нормальная форма инвариантна относительно замен координат вида

$$
\widetilde{\xi}=\xi \tau(\xi \eta), \quad \widetilde{\eta}=\eta / \tau(\xi \eta),
$$

где $\tau(\xi \eta)$ является сходящимся рядом по степеням произведения $\xi \eta$, при этом подразумевается $\tau(0) \neq 0$. Замена (1.4) сохраняет плошад, т.е. является симплектической. Этими заменами исчерпьвается весь произвол в выборе системы координат, приводящей отображение к нормальной форме. Кроме того, пусть $N$ и $\widetilde{N}$ являются аналитическими отображениями вида (1.3) с $|\Lambda(0)|=|\widetilde{\Lambda}(0)|=\lambda$. Если некоторое аналитическое сохраняющее площадь отображение $T$ сопрягает $N$ и $\widetilde{N}$, т.е. $T \widetilde{N}=N T$, то $T$ имеет вид (1.4), а $N=\widetilde{N}$. Из этого факта следует единственность нормальной формы.

Произведение координат $E=\xi \eta$ обладает интересньм свойством инвариантности: это аналитический интеграл движения, величина которого не зависит от выбора замены координат, приводящей отображение к нормальной форме. Действительно, замены вида (1.4) сохраняют произведение: $\xi \eta=\widetilde{\xi} \widetilde{\eta}$. Отметим, что в общем случае этот интеграл не имеет однозначного аналитического продолжения и, следовательно, не связан непосредственно с вопросом об интегрируемости отображения. Однако его сушествование означает, что в окрестности гиперболической неподвижной точки сохраняющее площадь отображение мохет быть локально вложено в автономный гамильтонов поток.

Если отображение $F=F_{\mu}$ зависит от параметра $\mu$, то при каждом фиксированном значении параметра оно может быть приведено к нормальной форме, которая, вообще говоря, тоже зависит от $\mu$. Если при некотором значении параметра мультипликаторы неподвижной точки обращаются в единицу, то замена координат, приводящая к нормальной форме, перестает быть определенной. Тем не менее, для следующего класса семейств были получены равномерные оценки на нормальную форму.

Для определенности поместим неподвижную точку в начало координат. Можно считать, что она теряет гиперболичность при $\mu=0$. Если при малых $\mu$ угол между 
собственньми векторами $F_{\mu}^{\prime}(0)$ отделен от нуля, то, выбирая их в качестве базисных, можно привести $F_{\mu}^{\prime}(0)$ к диагональному виду: $F_{\mu}^{\prime}(0)=A_{\mu}=\operatorname{diag}\left(\lambda, \lambda^{-1}\right)$. Пусть $\lambda=1+a \mu+o(\mu)$ для некоторого $a>0$. Кроме того, пусть нелинейная часть $F_{\mu}$ не слишком велика. Более точно, предположим, что отображение может быть записано в виде

$$
F_{\mu}(p)=A_{\mu} p+\mu f(p, \mu),
$$

где $f$ обозначает нелинейные члены разложения $F_{\mu}$ в ряд Тейлора: $f(0, \mu)=0$, $f^{\prime}(0, \mu)=0$.

Пусть $\mu_{0}>0$, а $U \subset \mathbb{C}^{2}$ является такой окрестностью начала координат, что отображение $F_{\mu}(p)$ аналитично при $p \in U$ и непрерывно на $U \times\left[0, \mu_{0}\right]$.

Теорема 1.2 .2 (Э. Фонтик, К. Симо [23], [22]). Существует такое положительное число $\mu_{1}, 0<\mu_{1}<\mu_{0}$, и такая (комплексная) окрестность начала координат $V \subset U$, что семейство $F_{\mu}: U \rightarrow \mathbb{C}^{2}$ сохраняющих площадь аналитических диффеоморфизмов вида (1.5) может быть при каждом $\mu \in\left(0, \mu_{1}\right)$ приведено к нормальной форме Биркгофа. При этом как нормальная форма, так и замена координат аналитичны на $V$ при фиксированном $\mu$ и ограничены на $V \times\left(0, \mu_{1}\right)$.

Доказательство теоремы основывается на построении не зависящих от $\mu$ мажорант для нормальной формы и замены координат.

1.3. Расщепление комплексных сепаратрис. Точки пересечения $W^{s}\left(p_{0}\right) \cap$ $W^{u}\left(p_{0}\right) \backslash\left\{p_{0}\right\}$ называются гомоклиническими. Если же пересекается устойчивая сепаратриса одной точки с неустойчивой сепаратрисой другой, то соответствуюшие точки называются гетероклиническими. В силу инвариантности сепаратрис все образы и прообразы гомоклинической (гетероклинической) точки являются гомоклиническими (гетероклиническими). Пуанкаре назьвал траектории гомоклинических и гетероклинических точек двоякоасимптотическими.

Пример линейного отображения $(x, y) \mapsto\left(\lambda x, \lambda^{-1} y\right)$ показывает, что сепаратрисы могут не иметь точек пересечения, отличных от гиперболической неподвижной точки.

Гомоклинические и гетероклинические точки могут быть точками трансверсального пересечения, точками касания конечного порядка или точками касания бесконечного порядка. Будем говорить, что сепаратрисы расщеплены, если их пересечение не содержит точек касания бесконечного порядка.

Teоpema 1.3.1 (Sh. Ushiki [87]). Пусть $S=S_{1} \times S_{2}$, где $S_{1}$ и $S_{2}$ - комплексные связные кривые, ни одна из которых не есть сфера Римана. Тогда все устойчивые и неустойчивые многообразия седловых неподвижных точек аналитического диффеморфизма $F: S \rightarrow S$ расщеплены.

Теорема приведена в формулировке, предложенной в работе [54]. Она показьвает, что для аналитического диффеоморфизма расшепление сепаратрис может быть обусловлено геометрией фазового пространства. Из теоремы, в частности, следует, что если отображение $F$ имеет аналитическое продолжения до диффеоморфизма $\mathbb{C}^{2} \rightarrow \mathbb{C}^{2}$, то все его гомоклинические и гетероклинические точки либо трансверсальны, либо являются точками касания конечного порядка. Однако теорема не дает никакой информации о четности порядка точек касания. 
Из теоремы, в частности, следует, что если гамильтонова система с одной степенњю свободы имеет двоякоасимптотическую траекторию, то отображение, сдвигающее точку вдоль ее траектории за единицу времени, не имеет целого аналитического продолжения до диффеоморфизма $\mathbb{C}^{2} \rightarrow \mathbb{C}^{2}$.

Отметим, что для справедливости теоремы не требуется, чтобы диффеоморфизм $F$ сохранял площадь.

Доказательство теоремы легко переносится на случай одномерных инвариантных многообразий аналитических автоморфизмов $n$-мерных многообразий вида $S=S_{1} \times$ $S_{2} \times \cdots \times S_{n}$

Из доказательства теоремы следует также, что если произведение мультипликаторов, отвечающих устойчивой и неустойчивой сепаратрисам, не равно единице, то эти сепаратрисы расшеплены независимо от условий на геометрию фазового пространства.

В качестве примера применения теоремы рассмотрим обобшенное стандартное отображение:

$$
(x, y) \mapsto\left(x+y+V^{\prime}(x), y+V^{\prime}(x)\right) .
$$

Если $V(x)$ является целой функцией, то сепаратрисы всех гиперболических периодических точек расщеплены. То же верно для отображения Эно

$$
(x, y) \mapsto\left(1-a x^{2}+y, b x\right) .
$$

Как показьвает следующий пример [80], [62], в случае, когда $V(x)$ не является целой функцией, стандартное отображение может быть интегрируемым, а его сепаратрисы - нерасшепленными. Пусть

$$
V^{\prime}(x, \varepsilon)=\varepsilon \frac{x-x^{3}}{1+\varepsilon x^{2} / 2}
$$

где $\varepsilon$ является положительным параметром. Соответствующее стандартное отображение имеет полиномиальньй интеграл движения

$$
H(x, y, \varepsilon)=\frac{y^{2}}{2}+\varepsilon\left(-\frac{x^{2}}{2}+\frac{x^{4}}{4}+\frac{x y}{2}-\frac{x^{3} y}{2}+\frac{x^{2} y^{2}}{4}\right) .
$$

Устойчивая и неустойчивая сепаратрисы гиперболической неподвижной точки $(0,0)$ совпадают. Они принадлежат линии уровня $H(x, y, \varepsilon)=0$, имеюшей вид "восьмерки", а все гомоклинические точки являются точками касания бесконечного порядка.

1.4. Несуществование интегралов движения, “хаос". Функция $H: S \rightarrow \mathbb{R}$ $(H: S \rightarrow \mathbb{C})$ называется интегралом движения, если она постоянна на траекториях динамической системы, т.е. имеет место равенство $H \circ F=H$. Постоянная функция всегда является интегралом движения. Будем назьвать диффеоморфизм $F$ интегрируемым, если он обладает непостоянным (аналитическим) интегралом движения.

Примером интегрируемого двумерного диффеморфизма является сдвиг за фиксированное время $t$ вдоль траекторий автономной гамильтоновой системы с одной степенњю свободы. Интегралом движения в этом случае является функция Гамильтона $H$. Говорят, что соответствуюшее отображение вложено в поток. 
Известно небольшое число явно заданных интегрируемых отображений. Одно из них описано в конце предыдущего пункта, ряд других примеров можно найти в работе Ю. Б. Суриса [80].

Динамика двумерных отображений, обладаюших нетривиальным интегралом движения, достаточно проста, так как траектории принадлежат линиям уровня интеграла.

Следующая теорема была известна еще А. Пуанкаре [69]. Приведенное ниже доказательство близко к [14].

Теорема 1.4.1 (А. Пуанкаре). Если вещественно-аналитический автоморфизм $F$ двумерного многообразия $S$ имеет гомоклиническую или гетероклиническую точку, не являюшуюся точкой касания бесконечного порядка, то он не обладает непостоянныц (однозначным) аналитическим интегралом движения.

ДОКАЗАТЕЛЬСТВО. Пусть $p_{h}$ является гомоклинической (или гетероклинической) точкой, $p_{h} \in W^{u}\left(p_{0}\right) \cap W^{s}\left(p_{1}\right)$. По теореме Гробмана-Хартмана отображение в окрестности гиперболической неподвижной точки топологически сопряжено с линейным. Обозначим через $V$ соответствуюшую окрестность точки $p_{1}$. Так как $p_{h}$ лежит на устойчивом многообразии, для достаточно большого $N$ можно считать, что $F^{n}\left(p_{h}\right)$ принадлежит $V$ при всех $n \geqslant N$. Рассмотрим маленький отрезок неустойчивой сепаратрисы $\ell$, проходящий через точку $F^{N}\left(p_{h}\right)$. При итерациях этот отрезок будет растягиваться и приближаться к локальной неустойчивой сепаратрисе (см. рис. 3). Напомним, что в вышеописанной координатной системе отображение действует как умножение на диагональную матрицу $\operatorname{diag}\left(\lambda_{u}, \lambda_{s}\right)$.

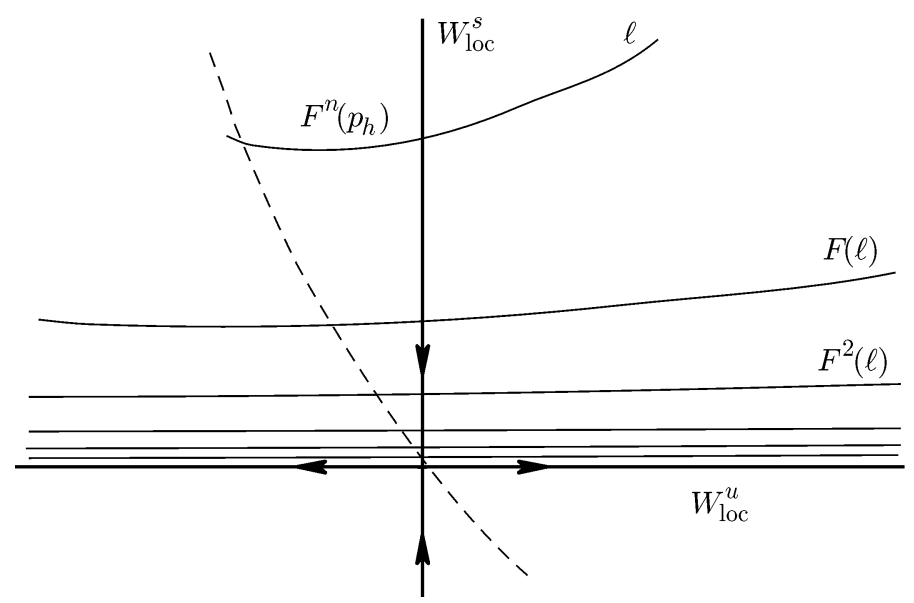

Р ис. 3. Иллюстрация к доказательству теоремы 1.4.1

Любая аналитическая кривая, лежашая в $V$ и трансверсально пересекающая локальную неустойчивую сепаратрису, имеет бесконечное число пересечений с образами $\ell$. 
Предположим, что диффеоморфизм $F$ обладает аналитическим интегралом движения $H$. Несложно видеть, что в силу непрерьвности

$$
H\left(p_{h}\right)=H\left(p_{0}\right)=H\left(p_{1}\right)=H\left(W^{u}\left(p_{0}\right)\right)=H\left(W^{s}\left(p_{1}\right)\right) .
$$

Без ограничения обшности можно считать это значение равньм нулю. Тогда сужение $H$ на аналитическую кривую является аналитической функцией одной переменной, имеюшей точку накопления нулей. Следовательно, интеграл тождественно равен нулю на всей этой кривой. В силу произвольности кривой интеграл равен нулю на $V$. По принципу аналитического продолжения интеграл равен нулю на всем многообразии $S$.

Параллельное утверждение для потоков вьглядит следуюшим образом. Пусть система с двумя степенями свободы и гамильтонианом $H$ имеет гиперболическую периодическую траекторию, принадлежашую вместе с соответствующими сепаратрисами уровню постоянной энергии $M_{c}=\{H=c\}$. Пусть хотя бы на одной сепаратрисе имеется гомоклиническая траектория, трансверсальная в $M_{c}$. Тогда система не имеет аналитического интеграла движения, независимого от $H$ в окрестности $M_{c}$.

Обобщение этого утверждения на случай более высокой размерности или на случай инвариантных многообразий, связанных с гиперболическими положениями равновесия, не столь прямолинейно, как могло бы показаться на первьй взгляд.

В качестве примера рассмотрим систему с гамильтонианом

$$
H=\frac{y_{1}^{2}}{2}+\cos x_{1}+x_{2} y_{2}
$$

Сепаратрисы неподвижной точки, расположенной в начале координат, являются произведениями сепаратрисы маятника на прямые $x_{2}=0$ и $y_{2}=0$ соответственно. Эти сепаратрисы пересекаются трансверсально внутри уровня постоянной энергии $H=1$. Однако система имеет очевидньй независимый интеграл движения $I=x_{2} y_{2}$. Более подробно связь расщепления сепаратрис и несушествования интегралов движения обсуждается в обзоре В.В. Козлова [45] и в книге того же автора [46]. Пример трансверсальной гомоклинической траектории в интегрируемой системе приведен в $[18]$.

Расщепление сепаратрис может приводить не только к несуществованию интегралов движения, но и к "хаотическому" поведению траекторий. Это находит выражение в нерегулярном поведении отдельных траекторий, а также в высокой чувствительности движения к изменению начальных условий. Основы общей теории были заложены в работе В.М. Алексеева [1]. Моделью такого движения является отображение, называемое подковой Смейла (см., например, книгу [59]).

Сепаратрисы седловых точек являются вложениями $\mathbb{R}$ в фазовое пространство. При этом сепаратрисы наследуют упорядоченность из $\mathbb{R}$. Следуюший факт был известен еше А. Пуанкаре [69].

Пусть диффеоморфизм $F: S \rightarrow S$, где $S$ является компактным подмножеством плоскости $\mathbb{R}^{2}$, сохраняет площадь. Между любыми двумя различными гомоклиническими точками на сепаратрисе имеется по крайней мере еще одна гомоклиническая точка. 
ДокаЗАТеЛьСтво. Пусть $p_{h}, q_{h} \in W^{u}\left(p_{0}\right) \cap W^{s}\left(p_{1}\right)$. Рассмотрим отрезки $\ell_{u}$ и $\ell_{s}$ устойчивой и неустойчивой сепаратрис с конщами в точках $p_{h}$ и $q_{h}$. Поведение этих отрезков при применении к ним отображения $F$ зависит от того, принадлежат этим отрезкам неподвижные точки или нет. Разберем последовательно соответствующие варианты и покажем, что в любом случае внутри отрезка $\ell_{u}$ имеется гомоклиническая (гетероклиническая) точка.

Если неподвижная точка принадлежит отрезку $\ell_{u}$, то на $\ell_{u}$ имеется бесконечно много гомоклинических точек, так как в этом случае $F^{-n}\left(p_{h}\right) \in \ell_{u}$ при всех $n \geqslant 0$.

Пусть отрезок $\ell_{u}$ не содержит неподвижной точки. Все точки пересечения $\ell_{u} \cap \ell_{s}$ являются гомоклиническими. Если отрезки имеют точку пересечения, отличную от $p_{h}$ и $q_{h}$, то она является искомой гомоклинической точкой.

Если же пересечение не содержит точек, отличных от конечных, то отрезки ограничивают фигуру П, имеюшую положительную площадь. При этом $F^{n}\left(\ell_{u}\right) \cap \ell_{u}=\varnothing$ при любом $n \neq 0$. Возможно, что неподвижная точка $p_{1} \in \ell_{s}$, в таком случае $F\left(\ell_{s}\right) \in \ell_{s}$ и отсутствие пересечений у $F\left(\ell_{u}\right)$ и $\ell_{s}$ противоречит сохранению плошади.

Пусть, наконец, $p_{1} \notin \ell_{s}$. Согласно теореме Пуанкаре о возврашении существует сколь угодно большое число итераций $n>0$, при котором $\operatorname{mes}\left(F^{n}(\Pi) \cap \Pi\right) \neq 0$. Множество $F^{n}(\Pi)$ ограничено отрезками $F^{n}\left(\ell_{u}\right)$ и $F^{n}\left(\ell_{s}\right)$. Так как $F^{n}\left(\ell_{u}\right) \cap \ell_{u}=\varnothing$ и $F^{n}\left(\ell_{s}\right) \cap \ell_{s}=\varnothing$ при достаточно большом $n$, то $F^{n}\left(\ell_{u}\right) \cap \ell_{s} \neq \varnothing$ либо $F^{n}\left(\ell_{s}\right) \cap \ell_{u} \neq \varnothing$.

Прообразы этих точек пересечения являются искомьми гомоклиническими точками.

Из приведенных рассуждений следует, что между любыми двумя гомоклиническими траекториями на сепаратрисе имеется бесконечно много других гомоклинических траекторий.

\section{2. Отображения, близкие к тождественному}

2.1. Аппроксимация сепаратрис. В настоящем разделе мы получим оценки для расщепления сепаратрис отображений вида

$$
F_{\varepsilon}(p)=p+\varepsilon f(p)+\varepsilon^{2} g(p, \varepsilon), \quad p \in \mathbb{R}^{2} .
$$

При малых $\varepsilon$ естественно сравнить это отображение с потоком, порождаемым дифференциальным уравнением

$$
\dot{p}=f(p) .
$$

Дифференциальное уравнение (2.2) назьвают предельным потоком отображения $F_{\varepsilon}$. Подобная связь между потоком и отображением возникает при численном интегрировании дифференциального уравнения (2.2). К виду (2.1) может быть приведено также отображение последования за период возмущения для высокочастотного периодического по времени возмущения уравнения (2.2).

Пусть система (2.2) имеет седловую точку $p_{0}$ с характеристическими показателями $\pm a \neq 0$, а также двоякоасимптотическую траекторию $p=\sigma_{0}(t)$. Тогда при всех достаточно малых $\varepsilon$ отображение (2.1) имеет гиперболическую неподвижную точку $p_{\varepsilon}=p_{0}+O(\varepsilon)$ с мультипликаторами $\lambda_{ \pm}=1 \pm a \varepsilon+O\left(\varepsilon^{2}\right)$, а соответствуюшие локальные сепаратрисы близки к невозмущенной. Эту близость можно описать следующим 
образом. Уравнение (1.2) при подстановке туда отображения $F_{\varepsilon}$ вьглядит следующим образом:

$$
\psi_{\varepsilon}^{u, s}(t+h)=F_{\varepsilon}\left(\psi_{\varepsilon}^{u, s}(t)\right)=\psi_{\varepsilon}^{u, s}(t)+\varepsilon f\left(\psi_{\varepsilon}^{u, s}(t)\right)+\varepsilon^{2} g\left(\psi_{\varepsilon}^{u, s}(t), \varepsilon\right) .
$$

Так как $h=\ln \lambda_{+}=a \varepsilon+O\left(\varepsilon^{2}\right)$, это уравнение можно переписать в виде

$$
\frac{\psi_{\varepsilon}^{u, s}(t+h)-\psi_{\varepsilon}^{u, s}(t)}{h}=\frac{1}{a} f\left(\psi_{\varepsilon}^{u, s}(t)\right)+\varepsilon \widetilde{g}\left(\psi_{\varepsilon}^{u, s}(t), \varepsilon\right) .
$$

$\Phi$ иксируем произвольное $t_{0} \in \mathbb{R}$. Существуют решения уравнения (2.4) такие, что

$$
\begin{array}{ll}
\psi_{\varepsilon}^{u}(t)=\sigma_{0}(t / a)+O(\varepsilon), & \forall t<t_{0}, \\
\psi_{\varepsilon}^{s}(t)=\sigma_{0}(t / a)+O(\varepsilon), & \forall t>-t_{0} .
\end{array}
$$

Отметим, что можно получить и более точные аппроксимации для сепаратрис, описываемых уравнениями вида (2.4), если искать решения в виде ряда по степеням малого параметра $\varepsilon$ (или $h)$.

2.2. Существование двоякоасимптотических траекторий. Приведем рассуждения, позволяющие показать, что из существования двоякоасимптотической траектории дифференциального уравнения (2.2) вытекает сушествование гомоклинических траекторий отображения (2.1). Доказательства, приведенные ниже, восходят к А. Пуанкаре.

А. Пусть отображсние (2.1) сохраняет площадь. Предположим, что дифференииальное уравнение (2.2) имеет седловую точку $p_{0}$, которой соответствует двоякоасимптотическая траектория $p=\sigma_{0}(t)$. Тогда при всех достаточно мальх в отображение (2.1) имеет по крайней мере две различные гомоклинические траектории, близкие к невозмущенной сепаратрисе.

ДокАЗАТЕЛЬСТво. Фиксируем произвольное $t$ и рассмотрим две точки $A \in W^{u}\left(p_{\varepsilon}\right)$ и $B \in W^{s}\left(p_{\varepsilon}\right)$, лежащие на нормали к невозмущенной сепаратрисе в точке $\sigma_{0}(t)$. Рассмотрим образ криволинейного треугольника $p_{\varepsilon}, A, B$, сторонами которого являются отрезки устойчивой и неустойчивой сепаратрис, а также прямолинейньй отрезок, соединяющий $A$ и $B$. Можно считать, что $A \neq B$, так как в случае $A=B$ сразу имеем одну гомоклиническую точку, а существование второй гомоклинической точки следует из последующих рассуждений. Площади криволинейного треугольника и его образа равны. Поэтому алгебраическая плошадь фигуры П, ограниченной отрезком $W^{u}\left(p_{\varepsilon}\right)$ с конщами в $A$ и $F(A)$, кривой $F([A, B])$, отрезком $W^{s}\left(p_{\varepsilon}\right)$ с конщами в $F(B)$ и $B$, а также прямолинейньм отрезком $[B, A]$, равна нулю.

Убедимся, что $[A, B] \cap F_{\varepsilon}([A, B])=\varnothing$. Действительно, обозначим $C=\sigma_{0}(t)$, тогда для любой точки $p \in[A, B]$

$$
F_{\varepsilon}(p)=p+\varepsilon f(p)+O\left(\varepsilon^{2}\right)=p+\varepsilon f(C)+O\left(\varepsilon^{2}\right),
$$

где мы использовали тот факт, что точки $A$ и $B$ удалены от $C$ на расстояние порядка $\varepsilon$. Таким образом, диффеоморфизм действует на точки отрезка как параллельншй 
перенос на вектор $\varepsilon f(C)$. По построению этот вектор ортогонален отрезку $[A, B]$, а следовательно, пересечение отрезка с его образом невозможно.

Сепаратрисы не имеют самопересечений. Кроме того, рассмотренные отрезки сепаратрис однозначно проектируются (вдоль нормали) на невозмущенную сепаратрису и, следовательно, не имеют других обших точек с $[A, B] \cup F([A, B])$, за исключением конечных точек этих отрезков. Таким образом, равенство нулю площади П означает, что либо имеется четное (с учетом кратности) число точек пересечения устойчивой и неустойчивой сепаратрис (при этом случай одной точки кратности два очевидньм образом невозможен), либо сепаратрисы совпадают.

Отметим, что эти рассуждения не верны, если $\sigma_{0}(t)$ является гетероклинической траекторией, так как в этом случае линии, определявшие в доказательстве фигуру П, не образуют замкнутой ломаной. Несложно построить пример, в котором предельный поток имеет гетероклиническую траекторию, в то время как у отображения гетероклинических точек нет ни при каком положительном $\varepsilon$.

Пусть $F_{\varepsilon}$ является отображением за время $\varepsilon$ системы с гамильтонианом

$$
H(x, y, \varepsilon)=\frac{y^{2}}{2}+\cos x+r \cos 2 x+\varepsilon \sin 2 x .
$$

Предельньй поток описывается гамильтонианом $H(x, y, 0)$ и имеет две седловые точки, соединенные гетероклинической траекторий. Отображение $F_{\varepsilon}$ не имеет при $\varepsilon>0$ гетероклинической траектории, так как его гиперболические неподвижные точки совпадают с седловьми точками гамильтониана $H$, последние же лежат на различных линиях уровня гамильтониана $H$ (см. рис. 4$)$.
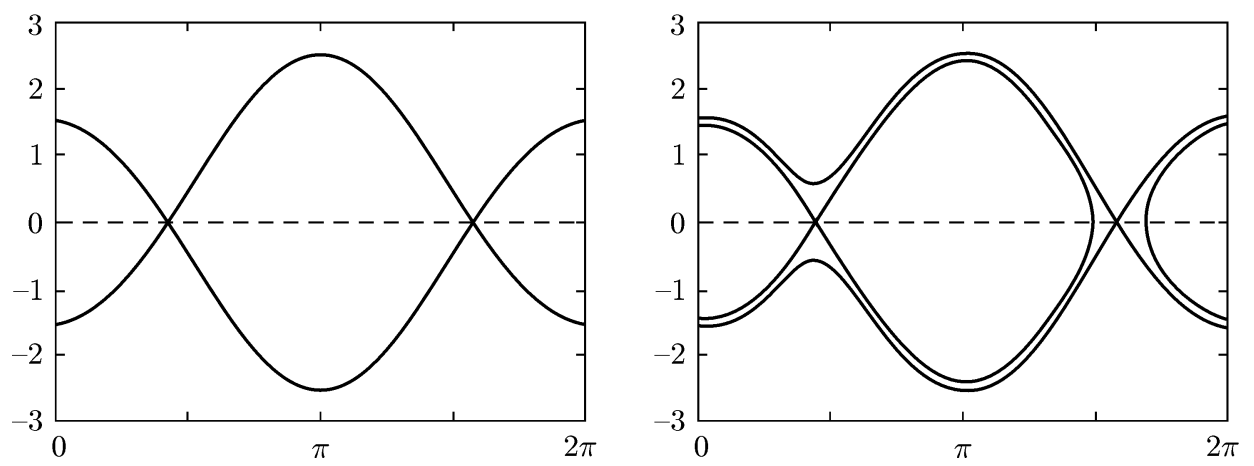

Рис. 4. Исчезновение гетероклинической траектории

В. Другой важньй случай - случай обратимых систем. Напомним, что система называется обратимой, если существует инволюция $R$, назьваемая реверсором, сопрягаюшая отображение $F_{\varepsilon}$ с обратньм:

$$
R \circ F_{\varepsilon}=F_{\varepsilon}^{-1} \circ R, \quad R \circ R=\mathrm{id} .
$$

Множество неподвижных точек отображения инвариантно относительно реверсора. Реверсор переводит неустойчивую сепаратрису в устойчивую и наоборот. Пусть $\ell$ является линией, состояшей из неподвижных точек реверсора. Принято называть $\ell$ линией симметрии. Очевидно, точка пересечения сепаратрисы с $\ell$ является либо гомоклинической, либо гетероклинической точкой. 
Если диффеоморфизм $F_{\varepsilon}$ обратим, а невозмущенная сепаратриса трансверсально пересекает линию симметрии $\ell$, то при всех достаточно малых $\varepsilon$ имеется симметричная двоякоасимптотическая траектория диффеоморфизма, близкая к невозмущенной сепаратрисе.

Гамильтоновы системы часто имеют бесконечно много гомоклинических и гетероклинических траекторий. В процессе исследования обычно приходится выделять одну траекторию из этого множества. Будем назьвать гомоклиническую (гетероклиническую) траекторию, найденную с помощью рассуждений данного пункта, главной. Отметим, что главная траектория всегда принадлежит семейству гомоклинических траекторий $\gamma_{\varepsilon}$ отображения $F_{\varepsilon}$ равномерно близких к невозмушенной гомоклинической траектории $\sigma_{0}$.

2.3. Величины, характеризующие расщепление сепаратрис. Приведем описание величин, с помощью которых принято количественно оценивать расщепление сепаратрис.

А. Угол расщепления сепаратрис $(\alpha)$. Если на фазовом пространстве $S$ задана риманова метрика, то угол расшепления можно определить как угол между векторами, касательными к устойчивой и неустойчивой сепаратрисам в гомоклинической (гетероклинической) точке $p_{h}$.

Недостатком этой величины является неинвариантность: значение угла зависит как от выбора метрики, так и от выбора гомоклинической точки. Отметим, что в теории гамильтоновых систем нет естественным образом выделенной римановой метрики.

В. Амплитуда расщепления $(d)$ равна $\max _{t \in\left[t_{0}, t_{0}+h\right]} \operatorname{dist}\left(\psi_{\varepsilon}^{s}(t), \psi_{\varepsilon}^{u}(t)\right)$, при этом предполагается, что $t_{0}$ отвечает гомоклинической точке: $\psi_{\varepsilon}^{s}\left(t_{0}\right)=\psi_{\varepsilon}^{u}\left(t_{0}\right)$. Эта величина также зависит как от выбора гомоклинической точки, так и от выбора метрики.

С. Гомоклинический инвариант ( $\omega)$ был введен в работе [37]. Формулы

$$
\begin{array}{ll}
\vec{e}_{u}(p)=\dot{\psi}^{u}(t), & p=\psi^{u}(t), \\
\vec{e}_{s}(p)=\dot{\psi}^{s}(t), & p=\psi^{s}(t),
\end{array}
$$

определяют инвариантные касательные векторные поля на $W^{u}$ и $W^{s}$ соответственно. Эти поля не зависят от выбора аддитивной параметризашии $\psi^{u}$ и $\psi^{s}$. Пусть $p_{h} \in$ $W^{u} \cap W^{s}$, гомоклинический инвариант определим как значение формы плошади на паре векторов:

$$
\omega=\Omega\left(\vec{e}_{u}\left(p_{h}\right), \vec{e}_{s}\left(p_{h}\right)\right) .
$$

Если отображение сохраняет форму площади $\Omega$, то гомоклинический инвариант одинаков для всех представителей одной двоякоасимптотической траектории. Кроме того, он инвариантен относительно симплектических замен координат. Это облегчает сопоставление результатов, полученных для одного и того же отображения, записанного в различных координатах.

D. Площадь луночки $(\mathscr{A})$. Рассмотрим отрезки устойчивой и неустойчивой сепаратрис, заключенные между гомоклинической точкой $p_{h}$ и ее образом $F_{\varepsilon}\left(p_{h}\right)$. Если сепаратрисы расщеплены, то эти отрезки имеют только конечное число точек пересечения. Выберем из них две точки пересечения. Отрезки сепаратрис, отсекаемые 
этими точками, образуют луночку. Ее плошадь является для гамильтоновых систем как инвариантом движения, так и инвариантом относительно симплектических замен координат.

Е. Толшина зоны неустойчивости $(\mathscr{W})$. Часто можно бывает показать (например, методами теории КАM), что замькание сепаратрис заключено внутри области, ограниченной инвариантньми кривыми, каждая из которых диффеоморфна окружности. Можно говорить о "последних" инвариантных кривых, ограничивающих так назьваемую зону неустойчивости. В качестве меры расщепления сепаратрис можно взять толшину этой зоны (неинвариантная величина) или ее площадь.

Отметим, что оценка последней величины сложнее, чем предыдуших. По-видимому, размер зоны неустойчивости не является, вообше говоря, даже непрерьвной функщией малого параметра $\varepsilon$. Связь между амплитудой расшепления и толшиной зоны неустойчивости была установлена В. $\Phi$. Лазуткиньм для случая стандартного отображения [55], обобщение этого результата было получено Д. В. Трешевьм [86].

2.4. Локальное выпрямление отображения. Классическим результатом теории дифференциальных уравнений является теорема о том, что векторное поле может быть выпрямлено в окрестности неособой точки. Этот результат можно сформулировать следуюшим образом. Пусть $p_{0}$ является неособой $\left(f\left(p_{0}\right) \neq 0\right)$ точкой дифференциального уравнения $\dot{p}=f(p)$ с аналитической правой частью. Существует такая аналитическая замена координат $p \mapsto y$, определенная в окрестности точки $p_{0}$, что в новых координатах уравнение имеет вид $\dot{y}=e_{1}$, где $e_{1}$ является единичным вектором, направленным вдоль первой координатной оси.

Можно доказать, что аналогичное утверждение справедливо и для отображений, близких к тождественному. Пусть

$$
F_{\varepsilon}(p)=p+\varepsilon f(p)+\varepsilon^{2} g(p, \varepsilon)
$$

и $f\left(p_{0}\right) \neq 0$. Сушествует такая аналитическая замена координат $p \mapsto y$, определенная в не зависящей от $\varepsilon$ окрестности точки $p_{0}$, что в новых координатах отображение имеет вид параллельного переноса вдоль первой координатной оси: $y \mapsto y+\varepsilon e_{1}$. Замена координат может быть выбрана близкой к замене, вьпрямляющей векторное поле $f$.

Это утверждение не зависит от размерности фазового пространства, можно считать, что $p \in \mathbb{R}^{n}\left(\mathbb{C}^{n}\right)$.

Область определения выпрямляющих координат неинвариантна: каждая точка покидает ее после $O\left(\varepsilon^{-1}\right)$ итераций.

Любой объект, инвариантньй относительно отображения $y \mapsto y+\varepsilon e_{1}$, представляет из себя периодическую структуру в направлении первой координатной оси. Этот факт, как станет понятно из последуюшего, дает нам эффективньй инструмент для исследования экспоненциально малого расщепления сепаратрис.

Отметим, что сужение отображения на сепаратрису сопряжено со сдвигом при помощи решения уравнения (2.4). Оказьвается, что для двумерных отображений можно увеличить размерность области определения на единицу и показать, что в полной окрестности сегмента сепаратрисы отображение сопряжено со сдвигом $(T, E) \mapsto$ $(T+h, E)$. Кроме того, можно получить равномерные по $\varepsilon$ оценки на соответствующую замену координат. Это вытекает из следующего утверждения. 
Пусть $p(t ; h)$ является решением конечно-разностного уравнения

$$
p(t+h ; h)=F_{\varepsilon}(p(t ; h)),
$$

аналитическим и равномерно ограниченным в прямоугольнике $D \subset \mathbb{C}$. Предположим, что производная $\dot{p}(t ; h)$ ограничена, ее первая компонента равномерно отделена от нуля в $D$ и сушествует такая постоянная $m_{1}>0$, что

$$
\left\|p\left(t_{1} ; h\right)-p\left(t_{2} ; h\right)\right\| \geqslant m_{1}\left|t_{1}-t_{2}\right|, \quad \forall t_{1}, t_{2} \in D
$$

Лемма 2.4.1 [28]. Существуют постоянные $h_{0}, E_{0}>0$ такие, что при $h \in$ $\left(0, h_{0}\right)$ существует семейство решений уравнения

$$
p(t+h, E ; h)=F_{\varepsilon}(p(t, E ; h)),
$$

обладающих следующими свойствами: $p(t, 0 ; h)=p(t ; h)$, замена переменньх $(t, E) \mapsto p(t, E ; h)$ является аналитическим диффеоморфизмом $D \times\{E \in \mathbb{C}:$ $\left.|E|<E_{0}\right\}$ на образ этого множсества. Производные этого диффеоморфизма ограничены равномерно по $h$. Если отображение $F_{\varepsilon}$ сохраняет площадь, то параметр Е мохет быть выбран так, что отображсение $(t, E) \mapsto p(t, E ; h)$ тожсе сохраняет площадь.

В качестве $p(t ; h)$ мы будем использовать параметризащию сепаратрисы $\psi_{\varepsilon}^{u}(t)$. Требуемые свойства вытекают из ее близости к невозмушенной сепаратрисе.

2.5. Экспоненциальная малость расщепления. Предположим, что дифференциальное уравнение $(2.2)$ имеет две седловые точки $p_{1}$ и $p_{2}$, соединенные двоякоасимптотической траекторией $\sigma_{0}(t)$. Случай, когда $p_{1}=p_{2}$, не исключается. Пусть $\sigma_{0}(t)$ аналитична в полосе $\Pi_{\rho_{0}}=\left\{t \in \mathbb{C}:|\operatorname{Im}(t)|<\rho_{0}\right\}$. Пусть двумерное отображение $F_{\varepsilon}$ вида (2.1) непрерывно зависит от $\varepsilon$ и аналитично в окрестности $\sigma_{0}\left(\Pi_{\rho_{0}}\right)$, не зависящей от $\varepsilon$.

Следуюшая теорема устанавливает квалифищированную оценку на расщепление сепаратрис. Первоначально она была доказана в работе [23], в последующей работе [22] были сняты некоторые несущественные ограничения. Приведенная ниже формулировка является еще более общей: показано, что условие сохранения площади можно опустить, потребовав взамен сушествования главного семейства двоякоасимптотических траекторий.

Теорема 2.5.1 (Э. Фонтик, К. Симо). Пусть точка р принадлежит двоякоасимптотической траектории дифференциального уравнения (2.1). Если существует семейство главных двоякоасимптотических траекторий отображения $F_{\varepsilon}$, близкое $к$ невозмущенной двоякоасимптотической траектории, то при всех $\varepsilon \in\left(0, \varepsilon_{0}\right)$ амплитуда расщепления сепаратрис в окрестности точки р не превосходит

$$
C\left(\rho, \varepsilon_{0}, t_{0}\right) \exp \left(-\frac{2 \pi \rho}{\varepsilon}\right)
$$

при любом $\rho \in\left(0, \rho_{0}\right)$. 
ДокАЗАТЕльство. Пусть сепаратрисы отображения $F_{\varepsilon}$ представлены в параметрическом виде с помошью решений конечно-разностного уравнения (2.4). Пусть $t_{0}$ отвечает главной двоякоасимптотической траектории: $\psi_{\varepsilon}^{u}\left(t_{0}\right)=\psi_{\varepsilon}^{s}\left(t_{0}\right)$. Конечно, $t_{0}$ зависит от $\varepsilon$.

Пусть $D \subset \Pi_{\rho_{0}}$ обозначает прямоугольник, содержаший в себе

$$
\widetilde{D}=\left\{t \in \mathbb{C}:|\operatorname{Im} t|<\rho, t_{0}<\operatorname{Re} t \leqslant t_{0}+h\right\} \subset D .
$$

Можно проверить, что при $t \in D$ вьполнены оценки (2.5) и (2.6), т.е. соответствующие сегменты сепаратрис близки к невозмущенной. Из этой близости вытекает возможность применения леммы $2.4 .1 \mathrm{c} p(t ; h)=\psi_{\varepsilon}^{u}(t)$. Лемма 2.4.1 устанавливает, в частности, существование системы координат $(T, E)$, в которой отображение принимает вид сдвига $(T, E) \mapsto(T+h, E)$. В силу близости к невозмушенной сепаратрисе $\psi_{\varepsilon}^{s}(D)$ принадлежит области определения этих координат при всех $\varepsilon \in\left(0, \varepsilon_{0}\right)$.

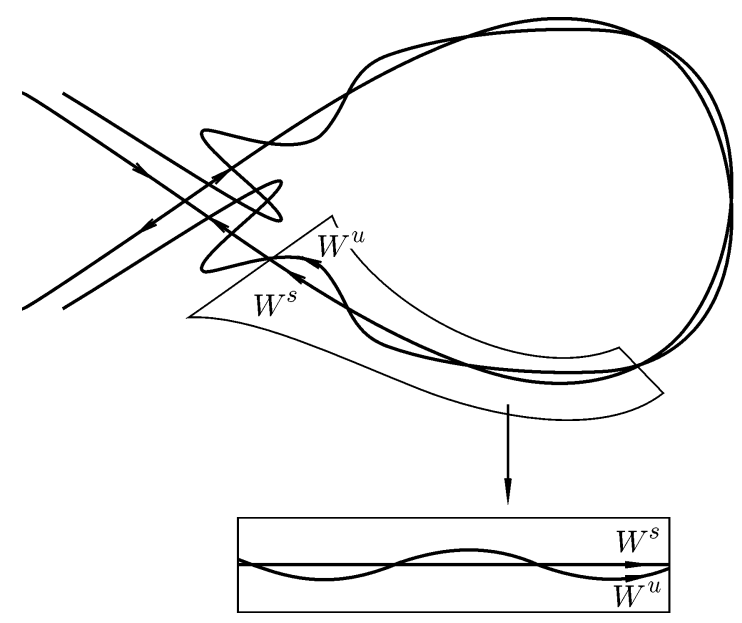

Рис. 5. В координатах $(T, E)$ устойчивая сепаратриса является графиком периодической функции

Расстояние между сепаратрисами можно описать функцией

$$
\Theta(t)=E\left(\psi_{\varepsilon}^{s}(t)\right)-E\left(\psi_{\varepsilon}^{u}(t)\right)=E\left(\psi_{\varepsilon}^{s}(t)\right) .
$$

Несложно проверить, что эта функция периодична:

$$
\Theta(t+h)=E\left(\psi_{\varepsilon}^{s}(t+h)\right)=E\left(F_{\varepsilon}\left(\psi_{\varepsilon}^{s}(t)\right)\right)=E\left(\psi_{\varepsilon}^{s}(t)\right)=\Theta(t) .
$$

Эту функщию можно продолжить по периодичности на полосу П $\rho$. Из леммы 2.4.1 следует, что там эта функция аналитична и ограничена равномерно по параметру. Из равномерной ограниченности этой функции в комплексной полосе вытекает ее экспоненциальная малость на вещественной оси. Чтобы показать это, используем следующую простую лемму, в справедливости которой несложно убедиться разложением в ряд Фурье. 
Лемма 2.5.2 (Лазуткин [52]). Пусть $b>h>0$. Если $h$-периодическая функиия $\theta_{h}$ аналитична и ограничена в полосе $\{t \in \mathbb{C}:|\operatorname{Im} t|<b\}$, а ее среднее значение за период равно нулю, то для всех $t \in \mathbb{R}$

$$
\left|\theta_{h}(t)\right|+h\left|\dot{\theta}_{h}(t)\right| \leqslant 8 e^{-2 \pi b / h} \sup _{|\operatorname{Im} t|<b}\left|\theta_{h}(t)\right| .
$$

Среднее значение $\langle\Theta\rangle$, вообще говоря, отлично от нуля. Применяя лемму 2.5.2, получаем, что для всех вещественных $t$

$$
|\Theta(t)-\langle\Theta\rangle|<\widetilde{C}\left(\rho, \varepsilon_{0}\right) \exp \left(-\frac{2 \pi \rho}{\varepsilon}\right) .
$$

Отметим, что

$$
\Theta\left(t_{0}\right)=E\left(\psi_{\varepsilon}^{s}\left(t_{0}\right)\right)=E\left(\psi_{\varepsilon}^{2}\left(t_{0}\right)\right)=0
$$

Подставляя $t_{0}$ в приведенную вьше оценку, получаем оценку на среднее значение $\langle\Theta\rangle$. Таким образом,

$$
|\Theta(t)|<2 \widetilde{C}\left(\rho, \varepsilon_{0}\right) \exp \left(-\frac{2 \pi \rho}{\varepsilon}\right) .
$$

Так как производные $p(t, E, h)$ равномерно ограничены, аналогичная оценка верна и в исходных координатах.

\section{3. Асимптотика для расщепления сепаратрис отображений}

Оценки сверху для расшепления сепаратрис, приведенные в предыдушем пункте, справедливы при достаточно общих предположениях о свойствах отображения. Получение оценок снизу является более сложной и тонкой задачей. В настоящее время асимптотические формулы для экспоненциально малого расщепления сепаратрис известны для ряда конкретных семейств специального вида. Приведем соответствуюшие результаты.

3.1. Стандартное отображение. Стандартное отображение Чирикова является диффеоморфизмом, определенньм на двумерном торе $\mathbb{T}^{2}=\mathbb{R}^{2} /(2 \pi \mathbb{Z})^{2}$ следуюшими формулами:

$$
S M:(x, y) \mapsto(x+y+\varepsilon \sin x, y+\varepsilon \sin x) .
$$

Стандартное отображение сохраняет плошадь, а также является обратимьм. Одним из реверсоров является инволюция

$$
R:(x, y) \mapsto(2 \pi-x, y+\varepsilon \sin x)
$$

линия $x=\pi$ является соответствующей линией симметрии.

При $\varepsilon=0$ стандартное отображение интегрируемо, а фазовое пространство расслоено на инвариантные окружности $y=$ const. Все точки окружности $y=0$ являются неподвижными. Эта окружность соответствует резонансу. При $\varepsilon>0$ сохраняются только две неподвижные точки. При не слишком больших $\varepsilon$ одна из них является 


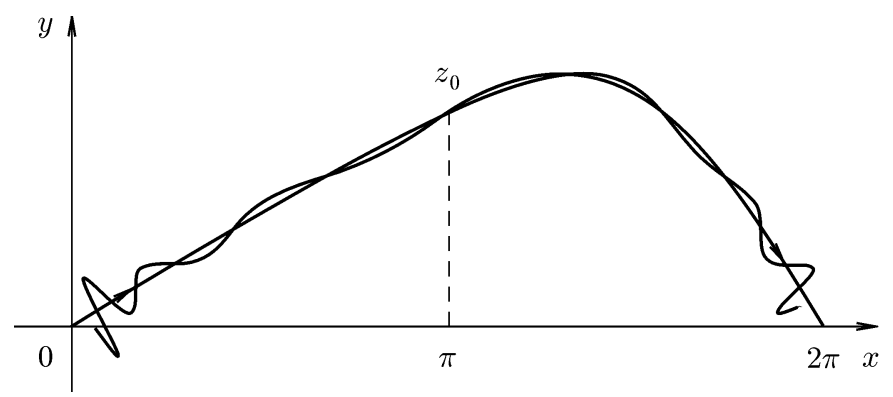

Рис. 6. Расщепление сепаратрис стандартного отображения

гиперболической, а другая - эллиптической. Мультипликаторы гиперболической неподвижной точки $p_{0}=(0,0)$ равны $\lambda$ и $\lambda^{-1}$, где

$$
\lambda=1+\varepsilon / 2+\sqrt{\varepsilon+\varepsilon^{2} / 4} .
$$

Удобно в качестве малого параметра использовать $h=\ln \lambda$. Отметим, что $\varepsilon=4 \operatorname{sh}^{2} \frac{h}{2}$. При малых $h$ сепаратрисы стандартного отображения напоминают сепаратрисы математического маятника (см. рис. 6). Первое пересечение сепаратрисы стандартного отображения с линией симметрии $x=2 \pi$ является главной гомоклинической точкой.

Теорема 3.1.1. Гомоклинический инвариант главной гомоклинической траектории стандартного отображения Чирикова допускает следующее асимптотическое разложение:

$$
\omega \stackrel{\text { as }}{=} \frac{4 \pi}{h^{2}} e^{-\pi^{2} / h}\left(\sum_{n=0}^{\infty} h^{2 n} \omega_{n}\right) .
$$

Символ $\stackrel{\text { as }}{=}$ использован для того, чтобы подчеркнуть асимптотический характер ряда. То есть, если оставить в правой части равенства лишь конечное число слагаемых, ошибка будет иметь тот же порядок, что и первый отброшенный член.

Коэффициенты ряда (3.3) являются вещественными числами, получаюшимися при решении некоторых вспомогательных задач, не зависяших от $\varepsilon$. Процедура нахождения первого коэффициента [58], [57]

$$
\omega_{0}=\left|\Theta_{1}\right|=1118.827706 \ldots
$$

описана в следуюшем пункте.

Приведем несколько значений для остальных коэффициентов, взятых из работы [37],

$$
\begin{aligned}
& \omega_{1}=18.59891 \ldots / 1 !, \quad \omega_{2}=-4.34411 \ldots / 2 ! \\
& \omega_{3}=-4.1829 \ldots / 3 !, \quad \omega_{4}=-4.88 \ldots / 4 !
\end{aligned}
$$

Несмотря на то, что первые коэффициенты разложения убывают, более точные (несколько тысяч десятичных разрядов) вычисления, проведенные К. Симо, позволяют 
предположить, что ряд в правой части (3.3) расходится и принадлежит классу Жевре- 1.

Угол расшепления сепаратрис в $p_{h}$, точке первого пересечения сепаратрис с линией симметрии $x=\pi$,

$$
\alpha \stackrel{\text { as }}{=} \frac{\pi}{h^{2}} e^{-\pi^{2} / h}\left(\sum_{n=0}^{\infty} h^{2 n} c_{n}\right),
$$

где коэффициенты $c_{n}$ могут быть выражены через $\omega_{n}$. В частности,

$$
\omega_{0}=c_{0}, \quad \omega_{1}=c_{1}+\frac{c_{0}}{4}, \quad \omega_{2}=c_{2}+\frac{c_{1}}{4}+\frac{25}{72} c_{0}
$$

Площадь луночки

$$
\mathscr{A} \stackrel{\text { as }}{=} 2 \pi^{-1} e^{-\pi^{2} / h}\left(\sum_{n=0}^{\infty} h^{2 n} \omega_{n}\right) .
$$

Приведем теперь оценки для ширины зоны неустойчивости в окрестности сепаратрисы стандартного отображения Чирикова. Пусть $\Gamma_{0}, \Gamma_{-1}$ и $\Gamma_{1}$ обозначают инвариантные кривые, ограничиваюшие зону неустойчивости. При этом предполагается, что $\Gamma_{0}$ обозначает ту кривую, внутри которой лежит эллиптическая неподвижная точка, а $\Gamma_{ \pm 1}$ делают один оборот вокруг цилиндра. Существуют [55] положительные константы $c_{1}$ и $c_{2}, c_{1}<c_{2}$, такие, что

$$
c_{1} h^{-3} e^{-\pi^{2} / h}<\operatorname{dist}\left(\Gamma_{0}, \Gamma_{ \pm 1}\right)<c_{2} h^{-3} e^{-\pi^{2} / h} .
$$

Таким образом, толшина зоны неустойчивости в $h^{-1}$ раз превосходит угол расщепления.

Стандартное отображение ${ }^{1}$ широко используется в физической литературе для описания движения в окрестности резонансов [88]. "Хаотические” движения в окрестности резонанса были замечены в численных экспериментах Н. Н. Филоненко, Р. З. Сагдеева и Г. М. Заславского [21]. Для описания этих явлений Г. М. Заславский и Н. Н. Филоненко [89] ввели (неявно) сепаратрисное отображение и получили правильные по порядку величины значения его амплитуды. Б. В. Чириков [12] провел более аккуратные численные оценки амплитуды сепаратрисного отображения. Результаты этих вычислений эквивалентны определению постоянной $\omega_{0}$ с относительной погрешностью менее $10 \%$.

В работе [52] был предложен метод, последующее развитие которого позволило получить полное доказательство асимптотических формул для расшепления сепаратрис стандартного отображения и ряда других систем. Приведенная в настоящем пункте теорема была сформулирована в работе [37], полное ее доказательство имеется в [32].

Недавно Д. В. Трещев [84] предложил новьй вариант метода усреднений и вывел с его помощью первьй член формулы для расщепления сепаратрис стандартного отображения Чирикова. Однако полное обоснование этого отсутствует на момент написания настоящего обзора.

\footnotetext{
${ }^{1}$ По поводу истории этой модели и ее физических приложений см. статью [13].
} 
3.2. Постоянная $\Theta_{1}$. Характерной особенностью формул, описьваюших экспоненщиально малое расщепление сепаратрис, является наличие в них постоянного предэкспоненциального множителя, для которого не удается получить конечного выражения через известные универсальные постоянные. Этот множитель можно находить при решении вспомогательной задачи, которую мы опишем на примере полустандартного отображения. Ниже приведено несколько альтернативных определений этого множителя.

Коэффициент $\Theta_{1}$, входяший в асимптотическую формулу для расшепления сепаратрис стандартного отображения, возникает при исследовании расщепления комплексных инвариантных кривых полустандартного отображения $S S M:(u, v) \mapsto\left(u_{1}, v_{1}\right)$,

$$
u_{1}=u+v_{1}, \quad v_{1}=v+e^{u} .
$$

Полустандартное отображение является автоморфизмом $\mathbb{C}^{2}$, сохраняет комплексную форму площади, $d u_{1} \wedge d v_{1}=d u \wedge d v$, и является обратимьм относительно инволюции $R:(u, v) \mapsto\left(u,-v-e^{u}\right)$.

Инвариантные кривые, которые мы обозначим $\Gamma_{-}$и $\Gamma_{+}$, удобно определить параметрически с помощью решений уравнения $\psi(\tau+1)=S S M(\psi(\tau))$. В координатах это уравнение имеет вид системы

$$
\begin{aligned}
& u_{ \pm}(\tau+1)=u_{ \pm}(\tau)+v_{ \pm}(\tau+1) \\
& v_{ \pm}(\tau+1)=v_{ \pm}(\tau)+e^{u_{ \pm}(\tau)}
\end{aligned}
$$

Часто бьвает удобно вместо этой системы исследовать эквивалентное ей конечно-разностное уравнение второго порядка

$$
u_{ \pm}(\tau+1)-2 u_{ \pm}(\tau)+u_{ \pm}(\tau-1)=e^{u_{ \pm}(\tau)}
$$

при этом вторая компонента решения может быть однозначно восстановлена из первого уравнения системы. Уравнение (3.7) имеет два целых решения, однозначно определяемых асимптотикой вида

$$
u_{ \pm}(\tau)=-\ln \frac{\tau^{2}}{2}+w_{ \pm}(\tau), \quad w_{ \pm}(\tau)=O\left(\tau^{-2}\right)
$$

при $\tau$, стремящемся к бесконечности в секторах $D_{+}$и $D_{-}$соответственно, где $D_{-}=$ $\{\tau \in \mathbb{C}:|\arg \tau|>\delta\}, \delta \in(0, \pi)$ - произвольное число, а $D_{+}$является отражением $D_{-}$относительно мнимой оси. Предполагается, что ветви $\ln \frac{\tau^{2}}{2}$ зафиксированы в $D_{ \pm}$таким образом, что функция $\ln \frac{\tau^{2}}{2}$ принимает вешественные значения при вешественных $\tau$ (заметим, что ветви $\ln$, зафиксированные этим условием на $D_{+}$и на $D_{-}$, не совпадают на $\left.D_{+} \cap D_{-}\right)$. Тогда функции $u_{ \pm}$оказываются вещественно-аналитическими. Выбор параметризаций согласован с действием реверсора, который переставляет местами инвариантные кривые: $\Gamma_{+}=R\left(\Gamma_{-}\right)$, в частности, $u_{+}(\tau)=u_{-}(-\tau)$. Сравним эти две функции по модулю $2 \pi i$ на подмножестве нижней компоненты пересечения $D_{+} \cap D_{-}$, а именно, фиксируем $A>1$ и рассмотрим множество

$$
D(A)=\{\tau \in \mathbb{C}:-\pi+\delta<\arg \tau<-\delta, \operatorname{Im} \tau<-A\}
$$


Соответствуюшие сегменты $\Gamma_{ \pm}$оказываются экспоненщиально близкими [56]:

$$
\begin{gathered}
u_{+}(\tau)-u_{-}(\tau)-2 \pi i=O\left(\tau^{2} e^{-2 \pi i \tau}\right), \\
v_{+}(\tau)-v_{-}(\tau)=O\left(\tau e^{-2 \pi i \tau}\right), \quad \tau \in D(A) .
\end{gathered}
$$

До конца настоящего пункта будем подразумевать, что $u_{+}-u_{-}$вычисляется по модулю $2 \pi i$. Рассмотрим нормальную по отношению к $\Gamma_{-}$компоненту разности:

$$
\widehat{\Theta}(\tau)=\operatorname{det}\left(\begin{array}{ll}
\frac{d u_{-}}{d \tau} & u_{+}-u_{-} \\
\frac{d v_{-}}{d \tau} & v_{+}-v_{-}
\end{array}\right)(\tau) .
$$

Постоянную $\Theta_{1}$ можно определить как предел

$$
\Theta_{1} \stackrel{\text { def }}{=} \lim _{\operatorname{Im} \rightarrow-\infty} e^{2 \pi i \tau} \widehat{\Theta}(\tau) .
$$

Отметим, что правая часть стремится к пределу экспоненциально:

$$
\widehat{\Theta}(\tau)=\Theta_{1} e^{-2 \pi i \tau}+O\left(e^{-4 \pi\left(1-\delta^{\prime}\right) i \tau}\right)
$$

при $\operatorname{Im} \tau \rightarrow-\infty$ в $D(A)$.

Приведем еще несколько эквивалентных определений постоянной $\Theta_{1}$. Можно показать [56], что в окрестности сегментов $\Gamma_{+}$и $\Gamma_{-}$полустандартное отображение можно вьпрямить с помошью аналитической симплектической замены координат $(u, v) \mapsto$ $(T, \mathscr{E})$, так что в новых координатах полустандартное отображение имеет вид сдвига вдоль первой координаты $(T, \mathscr{E}) \mapsto(T+1, \mathscr{E})$. Замену координат можно нормировать так, чтобы

$$
T\left(u_{-}(\tau), v_{-}(\tau)\right)=\tau, \quad \mathscr{E}\left(u_{-}(\tau), v_{-}(\tau)\right)=0
$$

Тогда расшепление $\Gamma_{+}$и $\Gamma_{-}$можно описать периодической функцией

$$
\Theta(\tau)=\mathscr{E}\left(u_{+}(\tau), v_{+}(\tau)\right)
$$

При $\operatorname{Im} \tau \rightarrow-\infty$ эта функция стремится к нулю, и ее ряд Фурье имеет вид

$$
\Theta(\tau)=\sum_{k=1}^{\infty} \Theta_{k} e^{-2 \pi i k \tau}
$$

Первый коэффициент этого ряда является интересующей нас постоянной [52]. Действительно, раскладывая правую часть (3.13) в ряд Тейлора с центром в точке $\left(u_{-}, v_{-}\right)(\tau)$ и сохраняя только линейные члены ряда, получаем

$$
\Theta(\tau)=\mathscr{E}\left(u_{-}(\tau), v_{-}(\tau)\right)+\left.\frac{\partial \mathscr{E}}{\partial u}\right|_{\Gamma_{-}}\left(u_{+}-u_{-}\right)+\left.\frac{\partial \mathscr{E}}{\partial v}\right|_{\Gamma_{-}}\left(v_{+}-v_{+}\right)+O_{2},
$$


где $\mathrm{O}_{2}$ обозначает остаточньй член ряда Тейлора, квадратичный по $u_{+}-u_{-}$. Из симплектичности замены переменных и нормировочного условия вытекает, что

$$
\left.\frac{\partial \mathscr{E}}{\partial u}\right|_{\Gamma_{-}}=-\frac{d v_{-}}{d \tau}(\tau),\left.\quad \frac{\partial \mathscr{E}}{\partial v}\right|_{\Gamma_{-}}=\frac{d u_{-}}{d \tau}(\tau) .
$$

Подставляя это в ряд Тейлора, получаем, что

$$
\Theta(\tau)=\widehat{\Theta}(\tau)+O_{2}
$$

При этом малость квадратичного остаточного члена вытекает из оценки (3.9). $\mathrm{У}_{\mathrm{M}-}$ ножая равенство на $e^{2 \pi i}$ и переходя к пределу, убеждаемся, что первьй коэффициент ряда (3.14) совпадает с (3.12).

Функции $u_{ \pm}$являются решениями одного и того же нелинейного уравнения $(3.7)$, поэтому их разность $\xi(\tau)=u_{+}(\tau)-u_{-}(\tau)$ удовлетворяет уравнению

$$
\xi(\tau+1)-2 \xi(\tau)+\xi(\tau-1)=e^{u_{-}(\tau)} \xi(\tau)+r(\tau),
$$

где $r(\tau)=e^{u_{-}(\tau)}\left(e^{\xi(\tau)}-1-\xi(\tau)\right)$. Очевидно, что $r(\tau)=O\left(\tau^{2} e^{-4 \pi i \tau}\right)$ при $\tau \in D(A)$. Функцию $\xi(\tau)$ можно рассматривать как решение неоднородного линейного уравнения. Можно показать [37], [32], что неоднородное уравнение имеет частное решение, допускающее оценку $O\left(\tau^{4} e^{-4 \pi i \tau}\right)$ при $\tau \in D(A)$. Общее решение неоднородного уравнения является суммой частного решения и общего решения однородного уравнения

$$
\phi(\tau+1)-2 \phi(\tau)+\phi(\tau-1)=e^{u_{-}(\tau)} \phi(\tau) .
$$

Общее решение однородного уравнения представимо в виде линейной комбинации с периодическими коэффициентами двух фундаментальных решений. Выберем фундаментальную систему решений однородного уравнения. Удобно в качестве первого решения выбрать $\phi_{1}=\frac{d u_{-}}{d \tau}$, а второе решение определить из условия нормировки

$$
\operatorname{det}\left(\begin{array}{cc}
\frac{d u_{-}}{d \tau}(\tau) & \phi_{2}(\tau) \\
\frac{d v_{-}}{d \tau}(\tau) & \phi_{2}(\tau)-\phi_{2}(\tau-1)
\end{array}\right) \equiv 1
$$

При этом оказьвается, что

$$
\phi_{1}(\tau)=-\frac{2}{\tau}+O\left(\tau^{-3}\right), \quad \phi_{2}(\tau)=-\frac{\tau^{2}}{6}+O(1)
$$

Так как $\xi(\tau)$ стремится к нулю при $\operatorname{Im} \tau \rightarrow-\infty$, то периодические коэффициенты при $\phi_{1}$ и $\phi_{2}$ также стремятся к нулю, и их с хорошей точностью можно заменить на первые коэффициенты соответствуюших рядов Фурье. Таким образом, сушествуют такие постоянные $\mu_{1}$ и $\Theta_{1}$, что

$$
u_{+}(\tau)-u_{-}(\tau)=\mu_{1} e^{-2 \pi i \tau} \phi_{1}(\tau)+\Theta_{1} \phi_{2}(\tau) e^{-2 \pi i \tau}+O\left(e^{-4 \pi\left(1-\delta^{\prime}\right) i \tau}\right) .
$$


Чтобы убедиться в эквивалентности этого определения $\Theta_{1}$ предыдущим, надо подставить последнее выражение в правую часть определения (3.11) и использовать условие нормировки.

Формулу для разности $u_{+}(\tau)-u_{-}(\tau)$ можно несколько упростить, используя асимптотику $\phi_{k}$ :

$$
u_{+}(\tau)-u_{-}(\tau)=-\frac{\Theta_{1}}{6} \tau^{2} e^{-2 \pi i \tau}\left(1+O\left(\tau^{-2}\right)\right)
$$

Можно определить $\Theta_{1}$ как коэффициент в этой асимптотической формуле.

Можно показать [48], [56], что обе функщии $w_{+}(\tau)$ и $w_{-}(\tau)$, входящие в уравнение (3.8), имеют одно и то же асимптотическое разложение

$$
w_{ \pm}(\tau) \stackrel{\text { as }}{=} \sum_{k=1}^{\infty} \frac{a_{k}}{\tau^{2 k}}
$$

при $\tau$, стремяшемся к бесконечности в секторах $D_{ \pm}$соответственно. При больших $m$ $[39],[79]$

$$
a_{m} \sim \frac{(-1)^{m}(2 m+1) !}{(2 \pi)^{2 m}} \alpha
$$

где постоянная $\alpha=1.5034 \ldots$ Эта константа пропорциональна $\Theta_{1}[40],[79]$ :

$$
\Theta_{1}=-i 24 \pi^{3} \alpha
$$

Из этой формулы вытекает, в частности, что постоянная $\Theta_{1}$ является чисто мнимьм числом, так как все $a_{m}$, а следовательно, и $\alpha$ вешественны.

Приведем основные этапы вычислений, позволяющих установить связь между $\Theta_{1}$ и $\alpha$. Рассмотрим для этого вспомогательную функцию

$$
\widetilde{w}(s)=\sum_{m=1}^{\infty} \frac{a_{m}}{(2 m-1) !} s^{2 m-1}
$$

являющуюся преобразованием Бореля формального ряда (3.16). Этот ряд сходится в круге радиуса $2 \pi$ и определяет аналитическую функцию, допускающую аналитическое продолжение на комплексную плоскость переменной $s$ с вертикальными разрезами, идущими из точки $2 \pi i$ на $+i \infty$ и из точки $-2 \pi i$ на $-i \infty$. Из асимптотики $a_{m}$ следует, что точки $\pm 2 \pi i$ являются особыми, при этом в их окрестности

$$
\widetilde{w}(s) \sim \frac{4 \pi^{2} \alpha}{(s \mp 2 \pi i)^{3}} .
$$

Можно показать [49], что функции $w_{ \pm}$можно восстановить, применяя преобразование Лапласа:

$$
w_{ \pm}(\tau)=\int_{0}^{ \pm \infty} e^{-\tau s} \widetilde{w}(s) d s
$$


где интеграл берется вдоль положительной и отрищательной вешественньх полуосей соответственно. Взяв разность этих интегралов, получаем

$$
w_{+}(\tau)-w_{-}(\tau)=\int_{-\infty}^{+\infty} e^{-\tau s} \widetilde{w}(s) d s
$$

где интегрирование ведется вдоль вещественной оси. При $\operatorname{Im} \tau<0$ контур можно продеформировать в верхнюю полуплоскость, при этом основной вклад в интеграл дает особенность при $s=2 \pi i$ :

$$
\begin{aligned}
w_{+}(\tau)-w_{-}(\tau) & \approx 2 \pi i \operatorname{Res}_{s=2 \pi i} e^{-\tau s} \widetilde{w}(s) \\
& \approx 2 \pi i \cdot 4 \pi^{2} \alpha \operatorname{Res}_{s=2 \pi i} \frac{e^{-\tau s}}{(s-2 \pi i)^{3}}=4 \pi^{3} i \alpha \tau^{2} e^{-2 \pi i \tau}
\end{aligned}
$$

Таким образом,

$$
u_{+}(\tau)-u_{-}(\tau) \approx 4 \pi^{3} i \alpha \tau^{2} e^{-2 \pi i \tau}
$$

Сравнивая это выражение с (3.15), получаем желаемую формулу (3.17).

Численные методы нахождения $\Theta_{1}$. По всей видимости, постоянная $\Theta_{1}$ не имеет явного конечного выражения через известные постоянные. Поэтому представляет интерес обсудить численные методы, позволяющие вычислять подобные величины с разумной точностью. Каждое из данных выше определений может быть использовано для нахождения численного значения $\Theta_{1}$. Приведем здесь описание только двух алгоритмов.

I. Как было показано выше,

$$
\Theta_{1}=e^{2 \pi i \tau} \widehat{\Theta}(\tau)+O\left(e^{-2 \pi i(1-\delta) \tau}\right) .
$$

Положим $\tau=-i \sigma$ и возьмем первое слагаемое в правой части в качестве приближения для $\Theta_{1}$. Элементы матрицы (3.11) можно вычислить следуюшим образом. Фиксируем $n$ (порядка 50) и вычисляем $u_{-}(\tau-n)$ и его производную $\frac{d u_{-}}{d \tau}(\tau-n)$ по асимптотическим формулам (3.8), (3.16), прекращая суммирование на самом маленьком члене ряда. Восстанавливаем вторую компоненту, а затем применяем $n$ раз $S S M$ и $S S M^{\prime}$. Точку на устойчивой сепаратрисе получаем путем применения реверсора $R$.

Оптимальное значение $\sigma$ выбирается из следуюших соображений. С ростом $\sigma$ экспоненциально убывает погрешность, связанная с пренебрежением вторьм слагаемым, однако одновременно растет погрешность выгисления первого слагаемого, так как неизбежные погрешности округления умножаются на экспоненциально растуший по $\sigma$ коэффициент. Оптимум достигается, когда вклады от этих погрешностей имеют одинаковый порядок. При 16-разрядной арифметике этот способ позволяет найти примерно 7-8 правильных десятичных разрядов $\Theta_{1}$.

II. Подставляя

$$
u(\tau)=-\ln \frac{\tau^{2}}{2}+\sum_{k=1}^{\infty} \frac{a_{k}}{\tau^{2 k}}
$$


в уравнение (3.7) и собирая слагаемые одного порядка по $\tau$, несложно вьписать [39] рекуррентные соотношения, определяюшие коэффищиенты $a_{k}$. Эти коэффищиенты быстро растут, поэтому для проведения вычислений необходимо произвести перенормировку, положив

$$
\alpha_{m}=(-1)^{m} \frac{(2 \pi)^{2 m}}{(2 m+1) !} a_{m} .
$$

Не слишком сложно вьписать рекуррентные соотношения для коэффициентов $\alpha_{m}$, с их помошью определить значительное число численных значений для первых коэффициентов и, таким образом, получить оценку для предела $\alpha_{m} \rightarrow \alpha$. Для нахождения $\Theta_{1}$ используется соотношение (3.17).

Эффективность данного метода может быть значительно улучшена на основании анализа, проведенного в [36].

3.3. Обобщенное стандартное отображение. Естественным обобщением стандартного отображения Чирикова является диффеоморфизм $(x, y) \mapsto\left(x_{1}, y_{1}\right)$, где

$$
x_{1}=x+y_{1}, \quad y_{1}=y+\varepsilon P(x),
$$

$P(x)$ является либо тригонометрическим

$$
P(x)=\sum_{k=1}^{n} a_{k} \sin \left(k x+b_{k}\right),
$$

либо алгебраическим

$$
P(x)=\sum_{k=0}^{n} a_{k} x^{k}
$$

полиномом. Приведем асиптотические формулы, описьваюшие расщепления сепаратрис подобных отображений.

Удобно сделать замену переменных

$$
X=x, \quad Y=y / \mu, \quad \mu=\sqrt{\varepsilon} .
$$

Эта замена не является симплектической. Значение гомоклинического инварианта не сохраняется при этой замене. Так как якобиан замены постоянен, значение гомоклинического инварианта умножается на $\mu^{-1}$. В новых переменных обобшенное стандартное отображение принимает вид $(X, Y) \mapsto\left(X_{1}, Y_{1}\right)$,

$$
X_{1}=X+\mu Y_{1}, \quad Y_{1}=Y+\mu P(X) .
$$

Траектории отображения при малых $\mu$ похожи на траектории системы дифференциальных уравнений

$$
\dot{X}=Y, \quad \dot{Y}=P(X) .
$$

Несложно проверить, что неподвижные точки отображения (3.22) являются положениями равновесия для системы (3.23) и наоборот. Причем гиперболическим неподвижным точкам отображения отвечают седловые точки системы дифференциальных уравнений. Неподвижные точки имеют вид $(x, 0)$, где $x$ - корень уравнения $P(x)=0$. 
При этом неподвижная точка является гиперболической тогда и только тогда, когда $P^{\prime}(x)>0$.

Пусть $p_{k}=\left(x_{k}, 0\right), k=1,2,-$ две (необязательно различные) седловые точки, соединенные двоякоасимптотическим (сепаратрисньм) решением $\left(X_{0}(t), Y_{0}(t)\right)$ системы (3.23):

$$
\lim _{t \rightarrow-\infty} X_{0}(t)=x_{1}, \quad \lim _{t \rightarrow+\infty} X_{0}(t)=x_{2}
$$

Для простоты предположим, что $X_{0}(t)$ является четной или нечетной функцией.

Существует такое число $\rho>0$, что $X_{0}(t)$ аналитична в полосе $|\operatorname{Im} t|<\rho$ и имеет особенности на границе этой полосы. В силу свойств четности особенности расположены симметрично. Пусть эти особенности обозначены $t_{k}=i \rho+\beta_{k}, k=1, \ldots, m$ $\left(0 \leqslant \beta_{1}<\cdots<\beta_{m}\right)$, а все остальные особенности, лежашие на границе полосы, совпадают с $-t_{k}, \bar{t}_{k}$ или $-\bar{t}_{k}$.

Если $X_{0}(t)$ соответствует гомоклинической траектории и $m=1$, то требование четности $X_{0}(t)$ может быть опушено.

При сделанных вьше предположениях сушествует главная двоякоасимптотическая траектория $\gamma$, одна из точек которой

$$
p_{h}=\left(X_{0}(0), Y_{0}(0)\right)+O\left(\mu^{2}\right) .
$$

Справедлива асимптотическая (при $\mu \rightarrow 0$ ) формула

$$
\omega=-\frac{4 \pi|\theta|}{d \mu^{\nu}} \exp \left(-\frac{2 \pi \rho}{\mu}\right) \sum_{k=1}^{m} c_{k} \cos \frac{2 \pi \beta_{k}}{\mu}+O\left(\mu^{-\nu+\delta} \exp \left(-\frac{2 \pi \rho}{\mu}\right)\right)
$$

где

$$
c_{k}= \begin{cases}1, & \beta_{k}=0 \\ 2, & \beta_{k} \neq 0\end{cases}
$$

В случае, когда $P(x)=\sum_{k=1}^{n} a_{k} \cos \left(k x+b_{k}\right)$ является тригонометрическим полиноMOM,

$$
\left.\nu=3, \quad d=n^{2}, \quad \delta \in\right] 0,1 /(2 n+2)\left[, \quad|\theta|=\left|\Theta_{1}\right|=1118.827706 \ldots\right.
$$

Если же $P(x)=\sum_{k=0}^{n} a_{k} x^{k}-$ алгебраический полином, то

$$
\left.\nu=3+4 /(n-1), \quad d=a_{n}^{2 /(n-1)}, \quad \delta \in\right] 0,1 /(2 n)[, \quad \theta \equiv \Theta(n),
$$

а значение $\Theta(n)$ зависит только от степени полинома:

\begin{tabular}{|c|c|c|c|c|c|}
\hline$n$ & 2 & 3 & 4 & 5 & 6 \\
\hline$\Theta(n)$ & $2.47 \ldots \times 10^{6}$ & $7.94 \ldots \times 10^{3}$ & $930.15 \ldots$ & $279.56 \ldots$ & $125.29 \ldots$ \\
\hline
\end{tabular}

Угол расщепления сепаратрис

$$
\alpha\left(p_{h}\right) \sim \frac{\omega}{\dot{X}_{0}(0)^{2}+\dot{Y}_{0}(0)^{2}}
$$


с той же погрешностью, что и в формуле (3.24). Сушествует $\gamma^{\prime}$ - вторая главная траектория, площадь соответствующей луночки

$$
\mathscr{A}=\frac{\mu^{2}|\omega|}{2 \pi^{2}}+O\left(\mu^{-\nu+\delta+2} \exp \left(-\frac{2 \pi \rho}{\mu}\right)\right) .
$$

Приведенные асимптотические формулы не являются равномерными по коэффициентам полинома $P$ даже при фиксированном $n$. Константа в оценке остаточного члена зависит от выбора $\delta$. Приведенное в [38] доказательство не является полным. Для стандартного отображения Чирикова соответствующее полное доказательство имеется в [32].

Представляет интерес выписать отдельно асимптотические формулы для некоторых примечательных случаев. Пусть $P(x)=x-x^{n}$. В этом случае единственная гиперболическая неподвижная точка расположена в начале координат. Сепаратриса предельного потока может быть вьписана явно, ее первая компонента равна

$$
X_{0}(t)=\left(\frac{n+1}{\operatorname{ch}((n-1) t)+1}\right)^{1 /(n-1)} .
$$

Ближайшая к вешественной оси особенность $t_{0}=\frac{\pi}{n-1}$ является точкой ветвления конечного порядка (при $n \geqslant 4$ ) или полюсом (при $n=2,3$ ).

В силу обратимости стандартного отображения одна из гомоклинических точек лежит на линии симметрии $y=0$. Ее первая координата равна $x_{h}=X_{0}(0)+O\left(\mu^{2}\right)=$ $((n+1) / 2)^{1 /(n-1)}+O\left(\mu^{2}\right)$. Формула (3.24) принимает вид

$$
\omega \sim \frac{4 \pi|\Theta(n)|}{\mu^{3+4 /(n-1)}} \exp \left(-\frac{2 \pi^{2}}{(n-1) \mu}\right) .
$$

Постоянные $\Theta(n)$ возникают при исследовании расщепления сепаратрис параболической неподвижной точки отображений $(u, v) \mapsto\left(u_{1}, v_{1}\right)$,

$$
u_{1}=u+v_{1}, \quad v_{1}=v-u^{n} .
$$

При этом все определения полностью аналогичны случаю полустандартного отображения и постоянной $\Theta_{1}$.

Частным случаем является сохраняющее площадь отображение Эно (Henon), которое можно записать в виде

$$
(x, y) \mapsto(x+y+\varepsilon x(1-x), y+\varepsilon x(1-x)) .
$$

Отметим, что любой сохраняюший площадь квадратичньй диффеоморфизм плоскости с двумя неподвижными точками может быть приведен к этому виду линейньм преобразованием. Отображение Эно является обратимьм, среди реверсоров выделяется

$$
R:(x, y) \mapsto(x-y,-y),
$$


имеюший линию симметрии $y=0$. При $\varepsilon>0$ начало координат является гиперболической неподвижной точкой. Гомоклинический инвариант главной гомоклинической траектории равен

$$
\omega \stackrel{\mathrm{as}}{=} \frac{\pi}{h^{6}} \exp \left(-\frac{\pi^{2}}{h}\right) \sum_{k=0}^{\infty} \omega_{k} h^{2 k}, \quad \omega_{0}=\Theta(2)=2.47 \ldots \cdot 10^{6} .
$$

Первый член этого разложения был получен в работе [35] (см. также [38]), полное асимптотическое разложение - в [11], [10].

Случай отображения (3.19) при $P(x)=\sin x+2 r \sin 2 x$ был рассмотрен в работе [53]. Для угла расщепления сепаратрис были получены следуюшие формулы:

$$
\begin{aligned}
& \alpha=-\frac{\pi\left|\Theta_{1}\right|}{2 \varepsilon} \exp \left(-\frac{2 \pi \rho}{\sqrt{\varepsilon}}\right) \cos \frac{2 \pi \beta(r)}{\sqrt{\varepsilon}}+O\left(e^{-\frac{2 \pi \rho}{\sqrt{\varepsilon}} \varepsilon^{-1+1 / 24-\delta}}\right), \quad r>0, \\
& \alpha=-\frac{\pi\left|\Theta_{1}\right|}{4 \varepsilon} \exp \left(-\frac{2 \pi \rho}{\sqrt{\varepsilon}}\right)\left(1+O\left(\varepsilon^{1 / 24-\delta}\right)\right), \quad-\frac{1}{4} \leqslant r \leqslant 0, \\
& \alpha=-\frac{\pi\left|\Theta_{1}\right|}{(2-4 r-1 / 4 r) \varepsilon} \exp \left(-\frac{2 \pi \rho}{\sqrt{\varepsilon}}\right)\left(1+O\left(\varepsilon^{1 / 24-\delta}\right)\right), \quad r<-\frac{1}{4},
\end{aligned}
$$

где

$$
\begin{aligned}
& \rho(r)= \begin{cases}\frac{\pi}{2 \sqrt{4 r+1}}, & r>0, \\
\frac{\arccos (-8 r-1)}{2 \sqrt{4 r+1}}, & -\frac{1}{4}<r<0, \\
1, & r=-\frac{1}{4}, \\
\frac{\arccos (-1 / 4 r)}{2 \sqrt{-r+1 / 16 r}}, & r<-\frac{1}{4},\end{cases} \\
& \beta(r)=\frac{\ln (\sqrt{4 r+1}+\sqrt{4 r})}{\sqrt{4 r+1}}, \quad r>0 .
\end{aligned}
$$

Интересной особенностью этих формул является наличие в асимптотике осциллирующего множителя при $r>0$. Это означает, что угол пересечения меняет знак и при некоторых значениях $\varepsilon$ обрашается в нуль. Однако из этого не следует, что при этих значениях малого параметра сепаратрисы нерасщеплены, следует только то, что в окрестности этих значений происходят бифуркации гомоклинических точек.

Отметим особо, что при $r=-1 / 4$ неподвижная точка является параболической. Несмотря на это, она обладает устойчивым и неустойчивьм инвариантньми многообразиями, расщепление которых описьвается такой же формулой, как и в случае гиперболической неподвижной точки. Асимптотические формулы при этом зависят от $r$ непрерьвно, но функция $\rho(r)$ имеет скачок производной при $r=-1 / 4$.

Обобщение этих результатов на случай произвольного тригонометрического полинома второго порядка было получено в работе [34]. 
3.4. "Стохастическая паутина". Еще одно отображение, представляющее интерес с точки зрения его возможных приложений к описанию физических явлений, это отображение “с подкручиванием" $M_{\varphi}(\mu):(x, y) \mapsto\left(x_{1}, y_{1}\right)$, где

$$
\begin{aligned}
& x_{1}=(x+\mu \sin y) \cos \varphi+y \sin \varphi, \\
& y_{1}=-(x+\mu \sin y) \sin \varphi+y \cos \varphi .
\end{aligned}
$$

Это отображение было введено в работе [90] как модель движения электрона в плазме. K этому отображению сводятся уравнения движения электрона в вертикально направленном магнитном поле под воздействием горизонтально распространяющихся электростатических импульсов. Значения угла $\varphi$, соизмеримые с $2 \pi$, отвечают резонансам между гироскопической частотой врашения заряженной частицы и частотой импульсов. В частности, при $\varphi=\pi / 2$ имеет место резонанс $1: 4$. Сепаратрисы гиперболических периодических траекторий образуют бесконечную сеть, покрьвающую плоскость $(x, y)$ (см. рис. 7$)$.

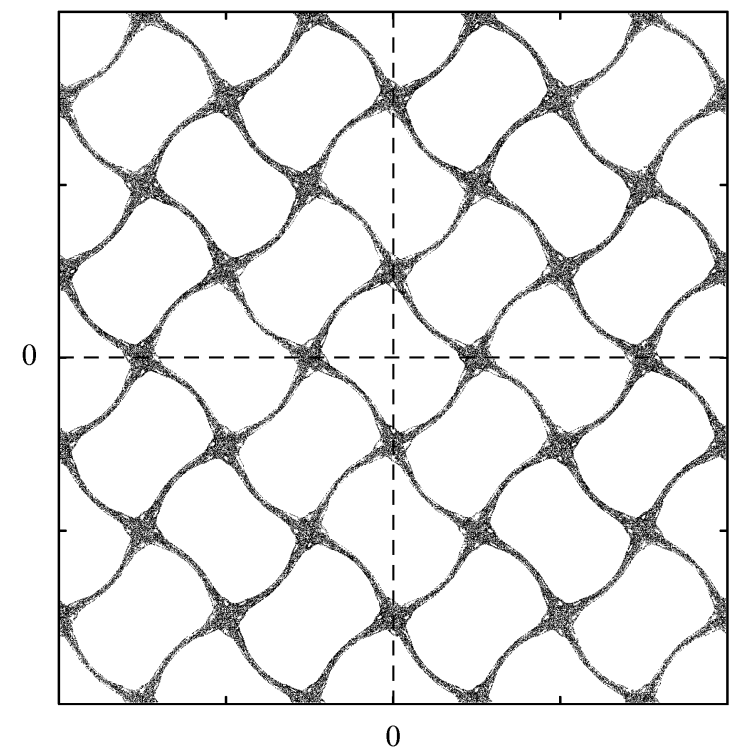

Рис. 7. "Стохастическая паутина", $\mu=1.3, \varphi=\pi / 2$

Опишем эти сепаратрисы подробнее. Отображение $M_{\pi / 2}(\mu):(x, y) \mapsto\left(x_{1}, y_{1}\right)$,

$$
\begin{aligned}
& x_{1}=y, \\
& y_{1}=-(x+\mu \sin y),
\end{aligned}
$$

имеет гиперболическую периодическую траекторию $(\pi, 0) \mapsto(0,-\pi) \mapsto(-\pi, 0) \mapsto$ $(0, \pi) \mapsto(\pi, 0)$. Для исследования расшепления сепаратрис точки периода 4 обычно рассматривают четвертую итерацию отображения, которая при малых $\mu$ близка 
к тождественному отображению. Однако отображение $M_{\pi / 2}(\mu)$ обладает дополнительной симметрией: оказывается, что из $M_{\pi / 2}^{4}(\mu)$ можно извлечь квадратный корень, тоже являющийся отображением, близким к тождественному. Действительно, рассмотрим вспомогательное отображение $\widetilde{M}(\mu):(x, y) \mapsto\left(x_{1}, y_{1}\right)$,

$$
\begin{aligned}
& x_{1}=x+\mu \sin y, \\
& y_{1}=y-\mu \sin x_{1} .
\end{aligned}
$$

Непосредственным вычислением можно убедиться, что

$$
M_{\pi / 2}^{4}(\mu)=\widetilde{M}^{2}(\mu)=\widetilde{M}(2 \mu)+O\left(\mu^{2}\right) .
$$

Таким образом, расщепление сепаратрис у отображения $M_{\pi / 2}(\mu)$ в точности такое же, как у отображения $\widetilde{M}(\mu)$, в то время как поверхностный анализ подсказьвает, что расщепление могло бы быть таким же, как у $\widetilde{M}(2 \mu)$. Подобная ошибка встречается в физической литературе. Как показьвает нижеследуюшая формула при малых $\mu$, расшепление сепаратрис $M_{\pi / 2}(\mu)$ квадратично мало по сравнению с расщеплением $\widetilde{M}(2 \mu)$. Для гомоклинического инварианта главной гомоклинической траектории отображения $\widetilde{M}(\mu)$ имеет место асимптотическая формула [38]:

$$
\omega \sim \frac{4 \pi\left|K_{1}\right|}{\mu^{2}} \exp \left(-\frac{\pi^{2}}{\mu}\right)
$$

где $\left|K_{1}\right|=69.188936 \ldots$ Угол пересечения сепаратрис в гомоклинической точке, лежашей на линии симметрии $y=x$, связан с гомоклиническим инвариантом формулой $\alpha \sim \omega / 2$. Разложение для гомоклинического инварианта этого отображения по степеням $h^{2}$ было получено в [66].

Этот пример показьвает, что изменение старших членов разложения отображения по степеням малого параметра может существенно сказьваться на порядке величины расшепления сепаратрис.

3.5. Расщепление сепаратрис около бифуркации. Мы уже отмечали во введении, что экспоненциально малое расщепление сепаратрис встречается в задачах о бифуркациях неподвижных точек отображений. Рассмотрим одну из типичных бифуркаций коразмерности один в пространстве сохраняющих площадь отображений.

Пусть аналитическое однопараметрическое семейство сохраняюших площадь отображений $F$ раскладьвается в ряд Тейлора по параметру $\varepsilon$ :

$$
F(p, \varepsilon)=F_{0}(p)+\varepsilon F_{1}(p)+\varepsilon^{2} F_{2}(p)+\cdots .
$$

Предположим, что при $\varepsilon=0$ происходит рождение пары неподвижных точек. В случае общего положения, который мы опишем несколько ниже, одна из неподвижных точек оказывается гиперболической, а другая - эллиптической. Сепаратрисы гиперболической неподвижной точки образуют маленькую петлю вокруг эллиптической точки. 
Сформулируем предположения о свойствах отображения $F$ в подходяшей системе координат. Предположим, что отображение $F_{0}$ приведено с помошью сохраняющей площадь замены координат к следующему виду:

$$
F_{0}(x, y)=\left(x+y+\cdots, y-b_{2} x^{2}+\cdots\right), \quad b_{2}>0 .
$$

Точки обозначают члены ряда Тейлора, имеющие более высокий порядок. Кроме того, пусть

$$
F_{1}(0,0)=\left(a_{0}, b_{0}\right), \quad b_{0}>0 .
$$

Тогда при всех не слишком больших $\varepsilon>0$ в окрестности начала координат отображение $F$ имеет две неподвижные точки, одна из которых является гиперболической. Мультипликатор гиперболической неподвижной точки равен

$$
h=\ln \lambda=\sqrt[4]{4 b_{0} b_{2}}+O(\sqrt{\varepsilon}) .
$$

Удобно использовать $h$ в качестве нового параметра вместо $\varepsilon$. Качественно сепаратрисы гиперболической неподвижной точки вьглядят как сепаратрисы автономной гамильтоновой системы, описьваемой гамильтонианом $H=y^{2} / 2+x^{3} / 3-\varepsilon x$. В отличие от этой системы, сепаратрисы отображения $F$ могут иметь трансверсальные пересечения. Гомоклинический инвариант главной гомоклинической траектории описьвается асимптотической формулой [33]

$$
\omega=\frac{4 \pi\left|\Theta_{1}\left(F_{0}\right)\right|}{h^{2}} e^{-2 \pi^{2} / h}+O\left(e^{-2 \pi^{2} / h}\right) .
$$

В этой формуле предэкспоненщиальньй множитель $\Theta_{1}\left(F_{0}\right)$ зависит только от отображения $F_{0}$. Если этот множитель отличен от нуля, то расщепления сепаратрис трансверсально при всех достаточно малых $\varepsilon$. Естественно, что если отображение $F_{0}$ интегрируемо (т.е. имеет нетривиальный аналитический первьй интеграл в окрестности начала координат), то $\Theta_{1}\left(F_{0}\right)=0$. Численные эксперименты показывают, что $\Theta_{1}\left(F_{0}\right)$ может обрашаться в нуль и для неинтегрируемых отображений. Однако в “типичном" случае этот множитель отличен от нуля.

Недавно было аналитически доказано, что в случае квадратичного сохраняющего площадь отображения (Henon map) $\Theta_{1}\left(F_{0}\right) \neq 0[36]$.

\section{4. Системы с быстро вращающейся фазой}

Отображения, близкие к тождественному, можно рассматривать как отображения последования за период по времени для неавтономных векторных полей. Такой подход позволяет применить для исследования расщепления сепаратрис отображений методы, разработанные для систем дифференциальных уравнений. Особьй интерес для нас представляет здесь метод усреднений.

Рассмотрим систему дифференщиальных уравнений вида

$$
\dot{x}=\varepsilon f(x, \varphi, \varepsilon), \quad \dot{\varphi}=\omega(x)+\varepsilon g(x, \varphi, \varepsilon),
$$

где $x \in \mathbb{R}^{n}, \varphi \in \mathbb{S}^{1}, \varepsilon \in\left[0, \varepsilon_{0}\right]$. Вектор $x$ назьвают вектором медленных переменных, $\varphi$ - скалярная угловая переменная, называемая фазой. Правые части системы предполагаются $2 \pi$-периодическими по $\varphi$. Предположим, что частота $\omega(x)$ не обращается 
в нуль в рассматриваемой области. В этом случае система имеет два характерных масштаба времени, отношение которых описьвается малым параметром $\varepsilon$.

Для исследования систем подобного вида часто применяют метод усреднений [7]. Метод усреднений состоит в построении последовательности замен координат, последовательно исключающих зависимость правой части уравнений от фазы в старших порядках по $\varepsilon$. Классическим результатом теории усреднений является следующее утверждение. Для любого целого $m$ можно построить близкую к тождественной $2 \pi-$ периодическую по $\varphi$ замену переменных

$$
x=y+\varepsilon U(y, \psi, \varepsilon), \quad \varphi=\psi+\varepsilon V(y, \psi, \varepsilon),
$$

$U=O(1), V=O(1)$, такую, что уравнения для новых переменных содержат фазу лишь в членах порядка $\varepsilon^{m+1}$ :

$$
\dot{y}=\varepsilon(F(y, \varepsilon)+\alpha(y, \psi, \varepsilon)), \quad \dot{\psi}=\Omega(y, \varepsilon)+\varepsilon \beta(y, \psi, \varepsilon),
$$

где $\alpha=O\left(\varepsilon^{m}\right), \beta=O\left(\varepsilon^{m}\right), F=\langle f\rangle+O(\varepsilon), \Omega=\omega+O(\varepsilon)$, а угловые скобки обозначают усреднение по фазе $\varphi$. Функции $U, V-$ многочлены степени $m$ по $\varepsilon$.

Если исходная система является (локально) гамильтоновой, то замены координат можно сделать каноническими. Для этого следует строить замены координат с помошњю производящих функщий.

Отбрасьвая в правых частях (4.3) малые члены $\alpha$ и $\beta$, получают укороченную систему, решения которой аппроксимируют решения (4.3), а значит и исходной системы (4.1), на интервале времени $0 \leqslant t \leqslant 1 / \varepsilon$ с точностью $O\left(\varepsilon^{m}\right)$ для $x$ и $O\left(\varepsilon^{m-1}\right)$ для $\varphi$. Укороченная система проше для исследования, так как в ней медленное движение полностью отделено от быстрого. Если исходная система является неавтономной гамильтоновой системой с одной степенью свободы и периодическим по времени гамильтонианом (подобные системы принято называть системами с полутора степенями свободы), то укороченная система является автономной, а ее сепаратрисы, если таковые имеются, нерасшеплены, так как принадлежат линии уровня укороченного гамильтониана. Так как сепаратрисы гладко зависят от системы, то возмущение порядка $O\left(\varepsilon^{m}\right)$ приводит к расщеплению сепаратрис того же порядка. В силу произвольности $m$ расщепление оказьвается меньше любой степени малого параметра.

4.1. Точность разделения движений. Если устремить $m$ к бесконечности, то для замены переменных (4.2) получатся ряды, которые в обшем случае расходятся. А.И. Нейштадт [65] заметил, что в аналитическом случае, увеличивая число шагов метода усреднений обратно пропорционально значению $\varepsilon$, можно разделить быстрые и медленные движения с экспоненщиально высокой точностью. Сформулируем соответствуюшую теорему.

Пусть правые части (4.1) - вещественно-аналитические функции в комплексной $\delta$-окрестности $D+\delta$ вешественной области $D=G \times \mathbb{S}^{1}\left(x \in G, \varphi \in \mathbb{S}^{1}\right)$, удовлетворяюшие оценкам

$$
|f|<C, \quad|g|<C, \quad c^{-1}<|\omega|<C,
$$

где $\delta, c, C$ - положительный постоянные, $G$ - область в $\mathbb{R}^{n}$. 
Теорема 4.1 .1 (А.И. Нейштадт [65]). При $(y, \psi) \in D+1 / 2 \delta, 0<\varepsilon<\varepsilon_{1}$ существует вещественно-аналитическая $2 \pi$-периодическая по $\psi$ замена переменных вида (4.2), приводящая систему (4.1) к виду (4.3) с әкспоненииально мальми $\alpha u \beta$,

$$
\begin{gathered}
|\alpha|+|\beta|<c_{2} \exp \left(-c_{1} / \varepsilon\right), \\
|U|+|V|<c_{3}, \quad|F-\langle f\rangle|+|\Omega-\omega|<c_{4} \varepsilon .
\end{gathered}
$$

Постоянные $\varepsilon_{1}, c_{1}, c_{2}, c_{3}, c_{4}$ зависят от $\varepsilon_{0}, \delta, c, C$. Если система (4.1) гамильтонова, то замена может быть выбрана канонической.

Искомая замена строится в виде композиции большого числа последовательно определяемых замен, уничтожающих зависимость от фазы в членах все более высокого порядка. Каждая замена может быть выбрана так, что зависящие от фазы члены убывают в геометрической прогрессии (по номеру замены), однако область определения замены на каждом шагу сужается на величину const · $\varepsilon$, так как для производных используются оценки Коши. В силу конечности области можно сделать не более $O\left(\varepsilon^{-1}\right)$ шагов, что приводит к оценке $\alpha, \beta=O\left(e^{-c / \varepsilon}\right)$.

В частности, теорема применима к системам, в которых роль фазы играет время $t$, т.е. к неавтономным системам вида

$$
\dot{x}=\varepsilon f(x, t, \varepsilon) \text {. }
$$

В этом случае требование аналитичности по быстрой фазе является излишним: утверждение теоремы справедливо при $f$, непрерывной по $t$ и вешественно-аналитической по $x$. При этом замена переменных имеет вид

$$
x=y+\varepsilon U(y, t, \varepsilon),
$$

причем функция $U$ вешественно-аналитична по $y$, а гладкость по $t$ на единищу больше, чем гладкость $f$.

Для уравнения (4.4) отображение последования за время $t=2 \pi$ имеет вид $x \mapsto x_{1}$,

$$
x_{1}=x+\varepsilon \ell(x, \varepsilon) .
$$

Наоборот, любое отображение такого вида может быть представлено как отображение последования для некоторой системы вида (4.4) с непрерьвной (гладкой) по $t$ функцией $f(x, t, \varepsilon)$ (см., например, [20]).

ТеОРема 4.1.2 (А.И. Нейштадт [65]). Пусть функиия $\ell(x, \varepsilon)$ является вещественно-аналитической и удовлетворяет оченке $|\ell(x, \varepsilon)|<C$ при $x \in G+\delta$. Тогда при $y \in G+1 / 2 \delta, 0<\varepsilon<\varepsilon_{1}$ существует вещественно-аналитическая замена переменных

$$
x=y+\varepsilon U(y, \varepsilon), \quad|U|<c_{3},
$$

приводящая отображсение (4.5) к виду у $y_{1}$,

$$
y_{1}=y+\varepsilon(L(y, \varepsilon)+\alpha(y, \varepsilon)), \quad|\alpha|<c_{2} \exp \left(-c_{1}^{-1} / \varepsilon\right),
$$


причем укороченное отображсене (с отброшенным $\alpha$ ) включается в автономный поток, т.е. может быть представлено как отображение последования за время $t=2 \pi$ для автономной системь

$$
\dot{y}=\varepsilon F(y, \varepsilon), \quad|F-2 \pi \ell|<c_{4} \varepsilon .
$$

Постоянные $\varepsilon_{1}, c_{1}, c_{2}, c_{3}, c_{4}$ зависят от $\varepsilon_{0}, \delta, C$.

Если отображение - каноническое, то и замену можсно выбрать канонической, а систему - гамильтоновой; ее гамильтониан $H(x, \varepsilon)$ удовлетворяет оценке

$$
|H(x, \varepsilon)-E(x)|<c_{4} \varepsilon, \quad E(x)=2 \pi \int_{x_{0}}^{x}\left(\ell^{(q)} d p-\ell^{(p)} d q\right) .
$$

Здесь $p, q$ - сопряженные компоненты $x$, a $\ell^{(p)}, \ell^{(q)}$ - соответствующие им компоненты отображения $\ell$, взятые при $\varepsilon=0$, интеграл не зависит от пути в силу каноничности отображения.

Результаты А.И. Нейштадта уточнены в статье Д. В. Трешева [83].

4.2. Расщепление сепаратрис вблизи резонанса. Покажем, как теоремы из предыдущего пункта позволяют доказать экспоненциальную малость расщепления сепаратрис в окрестности простого резонанса системы с двумя степенями свободы.

Если в гамильтоновой системе с двумя степенями свободы, близкой к интегрируемой, выбрать одну из угловых переменных в качестве времени, то получится неавтономная гамильтонова система вида

$$
H=H_{0}(I)+\mu H_{1}(I, \varphi, t),
$$

где $I, \varphi$ - сопряженные переменные, $t$ - время, $\mu>0$ - мальй параметр. Гамильтониан $H$ периодичен по $\varphi$ и $t$ и аналитичен по всем переменным. Пусть $I_{*}-$ простое резонансное значение $I$ :

$$
\frac{\partial H_{0}}{\partial I}\left(I_{*}\right)=\frac{m}{n}, \quad \frac{\partial^{2} H_{0}}{\partial I^{2}}\left(I_{*}\right)=a \neq 0,
$$

где $m$ и $n$ - взаимно простые целые числа. Введем новые канонические переменные $p=\left(I-I_{*}\right) / \sqrt{\mu}, q=\varphi-m t / n$. Замена переменных не является канонической, однако в новых переменных уравнения движения все же имеют канонический вид с гамильтонианом

$$
E=\sqrt{\mu}\left(\frac{a p^{2}}{2}+V_{1}(q, t)\right)+\mu V_{2}(p, q, t, \mu), \quad V_{1}=H_{1}\left(I_{*}, \varphi, t\right) .
$$

Новый гамильтониан имеет период $2 \pi$ по $q$ и $2 \pi n$ по $t$. Если отбросить в (4.6) последний член и усреднить по $t$, то получится система с одной степенью свободы, описьвающая движение точки в периодическом потенциале $U(q)=\left\langle V_{1}(q, \cdot)\right\rangle($ с периодом $2 \pi / n)$. Пусть $U(q)$ имеет на каждом периоде единственную точку абсолютного максимума $q_{0}$, и притом невырожденную. Максимумам $U(q)$ отвечают неустойчивые положения равновесия, соединенные сепаратрисами. В полной системе при малых $\mu$ имеются неустойчивые периодические траектории, обладаюшие устойчивыми и неустойчивыми многообразиями. На одном периоде гамильтониана $E$ имеется $n$ абсолютных максимумов усредненного потенциала $U(q)$, соответствуюшие сепаратрисы образуют зону неустойчивости, ограничивающую "цепочку островов устойчивости".

Пусть $W^{s}$ и $W^{u}$ обозначают устойчивое и неустойчивое многообразия периодической траектории системы (4.6), лежащей в окрестности $q_{0}$. 
Теорема 4.2 .1 (А.И. Нейштадт [65]). Устойчивая и неустойчивая сепаратрисы системы (4.6) $W^{u}$ и $W^{s}$ лежсат в обвединении $K_{2} \exp \left(-K_{1}^{-1} / \sqrt{\mu}\right)$-окрестностей двух аналитических кривых, $2 \pi$-периодичных по q и однозначно проектирующихся на ось $q ; K_{1}$ и $K_{2}$ - положстельные постоянные.

СХемА ДОКАЗАТЕЛЬСТВА. Согласно теореме 4.1.1 существует замена переменных, переводящая (4.6) в автономную систему с одной степенњю свободы, на которую воздействует экспоненциально малое (порядка $O\left(\exp \left(-K_{4}^{-1} / \sqrt{\mu}\right)\right)$ периодическое по времени возмушение. Автономная система близка к системе, описываемой гамильтонианом $E_{0}=\sqrt{\mu}\left(\frac{a p^{2}}{2}+U(q)\right)$, и, следовательно, имеет устойчивую и неустойчивую сепаратрисы. Эти сепаратрисы могут быть представлены как объединение двух аналитических кривых. Теорема КАМ в форме [64], [51] гарантирует, что под воздействием возмушения порядка $O\left(\exp \left(-K_{4}^{-1} / \sqrt{\mu}\right)\right)$ в $K_{2} \exp \left(-K_{1}^{-1} / \sqrt{\mu}\right)$-окрестности невозмущенной сепаратрисы сохраняется часть инвариантных торов. Эти торы разделяют фазовое пространство и, следовательно, ограничивают инвариантную область, внутри которой лежат неустойчивые периодические траектории и их сепаратрисы.

Естественно, что оценки для величин, характеризуюших расщепление сепаратрис в окрестности их первого пересечения, таких, как гомоклинический инвариант, площадь луночки и некоторых других, могут быть получены без применения теории КАМ. Эти оценки получаются в силу непрерьвной (гладкой) зависимости локальных сепаратрис от параметра.

4.3. Квалифицированная оценка расщепления. Теорема 4.1.2 позволяет получить экспоненщиальные оценки расщепления сепаратрис при самых обших условиях. Однако она не дает ответа на вопрос о величине постоянной, стоящей в показателе экспоненты.

Следующая теорема устанавливает связь показателя экспоненты с расстоянием от вешественной оси до ближайшей особенности невозмущенной сепаратрисы в плоскости комплексного времени.

Рассмотрим систему в $\mathbb{R}^{2}$ вида

$$
\dot{p}=f(p)+\mu g(p, t / \varepsilon, \varepsilon) .
$$

Пусть $f: U \rightarrow \mathbb{R}^{2}, U \subset \mathbb{R}^{2}$, и $g: U \times \mathbb{S}^{1} \times\left[0, \varepsilon_{0}\right) \rightarrow \mathbb{R}^{2}$. Сделаем следуюшие предположения.

1. $\operatorname{div}(f+\mu g)=0$.

2. $g$ непрерьвна по совокупности аргументов, аналитична по $p$, периодична с периодом $T$ по второму аргументу и $\int_{0}^{T} g(p, s, \varepsilon) d s=0$.

3. Уравнение

$$
\dot{p}=f(p)
$$

имеет двоякоасимптотическую траекторию $\sigma(t)$, соединяющую седловые положения равновесия $p_{1}$ и $p_{2}$ и аналитичную в полосе $\Pi_{\delta_{0}}=\left\{t \in \mathbb{C}:|\operatorname{Im} t|<\delta_{0}\right\}$.

4. $f$ и $g$ аналитически продолжимы на окрестность $\sigma\left(\Pi_{\rho_{0}}\right)$ при некотором $0<\rho_{0}<\delta_{0}$. При этих условиях можно показать, что полная система (4.7) имеет гиперболические периодические траектории, близкие к $p_{1}$ и $p_{2}$ соответственно, при $|\mu| \leqslant \varepsilon^{p}, p>-1 / 2$, и всех достаточно малых $\varepsilon$. 
ТЕОРема 4.3 .1 (Э. Фонтик [22]). Пусть $p=\sigma\left(t_{0}\right)$ при некотором $t_{0} \in \mathbb{R} u$ $\eta \in(0, \delta)$. При перечисленных выше условиях $u|\mu| \leqslant \varepsilon^{p}, p>-1 / 2$, существуют постоянные $\varepsilon_{1}, C(\eta)>0$ такие, что при $\varepsilon \in\left(0, \varepsilon_{1}\right)$ расщепление сепаратрис $\boldsymbol{\theta}$ окрестности р на сечении $t=t_{0}$ ограничено сверху величиной вида

$$
C(\eta) \exp \left(-\frac{2 \pi \eta}{\varepsilon T}\right) \text {. }
$$

(В гетероклиническом случае необходимо также предположить существование главного семейства гетероклинической траектории полной системы.) Более того, если g не зависит от $p$, то тот же результат справедлив при р > -1.

Для доказательства рассмотрим отображение последования за период возмушения. В силу малости периода $\varepsilon T$ это отображение оказьвается близким к тождественному. Далее проверяется, что отображение последования удовлетворяет условиям теоремы 2.5.1, что немедленно влечет за собой все сфформулированные утверждения.

Эта теорема аналогична опубликованной ранее в работе [41]. Однако, насколько нам известно, до настоящего времени общедоступен только набросок соответствующего доказательства. В работе [41] налагаются несколько иные условия на правую часть уравнений: а) $f$ и $g$ предполагаются аналитическими функциями $p$ и $\varepsilon$; б) по $t$ предполагается гладкость класса $C^{1}$; в) $|\mu|<\varepsilon^{p}, p>0$; г) вместо условия нулевой дивергенщии система предполагается реверсивной по отношению к линейной обрашающей инволющии, при этом невозмущенная сепаратриса должна пересекать линию симметрии. Несложно видеть, что в приведенной выше теореме условия на гладкость несколько ослаблены.

Отметим, что по существу дела как условие нулевой дивергенции, так и условие обратимости нужно только для доказательства существования главного семейства двоякоасимптотических траекторий (хотя в цитированных здесь работах доказательства опираются на эти свойства более существенньм образом). Кроме того, неподвижная точка может быть параболической, однако в этом случае необходимо потребовать выполнения дополнительных условий, обеспечивающих сохранение параболических периодических траекторий и их сепаратрис под воздействием малого возмущения. Отметим, что один пример расшепления сепаратрис параболических точек описан в п. 3.3 .

\section{5. Метод Мельникова}

5.1. Функция Мельникова. Примеры. В настоящее время стандартным инструментом для исследования расшепления сепаратрис является метод Мельникова, называемый также методом Пуанкаре-Арнольда-Мельникова. Обычно этот метод применяют к системам, описываемым гамильтонианом вида

$$
H(x, y, t, \mu)=H_{0}(x, y)+\mu H_{1}(x, y, t)
$$

где $\mu$ является малым параметром. Предполагается, что при $\mu=0$ система имеет положение неустойчивого равновесия типа седла и гомоклиническую траекторию, подобно изображенной на рис. 8a). 

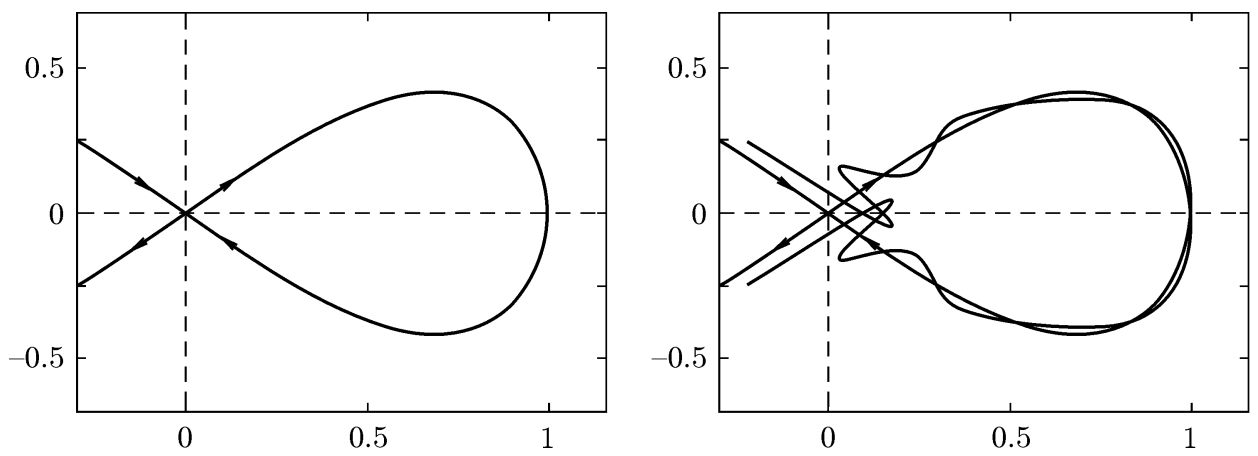

Рис. 8. а) Сепаратрисы невозмущенной системы с гамильтонианом $H_{0}=\frac{y^{2}}{2}-\frac{x^{2}}{2}\left(1-x^{2}\right)$.

б) Расщепление сепаратрис в сечении Пуанкаре плоскостью $t=0(\bmod 2 \pi)$ для $H=\frac{y^{2}}{2}-$ $\frac{x^{2}}{2}\left(1-x^{2}\right)+\mu x \cos \frac{t}{\varepsilon}, \mu=0.12, \varepsilon=0.25$

Тогда при достаточно малых значениях параметра возмушения полная система имеет периодическую гиперболическую траекторию, период которой совпадает с периодом по времени возмушения. Соответствуюшие устойчивые и неустойчивые многообразия близки к невозмущенной сепаратрисе, однако они могут расщепляться (рис. 8). Метод Мельникова основан на сравнении членов первого порядка в разложении устойчивой и неустойчивой сепаратрис в ряды по параметру возмущения $\mu$.

Более конкретно, пусть $\sigma_{0}(t)=\left(x_{0}(t), y_{0}(t)\right)$ обозначает гомоклиническую траекторию невозмушенной системы. В.И. Арнольд [4] заметил, что уравнения принимают особенно элегантньй вид, если в качестве одной из координат использовать невозмущенную энергию $H_{0}$. Тогда расстояние (разность значений $H_{0}$ ) между точками первого пересечения устойчивой и неустойчивой сепаратрис с нормалью к невозмушенной сепаратрисе, вьпушенной из точки $\sigma_{0}\left(t_{0}\right)$, равно $M\left(t_{0}\right)+O\left(\mu^{2}\right)$, где $M$ является функцией Мельникова:

$$
M\left(t_{0}\right)=\left.\int_{-\infty}^{\infty}\left\{H_{0}, H\right\}\right|_{\sigma_{0}(t), t+t_{0}} d t=\left.\mu \int_{-\infty}^{\infty}\left\{H_{0}, H_{1}\right\}\right|_{\sigma_{0}(t), t+t_{0}} d t
$$

здесь $\{f, g\}=\frac{\partial f}{\partial x} \frac{\partial g}{\partial y}-\frac{\partial f}{\partial y} \frac{\partial g}{\partial x}$ обозначает скобку Пуассона функций $f$ и $g$.

Несложно видеть, что в окрестности простых нулей функции Мельникова имеются трансверсальные гомоклинические траектории. Производная в нуле пропорциональна углу расщепления, а интеграл от функции Мельникова между двумя соседними нулями дает приближение для площади луночки.

Если гамильтониан $H$ зависит от дополнительного малого параметра $\varepsilon$, то функция Мельникова также может зависеть от этого параметра. В частности, если период возмущения пропорционален $\varepsilon$, то функция Мельникова оказьвается экспоненциально малой по $\varepsilon$. В этом случае приведенная вьше оценка погрешности оказьвается меньше функции Мельникова только для значений $\mu$, экспоненциально малых по сравнению c $\varepsilon$. Проиллюстрируем это на примерах. 
5.1.1. Пример (маятник). Популярной моделью для исследования экспоненциально малого расшепления сепаратрис является маятник с высокочастотным возмущением, описываемый гамильтонианом

$$
\frac{y^{2}}{2}+\cos x+\mu x \sin \frac{t}{\varepsilon} .
$$

Соответствующая функция Мельникова может быть легко вычислена:

$$
M\left(t_{0}\right)=\frac{2 \pi \mu}{\operatorname{ch} \frac{\pi}{2 \varepsilon}} \sin \frac{t_{0}}{\varepsilon} .
$$

При малых $\varepsilon$ амплитуда функции Мельникова имеет порядок $4 \pi \mu e^{-\pi / 2 \varepsilon}$. Эта величина хорошо согласуется с приведенньми ранее оценками сверху для амплитуды расщепления, имеющими для уравнения маятника вид $O\left(e^{-\rho / \varepsilon}\right)$ при любом $\rho<\pi / 2$.

Стандартная оценка погрешности аппроксимации для амплитуды расщепления сепаратрис имеет вид $O\left(\mu^{2}\right)$. Сравнивая эту величину с амплитудой функции Мельникова, видим, что функция Мельникова доминирует над погрешностью лишш при $|\mu| \ll e^{-\pi / \varepsilon}$. Ясно, что подобные значения $\mu$ нереалистично малы. С другой стороны, стандартные оценки не учитывают зависимость от дополнительного параметра, и можно надеяться, что постоянная в $O\left(\mu^{2}\right)$ убывает вместе с $\varepsilon$. На необходимость проведения соответствуюших оценок было указано в [73]. Начиная с работ [41], [75], была предпринята целая серия попыток доказать, что функция Мельникова доминирует над погрешностью при $|\mu|<\varepsilon^{p}$ для возможно меньшего значения $p$. Отметим, что в нашем примере гиперболическая периодическая траектория сохраняется вплоть до $p=-2$. В [41], [75] было использовано $p>8$, в работе [15] $-p>0$. В п. 5.3 мы приведем результаты, показьваюшие, что в данном примере функция Мельникова дает правильное предсказание для величины расщепления сепаратрис вплоть до $p>-1.5$, и вьпишем асимптотики расшепления, справедливые вплоть до $p=-2$.

Маятник с высокочастотньм возмушением стал моделью, на которой проводились испытания приложимости самых разнообразных методов к задаче об экспоненщиально малом расшеплении сепаратрис. Отметим среди них работы [19], [27], [3], [24], [29], [31], [84], [85]. Частичные результаты имеются также в [8], [47], [9], [6], [74].

5.1.2. Пример (мероморфная сепаратриса). Несложно найти (см., например, [22]) явное выражение для функции Мельникова для гамильтониана вида

$$
\frac{y^{2}}{2}+U(x)+\mu x G\left(\frac{t}{\varepsilon}\right)
$$

в предположении, что вторая компонента невозмущенного решения является мероморфной функцией. При этом амплитуда функции Мельникова имеет порядок $a \mu \varepsilon^{b} e^{-\rho / \varepsilon}$, где постоянная $a$ в обшем случае отлична от нуля. Легкость вычислений связана с возможностью применения теории вычетов при вычислении функции Мельникова, так как для подобного гамильтониана $\left\{H_{0}, H\right\}=\mu y G(t / \varepsilon)$.

Подобные системы являются естественными претендентами на обобщение результатов, полученных для уравнений маятника. Однако при оценке степени общности получаюшихся теорем необходимо проявлять осторожность. Например, из требований: 
“а) $y$-компонента сепаратрисы является мероморфной функцией, б) $U(x)$ является целой функцией”, вытекает, что $U(x)$ является либо тригонометрическим полиномом степени не вьше 2, либо алгебраическим полиномом степени не вьше 4 (ср. со следуюшим примером).

5.1.3. Пример (полиномиальньй потенщиал). Рассмотрим гамильтониан $H=$ $H_{0}+\mu H_{1}$, где

$$
H_{0}(x, y)=\frac{y^{2}}{2}-\frac{x^{2}}{2}\left(1-x^{n}\right), \quad H_{1}(x, y, t ; \varepsilon)=x \sin \frac{t}{\varepsilon}
$$

при $n \in \mathbb{N}$. Начало координат является седловой точкой невозмущенного гамильтониана. Соответствуюшая гомоклиническая траектория может быть найдена явно:

$$
x_{0}(t)=\frac{1}{\operatorname{ch}(n t / 2)^{2 / n}}, \quad y_{0}(t)=-\frac{\operatorname{sh}(n t / 2)}{\operatorname{ch}(n t / 2)^{(n+2) / n}},
$$

она является аналитической функцией и имеет особенности в точках $t=i \frac{\pi k}{n}$, $k \in \mathbb{Z}$. Эти особенности являются полюсами второго и первого порядка при $n=1$ и $n=2$ соответственно, а при всех остальных значениях $n$ особенности являются рациональньми точками ветвления.

Полная система имеет при не слишком больших $\mu$ периодическую траекторию с периодом $2 \pi \varepsilon$ в окрестности невозмушенного седла. Соответствуюшие инвариантные многообразия вьглядят как на рис. 8. Функция Мельникова имеет вид

$$
M\left(t_{0}\right)=\mu \int_{-\infty}^{\infty} y_{0}(t) \sin \frac{t+t_{0}}{\varepsilon} d t
$$

Этот интеграл можно оценить следуюшим образом:

$$
M\left(t_{0}\right)=\frac{2 \pi \mu}{\Gamma(2 / n)}\left(\frac{n}{2}\right)^{2 / n} \varepsilon^{1-2 / n} e^{-\pi / n \varepsilon} \cos \frac{t_{0}}{\varepsilon}(1+O(\varepsilon)) .
$$

При малых $\varepsilon$ амплитуда функции Мельникова является величиной порядка const $\mu \varepsilon^{1-2 / n} e^{-\pi / n \varepsilon}$. Последнее выражение больше, чем погрешность в стандартной теории, $O\left(\mu^{2}\right)$, при условии $|\mu| \ll \varepsilon^{1-2 / n} e^{-\pi / n \varepsilon}$. Это условие малости амплитуды возмушения также может быть существенным образом ослаблено.

5.1.4. Пример (метод Мельникова дает точное значение амплитуды расщепления). Обычно метод Мельникова дает лишш приближенное значение для амплитуды и других величин, характеризуюших расшепление сепаратрис. В следуюшем примере, предложенном К. Симо и Э. Фонтиком [78], метод Мельникова дает точное значение для величины расщепления. Рассмотрим систему с гамильтонианом

$$
H=\left(1-x^{2}\right) y+\mu x \sin \frac{t}{\varepsilon} .
$$

Соответствуюшие уравнения движения имеют вид

$$
\dot{x}=1-x^{2}, \quad \dot{y}=2 x y-\mu \sin \frac{t}{\varepsilon} .
$$


Переменные разделяются, и траектории могут быть выписаны явно. Вычислим функцию Мельникова. При $\mu=0$ система имеет гетероклиническую траекторию

$$
x_{0}(t)=\text { th } t, \quad y_{0}(t)=0
$$

соединяющую две седловые точки $(-1,0)$ и $(1,0)$. После несложных вычислений получаем интеграл Мельникова:

$$
M\left(t_{0}\right)=\mu \int_{-\infty}^{\infty}\left(1-x_{0}^{2}\right) \sin \frac{t+t_{0}}{\varepsilon} d t=\mu \int_{-\infty}^{\infty} \frac{\sin \frac{t+t_{0}}{\varepsilon}}{\operatorname{ch}^{2} t} d t .
$$

Вычисляя его по вычетам, имеем

$$
M\left(t_{0}\right)=\frac{\pi \mu}{\varepsilon \operatorname{sh} \frac{\pi}{2 \varepsilon}} \sin \frac{t_{0}}{\varepsilon}
$$

Несложно проверить, что функция Мельникова дает точное значение для расщепления сепаратрис. Дело в том, что возмущение не затрагивает первую компоненту движения, а только эта компонента входит в выражение для

$$
\dot{H}_{0}=\mu\left\{H_{0}, H_{1}\right\}=\left(1-x^{2}\right) \sin \frac{t}{\varepsilon} .
$$

5.1.5. Пример (любопытное обобщение предыдущего). Во всех предыдущих примерах при малых $\varepsilon$ расщепление сепаратрис вело себя как const $\mu \varepsilon^{a} e^{\rho_{0} / \varepsilon}$, где $\rho_{0}$ является ближайшей к вешественной оси особенностью невозмушенной сепаратрисы. В приводимом ниже примере эта особенность расположена в точке $i \pi / 2$. Соответственно согласно общей теории расщепление сепаратрис допускает оценку сверху вида $O\left(e^{-(\pi-\delta) / 2 \varepsilon}\right)$. Было установлено [76], что расшепление сепаратрис не допускает оценки вида $O\left(\varepsilon^{-N} e^{-\pi / 2 \varepsilon}\right)$ ни при каком $N$.

Опишем соответствуюшую систему. Рассмотрим плоскость, снабженную симплектической формой

$$
\Omega=e^{\alpha x+\beta x^{2}} d x \wedge d y
$$

где $\alpha$ и $\beta$ являются вещественными параметрами. Этой симплектической форме соответствует скобка Пуассона

$$
\{f, g\}=e^{-\alpha x-\beta x^{2}}\left(\frac{\partial f}{\partial x} \frac{\partial g}{\partial y}-\frac{\partial f}{\partial y} \frac{\partial g}{\partial x}\right)
$$

Рассмотрим на этой плоскости систему, описываемую гамильтонианом

$$
H(x, y, t)=H_{0}(x, y)+\mu H_{1}(x, t / \varepsilon)
$$

где

$$
H_{0}(x, y)=e^{\alpha x+\beta x^{2}}\left(1-x^{2}\right) y, \quad H_{1}(x, \tau)=\cos \tau \int_{0}^{x} e^{\alpha x+\beta x^{2}} d x
$$


При выводе уравнений Гамильтона необходимо учитьвать вид симплектической формы. Получаем систему

$$
\dot{x}=e^{-\alpha x-\beta x^{2}} \frac{\partial H}{\partial y}, \quad \dot{y}=-e^{-\alpha x-\beta x^{2}} \frac{\partial H}{\partial x},
$$

при подстановке функции Гамильтона $H$ получается

$$
\begin{aligned}
& \dot{x}=1-x^{2}, \\
& \dot{y}=\left(2 x-(\alpha+2 \beta x)\left(1-x^{2}\right)\right) y+\mu \cos \frac{t}{\varepsilon} .
\end{aligned}
$$

Отметим, что правая часть является полиномиальной. Как и в предыдущем примере, уравнения движения разделяются и траектории могут быть найдены явно. При $\mu=0$ система имеет гетероклиническую траекторию (5.3). Выпишем функцию Мельникова

$$
\begin{aligned}
M\left(t_{0}\right) & =\left.\mu \int_{-\infty}^{\infty}\left\{H_{0}, H_{1}\right\}\right|_{x_{0}(t), y_{0}(t), t+t_{0}} d t \\
& =\mu \int_{-\infty}^{\infty} e^{-\alpha x_{0}(t)-\beta x_{0}^{2}(t)}\left(1-x_{0}^{2}(t)\right) \cos \frac{t+t_{0}}{\varepsilon} d t .
\end{aligned}
$$

Несмотря на то, что правая часть уравнений Гамильтона полиномиальна, подынтегральная функция имеет сушественную особенность. Пусть $\alpha=0$. Тогда, учитьвая нечетность $x_{0}$, имеем

$$
M\left(t_{0}\right)=\cos \frac{t_{0}}{\varepsilon} \mu \int_{-\infty}^{\infty} e^{-\beta x_{0}^{2}(t)}\left(1-x_{0}^{2}(t)\right) e^{i t / \varepsilon} d t .
$$

Учитьвая явную формулу для $x_{0}$, получаем выражение для амплитуды функции Мельникова:

$$
\mu \int_{-\infty}^{\infty} \frac{e^{-\beta \operatorname{th}^{2} t+i t / \varepsilon}}{\operatorname{ch}^{2} t} d t
$$

Асимптотика последнего интеграла может быть найдена по методу перевала и при $\beta=-\varepsilon$ имеет вид

$$
c_{1} \varepsilon^{a} \exp \left(-\frac{\pi}{2 \varepsilon}+\frac{c_{2}}{\varepsilon^{1 / 3}}\right),
$$

где $c_{1}$ и $c_{2}-$ подходяшие положительные постоянные. Последняя оценка согласуется с теоремой 1 работы [76], т.е. расшепление сепаратрис действительно не допускает оценки сверху вида $O\left(\varepsilon^{-N} e^{-\pi / 2 \varepsilon}\right)$; однако противоречит замечанию 2 [76; с. 194], т.е. расшепление сепаратрис не имеет вида произведения сходяшегося ряда Лорана на $e^{-\pi / 2 \varepsilon}$.

5.1.6. Пример (гетероклиническая связка может исчезнуть). Если невозмущенная траектория является гетероклинической, то можно построить такое возмущение, что возмушенная система не будет иметь гетероклинических траекторий. Однако при этом функция Мельникова будет экспоненциально малой и будет иметь простые нули. Проиллюстрируем это следуюшим примером. Рассмотрим гамильтониан $H=$ $H_{0}+\mu H_{1}$,

$$
H_{0}=\frac{y^{2}}{2}+\cos x+r \cos 2 x, \quad H_{1}=\cos \left(x-\frac{\pi}{4}\right) \sin \frac{t}{\varepsilon} .
$$


При $r<-1 / 4$ невозмущенная система имеет гетероклиническую траекторию, которая соединяет две седловые точки $( \pm \arccos (-1 /(4 r)), 0)$ (см. рис. 4). Несложно найти соответствуюшую функцию Мельникова и убедиться, что она имеет простые нули, в то время как ее амплитуда имеет порядок $C \mu \varepsilon^{-1} e^{-\rho / \varepsilon}$. Таким образом, наивное применение метода Мельникова предсказьвает наличие гетероклинических траекторий и экспоненщиальную малость расщепления.

С другой стороны, невозмущенную гетероклиническую траекторию можно уничтожить произвольно малым автономньм возмущением, добавляя к гамильтониану функцию, значения которой в рассматриваемых двух седловых точках неодинаковы. В результате у возмущенной системы седла оказываются на различных линиях постоянной энергии, и их сепаратрисы не могут пересекаться. Аналогичное явление наблюдается в нашем примере. Метод усреднений позволяет построить производящую функцию вида

$$
S\left(x, Y, \frac{t}{\varepsilon} ; \varepsilon, \mu\right)=x Y+\varepsilon s_{1}\left(x, Y, \frac{t}{\varepsilon}, \mu\right)+\varepsilon^{2} s_{2}\left(x, Y, \frac{t}{\varepsilon}, \mu\right)+\varepsilon^{3} s_{3}\left(x, Y, \frac{t}{\varepsilon}, \mu\right),
$$

с помошю которой зависимость от времени уничтожается с точностью до $O\left(\varepsilon^{3}\right)$. В новых переменных гамильтониан принимает вид

$$
\widehat{H}_{0}(X, Y ; \varepsilon, \mu)+O\left(\varepsilon^{3}\right),
$$

где

$$
\widehat{H}_{0}(X, Y ; \varepsilon, \mu)=\frac{y^{2}}{2}+\cos x+r \cos 2 x+\frac{\mu^{2} \varepsilon^{2}}{8}(1+\sin 2 x) .
$$

Новый невозмушенный гамильтониан имеет две седловые точки, близкие к невозмущенным. Однако они лежат на разных уровнях $\widehat{H}_{0}$ и, следовательно, не могут быть соединенными гетероклинической траекторией (см. рис. 4). Продолжая применять метод усреднений, можно показать, что возмущение порядка $O\left(\varepsilon^{3}\right)$ не может привести к возникновению гетероклинических траекторий.

Таким образом, исходная гамильтонова система не имеет гетероклинических траекторий при всех достаточно малых положительных $\varepsilon$, в то время как метод Мельникова предсказывает наличие гетероклинических траекторий с экспоненциально мальм расщеплением.

5.2. Случай возмущения малой амплитуды. В настоящем разделе мы рассмотрим малые высокочастотные возмушения гамильтоновых систем на плоскости

$$
H\left(x, y, \frac{t}{\varepsilon} ; \varepsilon, \mu\right)=H_{0}(x, y)+\mu H_{1}\left(x, y, \frac{t}{\varepsilon} ; \varepsilon, \mu\right),
$$

где $|\mu|<\mu_{0} \varepsilon^{p}$, а $\mu_{0}$ и $p$ являются подходяшими постоянньми. Мы установим достаточные условия, при которых метод Мельникова правильно предсказывает расщепление сепаратрис для гамильтониана этого вида. Отметим, что расщепление сепаратрис в этом случае экспоненщиально мало. Тот факт, что применение метода Мельникова к подобным системам требует специального обоснования, был отмечен еще в работе [73].

Сформулируем условия, которые мы наложим на исследуемую систему. 
Предположим, что гамильтониан (5.5) вещественно-аналитичен по $x$ и $y$ и $2 \pi$-периодичен по своему третьему аргументу. Несложно показать, что существует такая постоянная $\rho$, что невозмушенная сепаратриса $\sigma_{0}(t)$ аналитична в полосе $\Pi_{\rho}=\{t \in \mathbb{C}$ : $|\operatorname{Im} t|<\rho\}$ и не является аналитической ни в какой более широкой полосе $\Pi_{\rho^{\prime}}\left(\rho^{\prime}>\rho\right)$.

[H1] Невозмущенная система, описываемая гамильтонианом $H_{0}$, имеет две седловые точки, соединенные гетероклинической траекторией $\sigma_{0}(t)$. Мы не исключаем гомоклинический случай, когда эти две седловые точки совпадают.

[H2] $H_{0}$ и $H_{1}$ являются вешественно-аналитическими функциями $x$ и $y$, определенньми на открытом подмножестве $\mathbb{C}^{2}$, содержащем $\sigma_{0}\left(\Pi_{\rho}\right)$.

[H3] $H_{1}$ непрерывна по совокупности аргументов и имеет нулевое среднее значение: $\int_{0}^{2 \pi} H_{1}(x, y, s ; \varepsilon, \mu) d s=0$.

[H4] Для некоторых значений постоянных $\mu_{0}$ и $p$ выполнено свойство продолжимости при $|\mu|<\mu_{0} \varepsilon^{p}$.

ОПРЕДЕЛЕНИЕ 5.2.1 (свойство продолЖимости). Будем говорить, что система, описываемая гамильтонианом (5.5), обладает свойством продолжимости при $|\mu|<\mu_{0} \varepsilon^{p}$, если существуют такие постоянные $q_{1}, q_{2}<p+1$ u $r>0$, что по любым постоянным $C>0, R>0$ можно найти такие $C_{1}, C_{2}$, что любая траектория $p(t)$ с начальными условиями вблизи невозмущенной сепаратрисы:

$$
\left\|p\left(t_{0}\right)-\sigma_{0}\left(t_{1}\right)\right\|<C \mu \varepsilon
$$

$t_{0} \in \mathbb{R}, \operatorname{Re} t_{1}<-R,\left|\operatorname{Im} t_{1}\right|<\rho-r \varepsilon$, остается близкой $\kappa$ невозмущенной сепаpampuce npu вcex $t \in[0,2 R]$ :

$$
\left\|p\left(t_{0}+t\right)-\sigma_{0}\left(t_{1}+t\right)\right\|<C_{1}|\mu| \varepsilon^{1-q_{1}},
$$

и, кроме того, справедлива следующая оценка:

$$
\int_{0}^{t}\left|\left\{H_{0}, H_{1}\right\}\right|_{p\left(t_{0}+\tau\right), \tau / \varepsilon}-\left.\left\{H_{0}, H_{1}\right\}\right|_{\sigma_{0}\left(t_{1}+\tau\right), \tau / \varepsilon}\left|d \tau<C_{2}\right| \mu \mid \varepsilon^{1-q_{2}} .
$$

Для всех известных примеров вторая оценка может быть выведена из первой. Однако при этом получаются неоптимальные оценки для постоянной $q_{2}$.

ОПРЕДЕЛЕНИЕ 5.2.2. Главная гетероклиническая (гомоклиническая) траектория является членом семейства $\gamma_{\varepsilon}$ гетероклинических (гомоклинических) траекторий, которое равномерно близко к невозмущенной сепаратрисе.

[Н5] Алгебраическая величина площади множества, ограниченного отрезками инвариантных многообразий, заключенными между двумя последовательными пересечениями главной гомоклинической траектории с плоскостью $t=0$ $(\bmod 2 \pi)$, равна нулю.

Последнее свойство означает, что площади луночек равны (см. рис. 8). В гомоклиническом случае существование главной гомоклинической траектории, так же как и условие [Н5], вытекает из того, что отображение последования за период возмушения сохраняет площадь. 
ТеОРема 5.2.1 [30]. Пусть $q=\min \left\{2-2 q_{1}, 1-q_{2}\right\}$ и выполнены условия [H1]-[Н5]. Тогда справедливо следующее. Существует $\varepsilon_{0}>0$ такое, что при всех $\varepsilon \in$ $\left(0, \varepsilon_{0}\right)$ в окрестности сегмента локального инвариантного многообразия $W_{\mathrm{loc}}^{s}$ поток может быть выпрямлен с помощью канонической $2 \pi \varepsilon$-периодической по времени замены координат $(x, y, t) \mapsto(T, E, t)$. В новых координатах гамильтониан совпадает с переменной $E, W_{\mathrm{loc}}^{s}$ задается уравнением $E=0$, а неустойчивое многообразие, когда оно в первый раз попадает в область определения системы координат, задается графиком $E=\Theta(T-t)$, где $\Theta$ является $2 \pi \varepsilon-n е-$ риодической функцией (см. рис. 4),

$$
\Theta\left(t_{0}\right)=M\left(t_{0}\right)+O\left(\mu^{2} \varepsilon^{q} e^{-\rho / \varepsilon}\right), \quad t_{0} \in \mathbb{R} .
$$

Производные этой замены по х и у ограничены равномерно по $\varepsilon, 0<\varepsilon<\varepsilon_{0}$.

Иначе говоря, условия [Н1]-[Н5] являются достаточными условиями для применимости метода Мельникова к исследованию расшепления сепаратрис системы (5.5).

Во многих примерах функция Мельникова оказывается больше оценки погрешности, устанавливаемой этой теоремой при условии $\mu=\mu_{0} \varepsilon^{p}$, где $p$ не слишком мало. Только в этом случае теорема дает нижнюю гранищу для расшепления сепаратрис. В случае же, когда функция Мельникова оказывается меньше погрешности, теорема 5.2.1 дает более тонкую верхнюю оценку для расшепления по сравнению с общим случаем.

Эта теорема применима к примерам из предыдушего пункта. Оценки из работы [15] могут быть приспособлены для того, чтобы проверить, что гамильтониан (5.1) удовлетворяет свойству продолжимости при $|\mu|<\mu_{0} \varepsilon^{p}$ с $p>2 / n$ и $q_{1}=q_{2}=1+2 / n$. Тогда $q=-2 / n$ и, сравнивая с $(5.2)$, получаем, что амплитуда функции Мельникова больше погрешности из (5.8) при условии, что $\mu \varepsilon^{1-2 / n} \gg \mu^{2} \varepsilon^{-2 / n}$. Следовательно, метод Мельникова правильно предсказывает величину расщепления для $|\mu|<\mu_{0} \varepsilon^{p}$ при $p>\max \{1,2 / n\}$.

Условия [H1]-[Н3] и [Н5] позволяют получить верхнюю оценку для расщепления сепаратрис вида $O\left(e^{-\rho_{1} / \varepsilon}\right), \rho_{1}=\rho-\delta$, где $\delta$ является произвольной положительной постоянной. Оценки типа (5.6) и (5.7) вьполнены, если траектория не проходит вблизи особенности невозмущенной сепаратрисы. Следовательно, условие [H4] накладьвает ограничения на поведение траекторий системы в окрестности особенности. В частности, эта гипотеза справедлива, когда $H_{0}$ и $H_{1}$ полиномиальны по $x$ и $y$, а все особенности являются либо логарифмическими, либо полюсами, либо рациональными. При это полиномы могут быть как алгебраическими, так и тригонометрическими. Таким образом, метод Мельникова, вообе говоря, применим для произвольного полиномиального гамильтониана при $|\mu|<\mu_{0} \varepsilon^{p}$, однако нахождение оптимального значения $p$ требует детального исследования динамики в окрестности невозмущенной сепаратрисы.

Отметим, что частные случаи приведенной выше теоремы были рассмотрены в работах А. Дельзамса и Т. Сеары (соответствующие ссылки см. в [16]).

5.3. Маятник с большим высокочастотным возмущением. Нахождение оценки снизу для расщепления сепаратрис в системах с высокочастотным возмущением является непростой задачей. Стандартньм методом здесь является метод Мельникова, оправдание применимости которого к широкому классу гамильтоновых систем 
можно найти в предыдущем пункте. Одним из важнейших условий для этого является малость амплитуды возмушения. В случае, когда амплитуда возмушения не мала, метод Мельникова, вообще говоря, не применим.

Приведем соответствуюшие результаты для ряда примеров, носяших полиномиальньй характер. На этих примерах мы установим границы применимости метода Мельникова, а также выпишем асимптотические формулы, верные даже тогда, когда метод Мельникова не применим. Эти формулы отличаются от полученных по методу Мельникова множителем, значение которого может быть найдено при решении некоторой вспомогательной задачи.

Первая задача о возмущении маятника. Наиболее популярной моделью для исследования расшепления сепаратрис стал маятник, описываемый гамильтонианом

$$
\frac{y^{2}}{2}+\cos x-a \varepsilon^{-2} x \sin \frac{t}{\varepsilon} .
$$

Амплитуда возмушения неограниченно растет при малых $\varepsilon$, однако этот рост может быть скомпенсирован заменой переменных

$$
\left(\begin{array}{l}
x \\
y
\end{array}\right) \mapsto\left(\begin{array}{c}
x+a \sin \frac{t}{\varepsilon} \\
y+a \varepsilon^{-1} \cos \frac{t}{\varepsilon}
\end{array}\right) .
$$

Эта замена, эквивалентная двум шагам классического метода усреднений, заимствована нами у К. Симо. Обозначим, надеясь, что это не вызовет недоразумения, новые переменные через $x$ и $y$. При этом гамильтониан принимает вид

$$
H(x, y, t, a, \varepsilon)=\frac{y^{2}}{2}+\cos \left(x-a \sin \frac{t}{\varepsilon}\right),
$$

его среднее значение за период по времени равно

$$
H_{0}(x, y, a)=\frac{y^{2}}{2}+J_{0}(a) \cos x
$$

где $J_{0}$ обозначает функцию Бесселя нулевого порядка.

Для плошади луночки (см. рис. 9) имеет место асимптотическая формула

$$
\mathscr{A}=8 \pi a \varepsilon^{-1} \exp \left(-\frac{\pi}{2 \varepsilon \sqrt{\left|J_{0}(a)\right|}}\right) \cdot\left(f_{1}(a)+O(\varepsilon)\right) .
$$

Это результат совпадает с предсказанием метода Мельникова, примененного к преобразованному гамильтониану, за исключением корректирующего множителя $f_{1}(a)$. Функция $f_{1}(a)$ является вешественно-аналитической функцией, определенной на всей вешественной оси, из которой удалены точки $a=j_{0 k}$, являющиеся нулями функции Бесселя $J_{0}$. Кроме того, $f_{1}(a)=1+O\left(a^{2}\right)$ при малых $a$. График функции $f_{1}(a)$ приведен на рис. 10.

Множитель $f_{1}$ возникает при исследовании комплексных инвариантных многообразий системы

$$
\frac{v^{2}}{2}-e^{u} e^{-i a \sin \tau}
$$




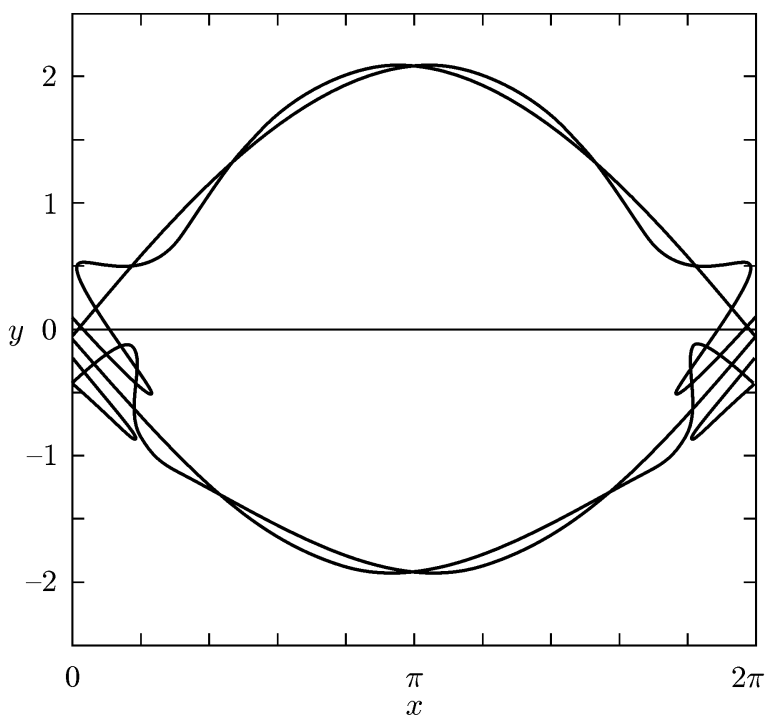

Рис. 9. Сепаратрисы отображения последования для периодически возмущенного маятника

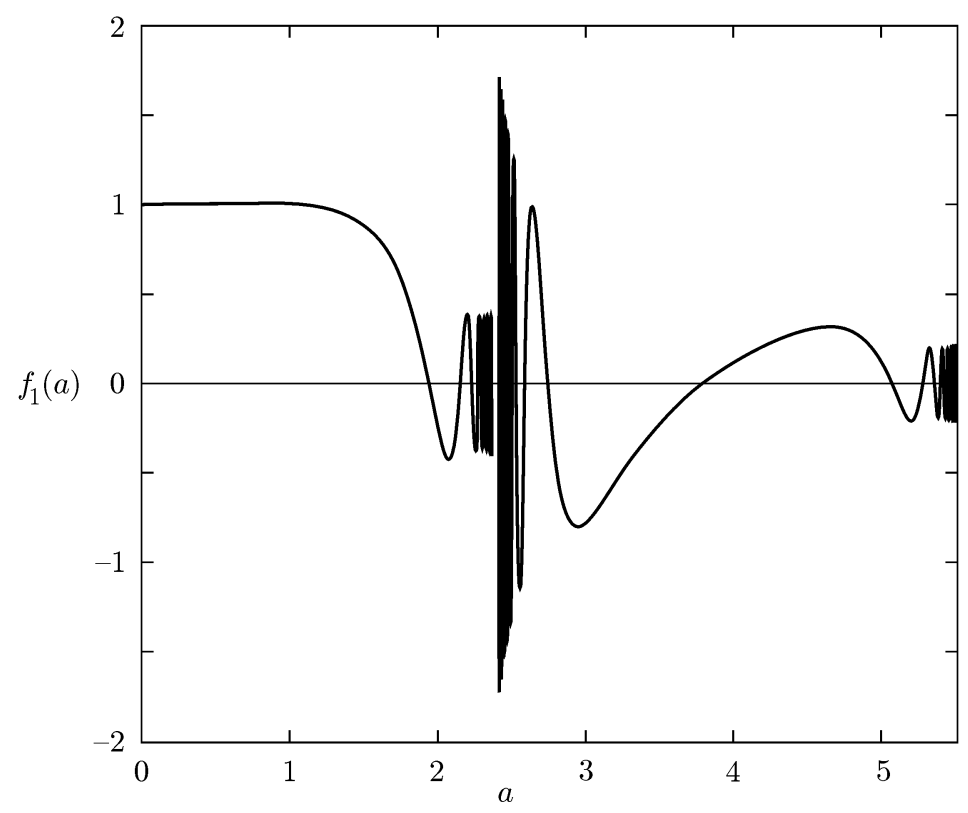

Рис. 10. Корректирующий множитель для системы $H\left(x, y, \frac{t}{\varepsilon}, a\right)=\frac{y^{2}}{2}+\cos \left(x-a \sin \frac{t}{\varepsilon}\right)$ как функция параметра $a$ при $0 \leqslant a \leqslant j_{02}$

Будем назьвать подобные системы модельнымми.

Отметим, что при $j_{01}<a<j_{02}$ функция Бесселя $J_{0}(a)$ отрицательна. Соответственно сепаратриса невозмушенной системы ассоциирована с седловой точкой $(-\pi, 0)$. Полная система имеет в окрестности этого седла периодическую траекторию, и ее 
инвариантные многообразия близки к невозмущенной сепаратрисе. Они похожи на рис. 9, однако сдвинуты по горизонтали на $\pi$. При $j_{02}<a<j_{03}$ седловая точка невозмущенной системы снова оказьвается в начале координат. Ситуация повторяется каждьй раз, когда $а$ проходит через нуль функции Бесселя $J_{0}$. Асимптотическая формула остается справедливой для всех интервалов между нулями функции Бесселя. Корректируюший множитель определяется из одной и той же модельной системы.

На основе численных исследований можно выдвинуть гипотезу о том, что функция $f_{1}(a)$ имеет бесконечное число нулей $a_{n k}$ на каждом из интервалов $\left(j_{0, k}, j_{0, k+1}\right)$, нули корректируюшего множителя накапливаются к концам интервалов: $a_{n k} \approx j_{0, k+1}-$ $c_{k+1}^{-} / n$ при $n \rightarrow \infty$ и $a_{n k} \approx j_{0, k}+c_{k}^{+} / n$ при $n \rightarrow-\infty$. Таким образом, с каждым нулем $j_{0 k}$ можно связать два числа $c_{k}^{-}$и $c_{k}^{+}$, которые описьвают скорость накопления нулей слева и справа. С помошю компютера мы получили для $j_{01}$ значения $c_{1}^{-}=0.41$ и $c_{1}^{+}=0.42$. Амплитуда колебаний $f_{1}(a)$, по-видимому, стремится к пределу по мере того, как $a$ приближается к $j_{0 k}$. Левый и правьй пределы при этом не совпадают. Аналитическое доказательство последних утверждений отсутствует на момент написания настоящей работы.

Маятник с колеблющейся точкой подвеса. В конце прошлого века А. Пуанкаре [69] открыл явление расщепления сепаратрис. При этом в качестве примера он исследовал систему, которая может быть описана гамильтонианом

$$
\frac{y^{2}}{2}+\cos x+a \sin x \cos \frac{t}{\varepsilon} .
$$

Эта система отличается от предыдущей по сути дела только спектром возмущения. Для расщепления сепаратрис справедлива асимптотическая формула, аналогичная описанной выше. А именно, плошадь луночки

$$
\mathscr{A}=8 \pi a \varepsilon^{-1} \exp \left(-\frac{\pi}{2 \varepsilon}\right) \cdot\left(f_{2}(a)+O(\varepsilon)\right)
$$

где $f_{2}(a)$ является четной целой функцией одной комплексной переменной, график которой приведен на рис. 11.

Модельная система в этом случае имеет вид

$$
\frac{v^{2}}{2}-e^{u}(1-i a \cos \tau)
$$

Эта модельная система описывает корректируюший множитель для расшепления сепаратрис более общего возмушения маятника, а именно для

$$
\frac{y^{2}}{2}+\cos x+a_{1} \cos \left(x+\phi_{1}-\frac{t}{\varepsilon}\right)+a_{2} \cos \left(x+\phi_{2}+\frac{t}{\varepsilon}\right) .
$$

Если $a_{2} \neq 0$, то корректирующий множитель равен $\left|f_{2}(a)\right|$, где $a^{2}=-4 a_{1} a_{2} e^{i\left(\phi_{1}+\phi_{2}\right)}$. Площадь луночки равна

$$
\mathscr{A}=8 \pi \varepsilon^{-1} \exp \left(-\frac{\pi}{2 \varepsilon}\right) \cdot\left(2 a_{2}\left|f_{2}(a)\right|+O(\varepsilon)\right) .
$$



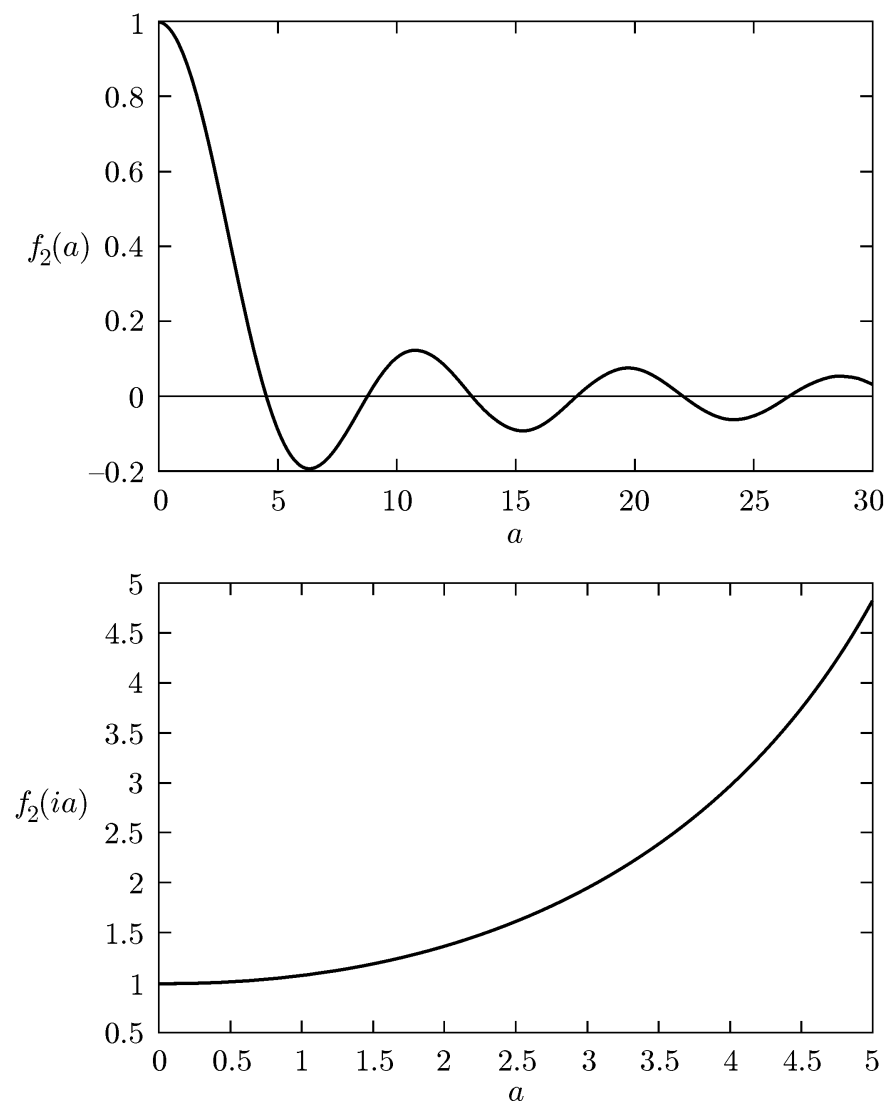

Рис. 11. Корректирующий множитель как функция от параметра $a$ для:

a) $H(x, y, t, a, \varepsilon)=\frac{y^{2}}{2}+\cos x+a \sin x \cdot \sin \frac{t}{\varepsilon}$;

б) $H(x, y, t, a, \varepsilon)=\frac{y^{2}}{2}+\cos x+a \cos x \cdot \sin \frac{t}{\varepsilon}$

Д. В. Трещев [84] получил независимо аналогичную формулу, используя метод усреднения с непрерьвным параметром. Предложенньй им метод позволяет вычислить несколько первых коэффициентов ряда Тейлора для корректирующего множителя.

Модель, предложенная Б. В. Чириковьм для исследования взаимодействия нелинейных резонансов (см., например, [12]), является частным случаем рассмотренной вьше системы. Она соответствует выбору $a_{1}=0$, следовательно, корректирующий множитель в точности равен 1 . То есть в этом частном случае метод Мельникова правильно предсказьвает величину расщепления даже для возмущений конечной амплитуды, в то время как для более обшего случая он применим только для малых амплитуд.

В комплексной плоскости переменной $а$ корректирующий множитель ведет себя качественно как $\left|\frac{\sin (a / \sqrt{2})}{a / \sqrt{2}}\right|$ (наблюдение К. Симо). В частности, на рис. 11 б) приведен 
график корректирующего множителя на чисто мнимой оси переменной $a$. Это соответствует случаю $a_{1}, a_{2} \neq 0$ и $\phi_{1}+\phi_{2}=0$.

Возмущенное уравнение Дуффинга. Еще одной популярной моделью является уравнение Дуффинга, высокочастотное возмушение которого описывается гамильтонианом

$$
\frac{y^{2}}{2}-\frac{x^{2}}{2}+\frac{x^{4}}{4}-x a \varepsilon^{-2} \sin \frac{t}{\varepsilon} .
$$

Применим метод Мельникова к гамильтониану, получающемуся после выполнения двух шагов метода усреднений. Преобразованный гамильтониан имеет вид

$$
H_{0}+H_{1}
$$

где

$$
\begin{aligned}
& H_{0}=\frac{y^{2}}{2}-\frac{x^{2}}{2}\left(1-\frac{3 a^{2}}{2}\right)+\frac{x^{4}}{4} \\
& H_{1}=-x^{3} a \sin \frac{t}{\varepsilon}-x^{2} \frac{3 a^{2}}{4} \cos \frac{2 t}{\varepsilon}+x a \sin \frac{t}{\varepsilon}\left(1-a^{2} \sin ^{2} \frac{t}{\varepsilon}\right) .
\end{aligned}
$$

Имеет место асимптотическая формула

$$
\mathscr{A}=\frac{4 \pi \sqrt{2} a}{\varepsilon^{3}\left(1-\frac{3}{2} a^{2}\right)^{3}} \exp \left(-\frac{\pi}{2 \varepsilon \sqrt{1-\frac{3}{2} a^{2}}}\right)(1+O(\sqrt{\varepsilon})) \text {. }
$$

Корректируюший множитель для данной задачи равен единице, так как соответствующая модельная система

$$
\frac{v^{2}}{2}+\frac{u^{4}}{4}-u^{3} \widetilde{a} \sin \tau
$$

$\widetilde{a}=\varepsilon a$, может быть рассмотрена как регулярная задача теории возмущений, и для ее исследования может быть применен метод Мельникова.

5.4. Схема доказательства. Опишем теперь схематически вьвод асимптотических формул, приведенных в предыдущем пункте, и объясним, какую роль в этом вьводе играют модельные системы. Подробнее доказательство асимптотической формулы в случае первого возмущения маятника приведено в [29].

Пусть гамильтониан $H(x, y, t / \varepsilon)$ непрерьвен по всем переменным и аналитичен по пространственньм. Пусть $H_{0}(x, y)$ - его среднее значение за период по времени - имеет седловую точку, которой соответствует гомоклиническая траектория $\left(x_{0}, y_{0}\right)(t)$, аналитичная в полосе $|\operatorname{Im} t|<\rho$ и имеющая ровно по одной особой точке на верхней и нижней границах этой полосы. Сдвигом по времени можно добиться того, чтобы эти особенности располагались в точках $\pm i \rho$.

Тогда полная система имеет гиперболическую периодическую траекторию в окрестности невозмушенного седла. Локальные инвариантные многообразия этой траектории близки с невозмущенной сепаратрисе. Как было показано ранее, расшепление сепаратрис не превосходит $O\left(\exp \left(-\frac{\rho-\delta}{\varepsilon}\right)\right)$. 
Шаг 1. Параметризашия сепаратрис. В расширенном фазовом пространстве сепаратрисы полной системы могут быть представлены в параметрическом виде

$$
x=x^{u, s}\left(t_{1}, t_{2} ; \varepsilon\right), \quad y=y^{u, s}\left(t_{1}, t_{2} ; \varepsilon\right), \quad t=t_{2},
$$

параметризуюшие функции периодичны по $t_{2}$ с периодом $2 \pi \varepsilon$, кроме того, при подстановке

$$
t_{1}=t+t_{0}, \quad t_{2}=t, \quad t \in \mathbb{R}
$$

с произвольной постоянной $t_{0}$ получается траектория гамильтоновой системы. Параметризация неустойчивого (устойчивого) многообразия имеет ограниченное аналитическое продолжение в полуплоскость $\operatorname{Re} t_{1}<-\delta\left(\right.$ соответственно в $\left.\operatorname{Re} t_{1}>\delta\right)$ для некоторой постоянной $\delta>0$. Параметризация, удовлетворяющая перечисленным выше свойствам, единственна при условии $x^{u}(0,0 ; \varepsilon)=x_{0}(0)\left(x^{s}(0,0 ; \varepsilon)=x_{0}(0)\right)$.

Шаг 2. Выпрямление потока. Параметры $\left(t_{1}, t_{2}\right)$ можно рассмотреть как систему координат на соответствующем инвариантном многообразии, при этом ограничение потока на инвариантное многообразие в этих координатах имеет вид прямолинейного и равномерного движения. Предположим, что для некоторого $m>0$ эта система может быть продолжена до системы координат в $\varepsilon^{m}$-окрестности (в комплексифицированном фазовом пространстве) сегмента сепаратрисы, отвечающего $t_{1} \in D$, $\varepsilon^{-1} t_{2} \in \mathbb{S}^{1}$

$$
D=\left\{t_{1} \in \mathbb{C}:\left|\operatorname{Re} t_{1}\right| \leqslant 10 \varepsilon,\left|\operatorname{Im} t_{1}\right| \leqslant \rho-\varepsilon\right\} .
$$

Сформулируем последнее утверждение подробнее. Существует система канонических координат $(T, E, t)$, в которых уравнения движения принимают вид

$$
\frac{d T}{d t}=1, \quad \frac{d E}{d t}=0
$$

при этом замена $(x, y) \mapsto(T, E)$ аналитична в описанной выше окрестности, периодична по $t$ с периодом $2 \pi \varepsilon$ и удовлетворяет нормировочному условию

$$
\left(\begin{array}{c}
x^{u}\left(t_{1}, t_{2} ; \varepsilon\right) \\
y^{u}\left(t_{1}, t_{2} ; \varepsilon\right) \\
t_{2}
\end{array}\right) \mapsto\left(\begin{array}{c}
t_{1} \\
0 \\
t_{2}
\end{array}\right)
$$

После этой замены координат

$$
\left(\begin{array}{c}
x^{s}\left(t_{1}, t_{2} ; \varepsilon\right) \\
y^{s}\left(t_{1}, t_{2} ; \varepsilon\right) \\
t_{2}
\end{array}\right) \mapsto\left(\begin{array}{c}
t_{1}+\mu\left(t_{1}-t_{2} ; \varepsilon\right) \\
\Theta\left(t_{1}-t_{2} ; \varepsilon\right) \\
t_{2}
\end{array}\right)
$$

где

$$
\begin{aligned}
& \Theta\left(t_{0} ; \varepsilon\right) \equiv E\left(x^{s}\left(t+t_{0}, t\right), y^{s}\left(t+t_{0}, t\right), t / \varepsilon ; \varepsilon\right), \\
& \mu\left(t_{0} ; \varepsilon\right) \equiv T\left(x^{s}\left(t+t_{0}, t\right), y^{s}\left(t+t_{0}, t\right), t / \varepsilon ; \varepsilon\right)-\left(t+t_{0}\right)
\end{aligned}
$$


являются аналитическими функциями одной переменной с периодом $2 \pi \varepsilon$. Площадь луночки равна следующему интегралу:

$$
\mathscr{A}(\varepsilon)=\int_{t_{01}}^{t_{02}} \Theta\left(t_{0} ; \varepsilon\right) d\left(t_{0}+\mu\left(t_{0} ; \varepsilon\right)\right),
$$

где пределы интегрирования соответствуют двум соседним нулям функции $\Theta$. Экспоненциально тонкие оценки для этой функции могут быть найдены при исследовании аналитического продолжения этой функции с помощью леммы о коэффициентах Фурье. Из этой леммы следует, в частности, что если функция $\mu$ ограничена в достаточно широкой полосе, то ее производная экспоненциально мала на вешественной оси, следовательно, ею можно пренебречь при вычислении интеграла (5.9).

Несложно проверить, что

$$
\begin{aligned}
& \frac{\partial E}{\partial x}\left(x^{u}\left(t_{1}, t_{2} ; \varepsilon\right), y^{u}\left(t_{1}, t_{2} ; \varepsilon\right), t_{2} ; \varepsilon\right)=-\frac{\partial y^{u}}{\partial t_{1}}\left(t_{1}, t_{2} ; \varepsilon\right), \\
& \frac{\partial E}{\partial y}\left(x^{u}\left(t_{1}, t_{2} ; \varepsilon\right), y^{u}\left(t_{1}, t_{2} ; \varepsilon\right), t_{2} ; \varepsilon\right)=\frac{\partial x^{u}}{\partial t_{1}}\left(t_{1}, t_{2} ; \varepsilon\right) .
\end{aligned}
$$

Тогда, использовав ряды Тейлора первого порядка, получаем

$$
\Theta\left(t_{0} ; \varepsilon\right)=\operatorname{det}\left|\begin{array}{ll}
\frac{\partial x^{u}}{\partial t_{1}} & x^{s}-x^{u} \\
\frac{\partial y^{u}}{\partial t_{1}} & y^{s}-y^{u}
\end{array}\right|\left(t+t_{0}, t\right)+O_{2},
$$

где $\mathrm{O}_{2}$ обозначает квадратичные по $x^{s}-x^{u}$ члены, а $t$ произвольно.

Шаг 3. Вывод асимптотических рядов для сепаратрис. Можно проверить, что существует ряд вида

$$
x^{u, s}\left(t_{1}, t_{2} ; \varepsilon\right) \sim \sum_{n \geqslant 0} \frac{\varepsilon^{n}}{n !} x_{n}\left(t_{1}, t_{2}\right),
$$

асимптотический как для устойчивой, так и для неустойчивой сепаратрисы. Первьй член этого ряда $x_{0}\left(t_{1}, t_{2}\right) \equiv x_{0}\left(t_{1}\right)$ соответствует сепаратрисе усредненной системы. Коэффищиенты $x_{n}$ не зависят от $\varepsilon$, имеют период по $t_{2}$, равньй $2 \pi \varepsilon$, ограничены в полосе $\left|\operatorname{Im} t_{1}\right|<\rho-\delta$ при любом $\delta>0$ и имеют особенности при $t_{1}= \pm i \rho$.

Соответственно разность $x^{s}-x^{u}$ оказьвается меньше любой степени $\varepsilon$ в той части комплексного фазового пространства, где этот ряд является асимптотикой для обеих сепаратрис. В частности, это верно на подмножестве $D$, определяемом неравенствами

$$
\left|\operatorname{Re} t_{1}\right| \leqslant 10 \varepsilon, \quad\left|\operatorname{Im} t_{1}\right| \leqslant \rho-\delta \text { при любом } \delta>0 \text {. }
$$

В частности, это означает, что область определения $\Theta\left(t_{0}\right)$ включает полосу $\left|\operatorname{Im} t_{0}\right|<$ $\rho-\delta$.

Этих рассуждений достаточно для доказательства того, что сепаратрисы совпадают с точностью до величины $O\left(\exp \left(-\frac{\rho-\delta}{\varepsilon}\right)\right)$. Нас же интересует оценка снизу для расщепления. Поэтому мы исследуем сепаратрисы при $\operatorname{Im} t_{1}-\rho=O\left(\varepsilon \ln \varepsilon^{-1}\right)$. 
Оказывается, что при этих значениях параметра расщепление уже не является экспоненциально малым по $\varepsilon$ и может быть обнаружено в конечном порядке теории возмушений.

Шаг 4. Построение модельной системы. Для исследования сепаратрис в окрестности особенности аппроксимации, полученной на третьем шаге, удобно сделать замену параметров и времени:

$$
s_{1}=\frac{t_{1}-i \rho}{\varepsilon}, \quad s_{2}=\frac{t_{2}}{\varepsilon}, \quad s=\frac{t}{\varepsilon} .
$$

Будем искать теперь гамильтонову систему, которую мы будем назьвать модельной и которая обладает инвариантными многообразиями (не обязательно связанными с периодической траекторией), которые апроксимируют сепаратрисы исходной системы в некоторой части фазового пространства.

Для исследования возмущений маятника удобно сделать замену

$$
u=i x-\ln \frac{2}{\varepsilon^{2}}, \quad v=i \varepsilon y .
$$

Эта замена, хотя и не является канонической, сохраняет гамильтонову форму уравнений движения, при этом новый гамильтониан имеет вид

$$
\begin{aligned}
& \widetilde{H}=\frac{v^{2}}{2}-e^{u-i a \sin s}-\frac{\varepsilon^{4}}{4} e^{-u+i a \sin s} \\
& \widetilde{H}=\frac{v^{2}}{2}-e^{u}(1-i a \cos s)-\frac{\varepsilon^{4}}{4} e^{-u}(1+i a \cos s)
\end{aligned}
$$

соответственно. При $\varepsilon=0$ получается модельная система. Она обладает двумя инвариантными многообразиями, представимьми с помошью функций

$$
\left(u_{+}\left(s_{1}, s_{2}\right), v_{+}\left(s_{1}, s_{2}\right)\right) \text { и }\left(u_{-}\left(s_{1}, s_{2}\right), v_{-}\left(s_{1}, s_{2}\right)\right) .
$$

Эти многообразия близки к исходным сепаратрисам, кроме того, имеет место оценка

$$
\operatorname{det}\left|\begin{array}{ll}
\frac{\partial x^{u}}{\partial t_{1}} & x^{s}-x^{u} \\
\frac{\partial y^{u}}{\partial t_{1}} & y^{s}-y^{u}
\end{array}\right|\left(t_{1}, t_{2}\right)=-\varepsilon^{-2} \operatorname{det}\left|\begin{array}{ll}
\frac{\partial u_{-}}{\partial s_{1}} & u_{+}-u_{-} \\
\frac{\partial v_{-}}{\partial s_{1}} & v_{+}-v_{-}
\end{array}\right|\left(s_{1}, s_{2}\right)(1+O(\varepsilon))
$$

справедливая на пересечении линии $\operatorname{Im} t_{1}=\rho-\sigma \varepsilon \ln \varepsilon^{-1}$ с областью $D$ при некотором положительном $\sigma>1$. Множитель $-\varepsilon^{-2}$ появляется из-за замен (5.11) и (5.12). Можно показать, что детерминант, стояший в правой части (5.13), близок к гармонической функции $\Theta_{1} \exp \left(-i\left(s_{1}-s_{2}\right)\right)$ (см. лемму 5.5.2). С учетом этого из (5.10) получаем

$$
\Theta\left(t_{0} ; \varepsilon\right)=-\varepsilon^{-2} \Theta_{1} \exp \frac{-\rho-i t_{0}}{\varepsilon}(1+O(\varepsilon))
$$


на отрезке прямой $\operatorname{Im} t_{0}=\rho-\sigma \varepsilon \ln \varepsilon^{-1}$. Функция $\Theta$ вешественно-аналитична, поэтому ее аппроксимация на комплексно-сопряженной линии получается автоматически. Таким образом,

$$
\begin{aligned}
\Theta\left(t_{0} ; \varepsilon\right) & =-\varepsilon^{-2} \Theta_{1} \exp \frac{-\rho-i t_{0}}{\varepsilon}(1+O(\varepsilon))-\varepsilon^{-2} \bar{\Theta}_{1} \exp \frac{-\rho+i t_{0}}{\varepsilon}(1+O(\varepsilon)) \\
& =-2 \varepsilon^{-2}\left|\Theta_{1}\right| e^{-\rho / \varepsilon} \cos \left(\frac{t_{0}}{\varepsilon}-\arg \Theta_{1}\right)+(\text { членш старших порядков })
\end{aligned}
$$

в полосе $\left|\operatorname{Im} t_{0}\right| \leqslant \rho-\sigma \varepsilon \ln \varepsilon^{-1}$. Вообще говоря, среднее значение $\Theta$ отлично от нуля, однако, используя тот факт, что плошадь, определяемая интегралом (5.9), при $t_{02}=t_{01}+2 \pi \varepsilon$ равна нулю, несложно проверить, что среднее значение экспоненциально мало по сравнению с погрешностью следуюшей формулы. Отметим, что соответствующие рассуждения работы [15] не верны и ведут к неправильному вьводу о равенстве среднего нулю. Применяя лемму о коэффициентах Фурье, получаем, что при вещественных $t_{0}$ члены более высокого порядка не превосходят $O\left(\varepsilon^{-1} e^{-\rho / \varepsilon}\right)$ :

$$
\Theta\left(t_{0} ; \varepsilon\right)=-2 \varepsilon^{-2}\left|\Theta_{1}\right| e^{-\rho / \varepsilon} \cos \left(\frac{t_{0}}{\varepsilon}-\arg \Theta_{1}\right)+O\left(\varepsilon^{-1} e^{-\rho / \varepsilon}\right)
$$

Эта аппроксимащия может быть использована для вычисления других характеристик расщепления, таких, например, как площадь луночки.

Проблема обоснования метода. Достаточно ясно, что не все из описанных выше шагов могут быть с равной легкостью реализованы для произвольной системы. Существование параметризации, описанной на первом шаге, является общим фактом и может быть вьведено как непосредственно, так и с помошью нормальных форм Биркгофа. Шаг 2 может быть оправдан с помошњю соответствующего метода для систем с двумя характерньми масштабами времени. Построение координатной системы $(T, E)$ второго шага является непростой и самой нестандартной частью доказательства.

Шаг 4 обладает наименьшей обшностью. Подстановка (5.12) пригодна для нахождения модельной системы для гамильтонианов, являющихся тригонометрическими полиномами по переменной $x$, при условии, что степень полинома в явно зависяшей от времени части не слишком велика по сравнению со степенью усредненной части.

5.5. Корректирующий множитель. Оставшаяся часть настоящей раздела посвяшена исследованию расшепления сепаратрис модельного гамильтониана

$$
\frac{v^{2}}{2}-\omega^{2}(1+g(s)) e^{u}
$$

где $\omega$ является некоторой постоянной, а функция $g$ является периодической с периодом $2 \pi$ и нулевым средним за период. Будем обозначать время переменной $s$. Функция $g$ подразумевается аналитической в полосе, содержащей вещественную ось. Последнее ограничение не носит принципиального характера, однако заметно упрощает выкладки, так как избавляет от необходимости контролировать производные в силу оценок Коши. 
Уравнения движения удобно рассматривать как автономную систему в расширенном фазовом пространстве:

$$
\begin{aligned}
& u^{\prime}=v, \\
& v^{\prime}=\omega^{2}(1+g(s)) e^{u}, \\
& s^{\prime}=1 .
\end{aligned}
$$

Проведем исследование двумерных инвариантных многообразий этой системы. Удобно искать их в параметрическом виде, выбрав параметры таким образом, чтобы траектории системы соответствовали

$$
s_{1}=s+s_{0}, \quad s_{2}=s, \quad s \in \mathbb{R}
$$

Будем искать $2 \pi$-периодическую по $s_{2}$ параметризацию. Отметим, что система уравнений эквивалентна одному уравнению второго порядка

$$
u^{\prime \prime}=\omega^{2}(1+g(s)) e^{u} .
$$

Формальное многообразие. Будем искать инвариантное многообразие уравнения (5.15) в виде

$$
u\left(s_{1}, s_{2}\right) \sim-\ln \frac{\omega^{2} s_{1}^{2}}{2}+\sum_{n \geqslant 2} \frac{a_{n}\left(s_{2}\right)}{n ! s_{1}^{n}},
$$

где функции $a_{k}$ являются $2 \pi$-периодическими. Так как $u\left(s+s_{0}, s\right)$ является решением уравнения (5.15) при произвольной комплексной постоянной $s_{0}$, получаем из $(5.15)$

$$
\frac{\partial^{2} u}{\partial s_{1}^{2}}\left(s_{1}, s_{2}\right)+2 \frac{\partial^{2} u}{\partial s_{1} \partial s_{2}}\left(s_{1}, s_{2}\right)+\frac{\partial^{2} u}{\partial s_{2}^{2}}\left(s_{1}, s_{2}\right)=\omega^{2}\left(1+g\left(s_{2}\right)\right) \cdot \exp u\left(s_{1}, s_{2}\right) .
$$

ЛЕмма 5.5.1. Существует и единственно формальное решение уравнения (5.17), имеющее вид (5.16).

ДокАЗАТЕЛЬСТво. Подстановка ряда (5.16) в уравнение (5.17) и последующая группировка членов одного порядка по $s_{1}^{-1}$ приводит к системе

$$
\begin{aligned}
2+\frac{a_{2}^{\prime \prime}}{2 !} & =2(1+g), \\
-\frac{2 a_{2}^{\prime}}{1 !}+\frac{a_{3}^{\prime \prime}}{3 !} & =0, \\
\frac{(n-1) a_{n-2}}{(n-3) !}-\frac{2 a_{n-1}^{\prime}}{(n-2) !}+\frac{a_{n}^{\prime \prime}}{n !} & =\frac{2(1+g) \mathscr{Y}_{n-2}\left(a_{1}, \ldots, a_{n-2}\right)}{(n-2) !}, \quad n \geqslant 4 .
\end{aligned}
$$

Здесь $\mathscr{Y}_{n}$ обозначают полиномы Белла [71], определяемые последовательно

$$
\begin{aligned}
\mathscr{Y}_{0} & =1 \\
\mathscr{Y}_{n}\left(a_{1}, \ldots, a_{n}\right) & =\sum_{k=0}^{n-1} C_{n-1}^{k} \mathscr{Y}_{k}\left(a_{1}, \ldots, a_{k}\right) a_{n-k}, \quad n \geqslant 1 .
\end{aligned}
$$


Эти полиномы позволяют построить переразложение экспоненты

$$
\exp \left(\sum_{k \geqslant 1} \frac{a_{k} z^{k}}{k !}\right)=\sum_{k \geqslant 0} \frac{z^{k}}{k !} \mathscr{Y}_{k}\left(a_{1}, \ldots, a_{k}\right) .
$$

$\mathrm{B}$ нашем случае $a_{1} \equiv 0$. Коэффициенты $a_{2}$ и $a_{3}$ могут быть определены из уравнений (5.18) и (5.19) с точностью до прибавления постоянной. Продолжим далее по индукции. Пусть $a_{1}, a_{2}, \ldots, a_{n-3}$ определены однозначно, а $a_{n-2}$ и $a_{n-1}$ определены с точностью до прибавления постоянной. Уравнение (5.20) может быть рассмотрено как уравнение на $a_{n}^{\prime \prime}$. Его решение может быть найдено с помощью разложения в ряды Фурье. Обозначая преобразование Фурье функции $а$ через $\mathscr{F}[a]$, имеем

$$
\begin{gathered}
(n-1)(n-2) \mathscr{F}\left[a_{n-2}\right](k)-2 i k \mathscr{F}\left[a_{n-1}\right](k)-\frac{k^{2}}{n(n-1)} \mathscr{F}\left[a_{n}\right](k) \\
=2\left(\mathscr{F}[1+g] * \mathscr{F}\left[\mathscr{Y}_{n-2}\right]\right)(k)
\end{gathered}
$$

для $k \in \mathbb{Z}$. Здесь “*” обозначает свертку. Это уравнение позволяет определить все коэффициенты $a_{n}$ за исключением нулевого. Правая часть зависит от коэффициента $a_{n-2}$, который входит в уравнение через $\mathscr{Y}_{n-2}$. Соответствуюшее слагаемое имеет неопределенньй нулевой коэффициент ряда Фурье. Рассмотрим это уравнение подробнее. Пусть в $(5.22) k=0$, получаем

$$
(n-1)(n-2) \mathscr{F}\left[a_{n-2}\right](0)=2\left(\mathscr{F}[1+g] * \mathscr{F}\left[\mathscr{Y}_{n-2}\right]\right)(0)
$$

Принимая во внимание (5.21), можно выписать явно зависимость правой части от $a_{n-2}$ :

$$
\begin{aligned}
(n-1)(n-2) \mathscr{F}\left[a_{n-2}\right](0)= & 2 \mathscr{F}\left[a_{n-2}\right](0)+2\left(\mathscr{F}[g] * \mathscr{F}\left[a_{n-2}\right]\right)(0) \\
& +2\left(\mathscr{F}[1+g] * \sum_{l=2}^{n-3} C_{n-3}^{l}\left(\mathscr{F}\left[\mathscr{Y}_{l}\right] * \mathscr{F}\left[a_{n-2-l}\right]\right)\right)(0)
\end{aligned}
$$

сумма начинается с $l=2$ в силу того, что $\mathscr{Y}_{1}=a_{1}=0$. Таким образом, имеет место равенство

$$
\begin{aligned}
\left(n^{2}-3 n\right) \cdot \mathscr{F}\left[a_{n-2}\right](0)= & 2\left(\mathscr{F}[g] * \mathscr{F}\left[a_{n-2}\right]\right)(0) \\
& +2\left(\mathscr{F}[1+g] * \sum_{l=2}^{n-3} C_{n-3}^{l}\left(\mathscr{F}\left[\mathscr{Y}_{l}\right] * \mathscr{F}\left[a_{n-2-l}\right]\right)\right)(0),
\end{aligned}
$$

при этом правая часть не зависит от нулевого коэффициента Фурье $a_{n-2}$. Эта формула может быть использована для нахождения этого коэффициента. После этого уравнение (5.22) определяет $a_{n}$ с точностью до постоянной. Таким образом, уравнение (5.20) определяет $a_{n}$ с точностью до постоянной, а постоянная в $a_{n-2}$ однозначно определяется из условия разрешимости этого уравнения в классе периодических функций. Принцип индукции влечет за собой утверждение леммы. 
Аналитическое многообразие. Вообще говоря, следует ожидать, что построенные ряды расходятся. Однако сушествуют аналитические решения уравнения, для которых построенное выше формальное решение является асимптотикой в секторах $\delta<\arg s_{1}<2 \pi-\delta$ и $-\pi+\delta<\arg s_{1}<\pi-\delta$ соответственно.

Доказательство этого факта аналогично проведенному ранее в [48] при исследовании полустандартного отображения. Основная идея состоит в поиске решения как суммы конечного отрезка ряда и малой неизвестной функции. Подстановка в уравнение движения позволяет построить дифференщиальное уравнение на новую неизвестную функцию. После этого необходимо выделить линейный дифференциальный оператор второго порядка, обрашение которого приводит к интегральному уравнению, которое может быть решено методом итераций. При этом есть два способа обращения дифференщиального оператора, один из них соответствует фиксации асимптотического поведения на плюс бесконечности, а другой - на минус бесконечности.

Обозначая первую компоненту соответствуюших параметризаций через $u_{-}\left(s_{1}, s_{2}\right)$ и $u_{+}\left(s_{1}, s_{2}\right)$, получаем при любом $N \geqslant 0$

$$
u_{+}\left(s_{1}, s_{2}\right)-u_{-}\left(s_{1}, s_{2}\right)=o\left(s_{1}^{N}\right)
$$

в каждом секторе вида $-\pi+\delta<\arg s_{1}<-\delta$. В действительности эти многообразия экспоненщиально близки. Как и при исследовании полустандартного отображения, можно доказать, что

$$
\begin{gathered}
\left|u_{+}\left(s_{1}, s_{2}\right)-u_{-}\left(s_{1}, s_{2}\right)\right| \leqslant \text { const } \cdot\left|s_{1}\right|^{2} \exp \left(-\left|\operatorname{Im} s_{1}\right|\right), \\
\left|v_{+}\left(s_{1}, s_{2}\right)-v_{-}\left(s_{1}, s_{2}\right)\right| \leqslant \text { const } \cdot\left|s_{1}\right| \exp \left(-\left|\operatorname{Im} s_{1}\right|\right)
\end{gathered}
$$

при условии $\operatorname{Im} s_{1} \leqslant-A$ в секторе $-\pi+\delta<\arg s_{1}<-\delta$. Постоянная в оценке зависит от выбора $A$ и $\delta$.

Оценка расстояния между сепаратрисами модельной системы. Определитель

$$
\Psi\left(s_{1}, s_{2}\right)=\operatorname{det}\left|\begin{array}{ll}
\frac{\partial u_{-}}{\partial s_{1}} & u_{+}-u_{-} \\
\frac{\partial v_{-}}{\partial s_{1}} & v_{+}-v_{-}
\end{array}\right|\left(s_{1}, s_{2}\right)
$$

является удобной величиной, характеризующей величину расшепления.

Лемма 5.5.2. Существует комплексная постоянная $\Theta_{1}$ такая, что в секmope $-\pi+\delta<\arg s_{1}<-\delta$

$$
\Psi\left(s_{1}, s_{2}\right)=\Theta_{1} \exp \left(-i\left(s_{1}-s_{2}\right)\right)+O\left(\exp \left(-\left(2-\delta^{\prime}\right)\left|\operatorname{Im} s_{1}\right|\right)\right)
$$

при произвольных положительных постоянных $\delta^{\prime}, A\left(\operatorname{Im} s_{1}<-A\right)$. Постоянные в оченке погрешности зависят от $A, \delta$ и $\delta^{\prime}$.

Если функция $g$ и постоянная $\omega$ зависят аналитически от параметра $a$, определенного на некотором компактном подмножестве комплексной плоскости, и на этом подмножестве $\omega$ отделена от нуля постоянной, то $\Theta_{1}$ и функции $u_{ \pm}$также зависят аналитически от $a$. 
Корректирующий множитель. Пусть $g(s ; a)$ аналитична по $a$ в окрестности нуля при всех $s$, кроме того, пусть $g(s ; 0) \equiv 0$. Тогда для гамильтониана $\frac{v^{2}}{2}-(1+g(s ; a)) e^{u}$ имеет место

$$
\Theta_{1}(a)=-4 \pi i a \frac{d g_{1}}{d a}(0) \cdot(1+O(a))
$$

где $g_{1}(a)$ является первым членом в разложении функции $g$ в ряд $\Phi$ урье: $g(s, a)=$ $\sum_{k=-\infty}^{\infty} g_{k}(a) e^{i k s}$. При малых $a$ для нахождения постоянной $\Theta_{1}(a)$ можно применить метод Мельникова:

$$
\Theta_{1}(a)=-4 \pi i a g_{1}^{\prime}(0)+O\left(a^{2}\right) .
$$

Корректируюший множитель - это функция, определенная равенством

$$
f(a)=\frac{\Theta_{1}(a)}{-4 \pi i a g_{1}^{\prime}(0)} .
$$

Таким образом, корректируюший множитель описьвает отклонение истинной асимптотики от величины, предсказьваемой с помощью метода Мельникова.

\section{6. Заключение}

В настоящее время центр внимания исследователей перемешается в сторону систем с тремя и более степенями свободы. Это связано в первую очередь с тем, что расщепление сепаратрис является ключевым элементом возникновения глобальной неустойчивости, назьваемой также диффузией Арнольда. Несмотря на то, что со времени открытия механизма неустойчивости прошло уже более 30 лет и нет сомнений в том, что подобное явление имеет место в системах общего положения, доказательств наличия диффузии Арнольда для конкретных аналитических систем в настоящее время почти не имеется. Исключение составляют некоторые системы, носящие несколько искусственньй характер. Такое положение возникло благодаря ряду существенных трудностей, одной из которых является экспоненциальная малость расшепления сепаратрис. Упомянем несколько недавних работ, в которых сделаны первые шаги в исследовании расщепления сепаратрис для многомерных гамильтоновых систем, близких к интегрируемым: [17], [82], [25], [26], [60].

Другим любопытным направлением является исследование возможности применения разработанных методов к задачам, непосредственно возникаюшим из механики или теоретической физики [42]-[44].

Отметим в заключение некоторые работы, посвященные задачам об экспоненциально малом расщеплении сепаратрис в негамильтоновой постановке: [2], [61].

\section{СПИСОК ЛИТЕРАТУРЫ}

[1] В. М. Алексеев. Квазислучайные динамические системы. I-III // Матем. сб. 1968. Т. 76. № 1. C. 72-134; 1968. T. 77. № 4. C. 545-601; 1969. T. 78. № 1. C. 3-50.

[2] C. Amick, E.S.C. Ching, L.P. Kadanoff, V. Rom-Kedar. Beyond all orders: singular perturbations in a mapping // J. Nonlinear Sci. 1992. V. 2. P. 9-67.

[3] S. Angenent. A variational interpretation of Melnikov's function and exponentially small separatrix splitting // London Math. Soc. Lecture Note Ser. 1993. V. 192. P. 5-35.

[4] В.И. Арнольд. О неустойчивости в гамильтоновых системах со многими степенями свободы // Докл. АН СССР. 1964. Т. 156. №1. С. 9-12. 
[5] В.И. Арнольд, В.В. Козлов, А. И. Нейштадт. Математические аспекты классической и небесной механики // Итоги науки и техники. Совр. пробл. матем. Фундаментальные направления. Т. 3. М.: ВИНИТИ, 1985.

[6] A. Benseny, C. Olivé. High precision angles between invariant manifolds for rapidly forced Hamiltonian systems // International Conference on Differential Equations (Equa $\left.\frac{\partial i}{\partial t} f f 91\right)$, Barcelona, 1991. Singapore: World Scientific, 1993. P. 315-319.

[7] Н.Н. Боголюбов, Ю. А. Митропольский. Асимптотические методы в теории нелинейных колебаний. М.: Наука, 1974.

[8] Y.H. Chang. Proof of an asymptotic symmetry of the rapidly forced pendulum // NATO Adv. Sci. Inst. Ser. B Phys. 1991. V. 284. P. 213-221.

[9] Y.H. Chang, H. Segur. An asymptotic symmetry of the rapidly forced pendulum // Phys. D. 1991. V. 51. № 1-3. P. 109-118.

[10] V. L. Chernov. On separatrix splitting of some quadratic area-preserving maps of the plane // Regul. Chaotic Dynam. 1998. V. 3. № 1. P. 49-65.

[11] V. L. Chernov. Separatrix splitting for the Henón map: a resurgence approach // Preprint № 188 (Math. Preprint Ser.). Barcelona: Universitat de Barcelona, 1995.

[12] B. V. Chirikov. A universal instability of many-dimensional oscillator systems // Phys. Rep. 1979. V. 52. P. 263-379.

[13] Б. В. Чириков. Аномалњная диффузия в микротроне и критическая структура на границе хаоса // ЖЭТФ. 1996. Т. 110. № 4. С. 1174-1185.

[14] R. Cushman. Examples of nonintegrable analytic Hamiltonian vector fields with no small divisors // Trans. Amer. Math. Soc. 1978. V. 238. № 1. P. 45-55.

[15] A. Delshams, T.M. Seara. An asymptotic expression for the splitting of separatrices of the rapidly force pendulum // Comm. Math. Phys. 1992. V. 150. P. 433-463.

[16] A. Delshams, T.M. Seara. Splitting of separatrices in Hamiltonian systems with one and a half degrees of freedom // Math. Phys. Electron. J. 1997. V. 3. №4. P. 1-40.

[17] A. Delshams, V. Gelfreich, À. Jorba, T. M. Seara. Exponentially small splitting of separatrices under fast quasiperiodic forcing // Comm. Math. Phys. 1997. V. 189. № 1 . P. 35-71.

[18] R.L. Devaney. Transversal homoclinic orbit in an integrable system // Amer. J. Math. 1978. V. 100. № 3. P. 631-642.

[19] J. A. Ellison, M. Kummer, A. W. Sáenz. Transcendentally small transversality in the rapidly forced pendulum // J. Dynam. Differential Equations. 1993. V. 5. № 2. P. 241-277.

[20] B. Fiedler, J. Scheurle. Discretization of Homoclinic Orbits, Rapid Forcing and "Invisible" Chaos. Providence, R.I.: Amer. Math. Soc., 1996. (Mem. Amer. Math. Soc. V. 570.)

[21] Н. Н. Филоненко, Р. З. Сагдеев, Г. М. Заславский. Distribution of magnetic surfaces by magnetic field irregularity. II // Nuclear Fusion. 1967. V. 7. P. 253-266.

[22] E. Fontich. Rapidly forced planar vector fields and splitting of separatrices // J. Differential Equations. 1995. V. 119. № 2. P. 310-335.

[23] E. Fontich, C. Simó. The splitting of separatrices for analy tic diffeomorphisms // Ergodic Theory Dynam. Systems. 1990. V. 10. P. 295-318.

[24] G. Gallavotti. Twistless KAM tori, quasi flat homoclinic intersections, and other cancellations in the perturbation series of certain completely integrable Hamiltonian systems. A review // Rev. Math. Phys. 1994. V. 6. № 3. P. 343-411.

[25] G. Gallavotti, G. Gentile, V. Mastropietro. Separatrix splitting for systems with three time scales // Comm. Math. Phys. 1999. V. 202. P. 197-236.

[26] G. Gallavotti, G. Gentile, V. Mastropietro. On homoclinic splitting problems // Phys. D. 2000. V. 137. P. 202-204.

[27] V. G. Gelfreich. Separatrices splitting for the rapidly forced pendulum // Seminar on Dynamical Systems. St. Petersburg, 1991. (Progr. Nonlinear Differential Equations Appl. V. 12.) / ed. S. Kuksin, V.F. Lazutkin, J. Pöschel. Basel: Birkhäuser, 1994. P. 47-67.

[28] V.G. Gelfreich. Conjugation to a shift and the splitting of invariant manifolds // Appl. Math. 1996. V. 24. № 2. P. 127-140. 
[29] V. G. Gelfreich. Separatrix splitting for a high-frequency perturbation of the pendulum // Russian J. Math. Phys. 2000. V. 7. № 1. P. 48-71.

[30] V.G. Gelfreich. Melnikov method and exponentially small splitting of separatrices // Phys. D. 1997. V. 101. P. 227-248.

[31] V.G. Gelfreich. Reference systems for splitting of separatrices // Nonlinearity. 1997. V. 10. P. $175-193$.

[32] V.G. Gelfreich. A proof of the exponentially small transversality of the separatrices for the standard map // Comm. Math. Phys. 1999. V. 201. № 1. P. 155-216.

[33] V. G. Gelfreich. Splitting of a small separatrix loop near the saddle-center bifurcation in area-preserving maps // Phys. D. 2000. V. 136. P. 266-279.

[34] В. Г. Гельфрейх. Расщепление сепаратрис стандартного трехпараметрического семейства отображений // Деп. в ВИНИТИ 3245-V88, 1988.

[35] В. Г. Гельфрейх. Расщепление сепаратрис полиномиальных отображений, сохраняющих площадь // Проблемы матем. физ. (Изд-во Ленинград. ун-та). 1991. № 13. С. 108-116.

[36] V. G. Gelfreich, D. Sauzin. Borel summation and splitting of separatrices for the Hénon map // Notes Scientifiques et Techniques du Bureau des Longitudes. 1999. № S067.

[37] V.G. Gelfreich, V.F. Lazutkin, N. V. Svanidze. A refined formula for the separatrix splitting for the standard map // Phys. D. 1994. V. 71. № 2. P. 82-101.

[38] V.G. Gelfreich, V.F. Lazutkin, M.B. Tabanov. Exponentially small splitting in Hamiltonian systems // Chaos. 1991. V. 1. № 2. P. 137-142.

[39] V. G. Gelfreich, V.F. Lazutkin, C. Simó, M. B. Tabanov. Fern like structures in the wild set of the standard and semistandard maps in $\mathbf{C}^{2} / /$ Internat. J. Bifur. Chaos Appl. Sci. Engrg. 1992. V. 2. № 2. P. 353-373.

[40] V. Hakim, K. Mellick. Exponentially small splittings of separatrices, matching in the complex plain and Borel summation // Nonlinearity. 1993. V. 6. P. 57-70.

[41] P. Holmes, J. Marsden, J. Scheurle. Exponentially small splitting of separatrices with applications to KAM theory and degenerate bifurcations // Contemp. Math. 1988. V. 81. P. 213-244.

[42] A. V. Ivanov. Study of the double mathematical pendulum. I: Numerical investigation of homoclinic transversal intersections // Regul. Chaotic Dynam. 1999. V. 4. № 1. P. 104-116.

[43] A. V. Ivanov. Study of the double mathematical pendulum. II: Investigation of exponentially small homoclinic intersections // Preprint № 271 (Math. Preprint Ser.). Barcelona: Universitat de Barcelona, 1999.

[44] A. V. Ivanov. Study of the double mathematical pendulum. III: Melnikov's method applied to the system in the limit of small ratio of pendulums masses // Reg. Chaotic Dynam. 2000. V. 5. № 3. P. 329-344.

[45] В.В. Козлов. Интегрируемость и неинтегрируемость в гамильтоновой механике // УМН. 1983. Т. 38. №1. С. 3-67.

[46] В. В. Козлов. Симметрии, топология и резонансы в гамильтоновой механике. Ижевск: Изд-во Удмурт. гос. ун-та, 1995.

[47] M. Kummer, J.A. Ellison, A.W. Sáenz. Exponentially small phenomena in the rapidly forced pendulum // NATO Adv. Sci. Inst. Ser. B Phys. 1991. V. 284. P. 197-211.

[48] V.F. Lazutkin. Exponential splitting of separatrices and an analytical integral for the semistandard map // Preprint. Paris: Université Paris VII, 1991.

[49] V.F. Lazutkin. Resurgent approach to the separatrices splitting // International Conference on Differential Equations (Equa $\frac{\partial i}{\partial t} f f 91$ ), Barcelona, 1991. Singapore: World Scientific, 1993. P. 163-176.

[50] V. F. Lazutkin. KAM Theory and Semiclassical Approximations to Eigenfunctions. Berlin: Springer-Verlag, 1993.

[51] В.Ф. Лазуткин. К теореме Мозера об инвариантных кривых // Вопросы динамической теории распространения сейсмических волн (Изд-во Ленинград. ун-та). 1974. № 14. C. $109-120$.

[52] В.Ф. Лазуткин. Расщепление сепаратрис для стандартного отображения Чирикова // Деп. в ВИНИТИ 6372/84, 1984. 
[53] В.Ф. Лазуткин. Расщепление сепаратрис для стандартного семейства преобразований, сохраняющих площадь // Проблемы матем. физ. (Изд-во Ленинград. ун-та). 1986. №12. C. $32-41$.

[54] В.Ф. Лазуткин. Расщепление комплексных сепаратрис // Функц. анализ и его прил. 1988. T. 22. № 2. C. 83-84.

[55] В.Ф. Лазуткин. О ширине зоны неустойчивости около сепаратрисы стандартного отображения // Докл. РАН. 1990. Т. 313. № 2. С. 268-272.

[56] V.F. Lazutkin. An analytic integral along the separatrix of the semistandard map: existence and an exponential estimate for the distance between the stable and unstable separatrices // Алгебра и анализ. 1992. Т. 4. № 4. С. 110-142.

[57] V.F. Lazutkin, I. G. Schachmanski, M. B. Tabanov. Splitting of separatrices for standard and semistandard mappings // Phys. D. 1989. V. 40. P. 235-348.

[58] В.Ф. Лазуткин, М.Б. Табанов, И.Г. Шахманский. Расщепление сепаратрис стандартного и полустандартного отображений // Деп. в ВИНИТИ 4027/85, 1985.

[59] А. Лихтенберг, М. Либерман. Регулярная и стохастическая динамика. М.: Мир, 1984.

[60] P. Lochak, J.-P. Marco, D. Sauzin. On the splitting of invariant manifolds in multidimensional near-integrable Hamiltonian systems // Prepublication № 220. Paris: Institut de Mathématiques de Jussieu, 1999.

[61] E. Lombardi. Oscillatory integrals and phenomena beyond all algebraic orders with applications to homoclinic orbits in reversible systems // Preprint № 99.31. Nice: Institut Non Linéaire de Nice, 1999.

[62] E. M. McMillan. A problem in the stability of periodic systems // Topics in Modern Physics. Colorado Association University Press, 1971. P. 219-244.

[63] J. Moser. The analytical invariants of an area preserving mapping near a hyperbolic fixed point // Comm. Pure Appl. Math. 1956. V. 9. P. 673-692.

[64] А. И. Нейштадт. Оценки в теореме Колмогорова о сохранении условно-периодических движений // ПММ. 1981. Т. 45. №6. С. 1016-1025.

[65] А. И. Нейштадт. О разделении движений в системах с быстро вращающейся фазой // ПMМ. 1984. Т. 48. № 2. С. 197-204.

[66] A. Nikitin. Separatrix splitting via resurgence for the "twist map". A method of calculation of the coefficients of the splitting formula // Preprint № 184 (Math. Preprint Ser.). Barcelona: Universitat de Barcelona, 1995.

[67] 3. Нитецки. Введение в дифференциальную динамику. М.: Мир, 1975.

[68] Я. Б. Песин. Характеристические показатели Ляпунова и гладкая эргодическая теория // УМН. 1977. Т. 32. № 4. С. 55-111.

[69] А. Пуанкаре. Новые методы небесной механики. Избранные труды. Т. 1. М.: Наука, 1971.

[70] А. Пуанкаре. О проблеме трех тел и уравнениях динамики. Избранные труды. Т. 2. М.: Наука, 1972.

[71] ЖК. Риордан. Введение в комбинаторньй анализ. М.: ИЛ, 1984.

[72] R. C. Robinson. Generic properties of conservative systems. I; II // Amer. J. Math. 1970. V. 92. P. 562-603; 897-906.

[73] J. Sanders. Melnikov's method and averaging // Celestial Mech. 1982. V. 28. P. 171-181.

[74] D. Sauzin. Résurgence paramétrique et exponentielle petitesse de l'écart des séparatrices du pendule rapidement forcé // Ann. Inst. Fourier (Grenoble). 1995. V. 45. № 2. P. 453-511.

[75] J. Scheurle. Chaos in a rapidly forced pendulum equation // Contemp. Math. 1989. V. 97. P. 411-419.

[76] J. Scheurle, J. Marsden, P. Holmes. Exponentially small estimates for separatrix splittings // NATO Adv. Sci. Inst. Ser. B Phys. 1991. V. 284. P. 187-195.

[77] C. Siegel, J. Moser. Lectures on Celestial Mechanics. Berlin: Springer-Verlag, 1971.

[78] C. Simó, E. Fontich. On the smallness of the angle between split separatrices // Singularités, Feuilletages et Mécanique Hamiltonienne / ed. M. Hermann, 1985. P. 41-52. 
[79] Yu. B. Suris. On the complex separatrices of some standard-like maps // Nonlinearity. 1994. V. 7. № 4. P. 1225-1236.

[80] Ю. Б. Сурис. Об интегрируемых отображениях типа стандартного // Функц. анализ и его прил. 1989. Т. 23. № 1. С. $84-85$.

[81] Д. В. Трещев. Введение в теорию возмущений гамильтоновых систем. М.: Фазис, 1998.

[82] Д. В. Трещев. Расщепление сепаратрис с точки зрения симплектической геометрии // Матем. заметки. 1997. Т. 61. №6. С. 890-906.

[83] Д. В. Трешев. Метод непрерывного усреднения в задаче о разделении быстрых и медленных движений // Регулярная и хаотическая динамика. 1997. Т. 2. № 3/4. С. 9-20.

[84] D. V. Treshchev. An averaging method for Hamiltonian systems, exponentially close to integrable ones // Chaos. 1996. V. 6. № 1. P. 6-14.

[85] D. V. Treshchev. Separatrix splitting for a pendulum with rapidly oscillating suspension point // Russian J. Math. Phys. 1997. V. 5. № 1. P. 63-98.

[86] D. V. Treshchev. Width of stochastic layers in near-integrable two-dimensional symplectic maps // Phys. D. 1998. V. 116. P. 21-43.

[87] Sh. Ushiki. Sur les liaisons-cols des systèmes dynamiques analytiques // C. R. Acad. Sci. Paris Ser. A. 1980. V. 291. P. 447-449.

[88] В. В. Вечеславов, Б. В. Чириков. Прецезионное измерение расщепления сепаратрисы нелинейного резонанса // Препринт № 98-3. Новосибирск: ИЯФ, 1998.

[89] Г. М. Заславский, Н. Н. Филоненко. Стохастическая неустойчивость захваченных частиц и условия применимости квазилинейного приближения // ЖЭТФ. 1968. Т. 54. № 5. C. $1590-1602$.

[90] Г. М. Заславский, М. Ю. Захаров, Р. З. Сагдеев, Д. А. Усиков, А. А. Черников. Стохастическая паутина и диффузия частиц в магнитном поле // ЖЭТФ. 1986. T. 91 . № 2 (8). C. 500-516. 